MZ-TH/05-08

hep-ph/0506286

June 2005

\title{
On the evaluation of a certain class of Feynman diagrams in $x$-space: Sunrise-type topologies at any loop order
}

\author{
S. Groote ${ }^{1,2}$, J.G. Körner ${ }^{2}$ and A.A. Pivovarov ${ }^{2,3}$ \\ ${ }^{1}$ Tartu Ülikooli Teoreetilise Füüsika Instituut, \\ Tähe 4, EE-51010 Tartu, Estonia \\ ${ }^{2}$ Institut für Physik der Johannes-Gutenberg-Universität, \\ Staudinger Weg 7, D-55099 Mainz, Germany \\ ${ }^{3}$ Institute for Nuclear Research of the \\ Russian Academy of Sciences, Moscow 117312, Russia
}

\begin{abstract}
We review recently developed new powerful techniques to compute a class of Feynman diagrams at any loop order, known as sunrise-type diagrams. These sunrise-type topologies have many important applications in many different fields of physics and we believe it to be timely to discuss their evaluation from a unified point of view. The method is based on the analysis of the diagrams directly in configuration space which, in the case of the sunrise-type diagrams and diagrams related to them, leads to enormous simplifications as compared to the traditional evaluation of loops in momentum space. We present explicit formulae for their analytical evaluation for arbitrary mass configurations and arbitrary dimensions at any loop order. We discuss several limiting cases of their kinematical regimes which are e.g. relevant for applications in HQET and NRQCD. We completely solve the problem of renormalization using simple formulae for the counterterms within dimensional regularization. An important application is the computation of the multi-particle phase space in $D$-dimensional space-time which we discuss. We present some examples of their numerical evaluation in the general case of $D$-dimensional space-time as well as in integer dimensions $D=D_{0}$ for different values of dimensions including the most important practical cases $D_{0}=2,3,4$. Substantial simplifications occur for odd integer space-time dimensions where the final results can be expressed in closed form through elementary
\end{abstract}


functions. We discuss the use of recurrence relations naturally emerging in configuration space for the calculation of special series of integrals of the sunrise topology. We finally report on results for the computation of an extension of the basic sunrise topology, namely the spectacle topology and the topology with an irreducible loop addition.

PACS ???? 


\section{Contents}

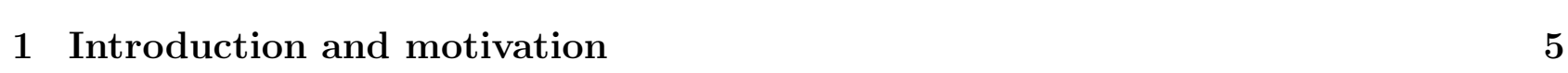

2 Basic notions and relations for sunrise-type diagrams 10

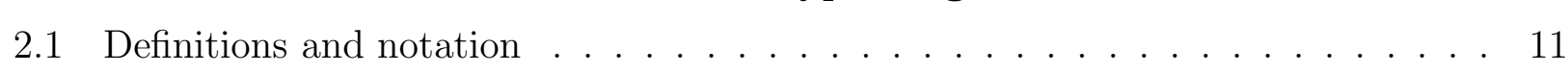

2.2 Momentum versus configuration space representation:Fourier transform in the evaluation of sunrise

2.3 Regularization and renormalization . . . . . . . . . . . . . 15

2.4 The spectrum as discontinuity across the physical cut . . . . . . . . . . . . . . . 17

3 "Tool box" for a practical analysis 20

3.1 Extraction of poles in dimensional regularization . . . . . . . . . . . . 20

3.2 Singular and finite parts for particular parameter values of the genuine sunrise: warm-up example

3.3 Three-loop sunrise-tvpe diagram for special momental . . . . . . . . . . . . 26

3.4 Infinite parts of sunrise-type diagrams with different masses at any number of loops: general techn

3.5 Finite part for the genuine sunrise with three different masses . . . . . . . . . . 28

3.6 Calculation of the finite part: further examples _. . . . . . . . . . . 29

3.7 Higher order expansion in $\varepsilon$ : general techniques and the four-loop vacuum bubble 30

$3.8 \varepsilon$-expansion of the five-loop vacuum bubble . . . . . . . . . . . . . . . . . 35

3.9 The spectral density, the discontinuity across the physical cut and the evaluation of the multi-part

4 Asymptotic analysis of sunrise-type diagrams $\quad 38$

4.1 Large momentum expansion: close to the massless limit . . . . . . . . . . . . . . 39

4.2 Small momentum expansion: close to the vacuum bubble . . . . . . . . . . . . . 40

4.3 Dominating mass expansion: near the static limit . . . . . . . . . . . . . . . 40

4.4 Threshold expansion: close to nonrelativistic phvsics . . . . . . . . . . . . . . . 41

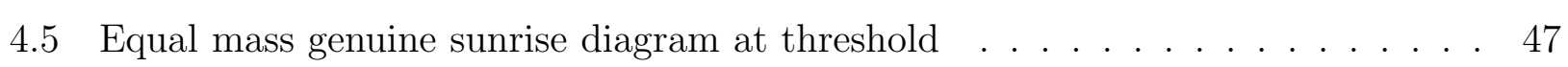

4.6 Three loops and a route to anv number of loops at threshold . . . . . . . . . . 48

4.7 New features of the threshold expansion: resummation of small mass effects for strongly asvmmetr

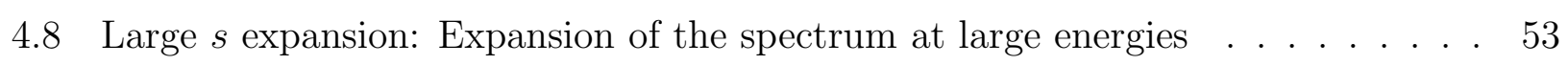

4.9 Large $s$ expansion for massive one- and multiloop diagrams . . . . . . . . . . . . 55

5 Non-standard propagators and other exotic settings 59

5.1 Odd-dimensional case . . . . . . . . . . . . . . . . . . . . . . 59

5.2 Large powers of propagators . . . . . . . . . . . . . . . . . 62

5.3 The example $B_{N}(0,0,2,2,2,2) \ldots \ldots \ldots$. . . . . . . . . . . . . 64 
5.4 The example $B_{N}(0.0 .2 .2 .2 .1) \ldots \ldots \ldots \ldots \ldots$. . . . . . . . . . . . 66

5.5 The example $B_{N}(0,0.2 .3,3,4) \ldots \ldots \ldots$

5.6 The reduction procedure . . . . . . . . . . . . . . . . . 67

5.7 Laplace-tvpe asvmptotics . . . . . . . . . . . . . . . . . . . . . . . . . . . 68

5.8 Irreducible numerator: three-loop vacuum bubble case . . . . . . . . . . . . . . . 69

5.9 Irreducible numerator: four-loop vacuum bubble case . . . . . . . . . . . . . . . 70

6 Generalization: Still within reach $\quad 72$

6.1 Generalization to the spectacle topology . . . . . . . . . . . . . . . . . 72

6.2 Example for the spectacle diagram . . . . . . . . . . . . . . . . . . . . . 74

6.3 About the occurrence of Clausen's dilogarithms . . . . . . . . . . . . . . . . . 75

6.4 The insertion of massless irreducible loops . . . . . . . . . . . . . . . . . . . . . 77

6.5 The insertion of massive irreducible loops . . . . . . . . . . . . . . . . . . . . . . 78

$\begin{array}{lll}7 & \text { Summary and conclusion } & 79\end{array}$

$\begin{array}{lr}\text { A Properties of Bessel functions } & 80\end{array}$

B Properties of Gegenbauer polynomials $\quad 82$

$\begin{array}{lr}\text { C Cuts and discontinuities } & 83\end{array}$

$\begin{array}{ll}\text { D Singular contributions up to four loops } & 86\end{array}$

\begin{tabular}{llr}
\hline E Subtraction terms for the small $x$ singularities & 87
\end{tabular}

$\begin{array}{lll}\text { F Analytical results for the four-loop sunrise diagram } & 87\end{array}$

$\begin{array}{lr}G \text { Integrand for the numerical integration } & 88\end{array}$ 


\section{Introduction and motivation}

In the present-day analysis of various physical processes the complexity of the problem often makes it impossible to find a complete mathematical description of the experimentally measured or observed quantities. In order to proceed one usually simplifies the mathematical model which describes the bulk of the phenomena. The more delicate effects are accounted for through an expansion in terms of a small parameter which has to be defined such that one retains full quantitative control in the analysis. The art of a physicist consists in splitting the whole analysis into a main part and into small corrections to the main part for which one develops a perturbative expansion. One may quote one of the greatest physicists of the last century, L.D. Landau, who used to say that physics starts when a small parameter of the problem has been found. The usefulness and power of such an approach has been proven over and over for many years. An example that comes into mind are the first accurate calculations analyzing high precision data from celestial mechanics in terms of Newton's law of gravitation. When observational data on the motion of the planets became more accurate it was no longer sufficient to take into account only their gravitational interaction with the sun but it was necessary also to include the small corrections arising from the mutual gravitational interactions between the planets themselves. This was done through perturbation theory since the full equations for the planetary movements with mutual interaction fully taken into account are too difficult to treat analytically even within standard classical mechanics. A remarkable fact is that these corrections are very small but still have to be taken into account since the data are extremely precise. This is well illustrated by a historical example taken from astronomy which was the most precise scientific discipline in the past: the planets of the solar system close to Earth were directly observed while an eighth planet - Neptune - was first introduced in order to explain slight perturbations in the orbit of Uranus. The orbit of Neptune was theoretically calculated with high precision and the planet was directly observed later at the predicted place.

Let us now turn to high energy physics. The main interest of fundamental research in high energy physics is to find new physical phenomena and to place them into a consistent logical picture of the natural world which mankind inhabits. It is generally believed that the structure of fundamental interactions (except gravity) is qualitatively understood at the energies available at present accelerators. Basically there are two trends for the search of new physics. One is aiming at a direct discovery of new physical phenomena and fundamental constituents of matter (elementary particles) by moving to higher and higher energies. This requires ever more powerful accelerating facilities while the precision of the experimental detection and theoretical 
calculations can remain at a moderate level. The other is an indirect search for new physics at low energies (mainly through radiative corrections) which is based on very precise experimental data at moderate energies and a high accuracy of the theoretical calculations [1, 2, 3, 4, 5]. A very recent example of the second category is the ongoing discussion about the possible appearance of deviations from the Standard Model (SM) of strong and electroweak interaction in new data on the muon anomalous magnetic moment [6, 7, 8, 9]. This two-fold road to scientific discovery is well known and has been pursued for ages as illustrated by the above example from celestial mechanics.

The muon anomalous magnetic moment has been computed in Quantum Electrodynamics (QED) - the most precise physical theory of the present time. QED is a highly accurate theory of electromagnetic particle interactions where predictions for physical observables are calculated within perturbation theory in the form of a series in the expansion parameter of the theory, the fine structure constant $\alpha$ with a numerical value of $\alpha^{-1}=137.036$ at zero momentum. The fact that $\alpha$ is rather small makes the expansions well convergent. The muon anomalous magnetic moment has been computed through the expansion in the small ratio of lepton masses and the expansion in the coupling constant up to the order $\alpha^{4}$. The most recent result reads [10, 11, 12]

$$
\alpha_{\mu}(\mathrm{QED})=A_{1}+A_{2}\left(m_{\mu} / m_{e}\right)+A_{2}\left(m_{\mu} / m_{\tau}\right)+A_{3}\left(m_{\mu} / m_{e}, m_{\mu} / m_{\tau}\right)
$$

where for instance [13]

$$
\begin{aligned}
A_{1}= & 0.5\left(\frac{\alpha}{\pi}\right)-0.328478965 \ldots\left(\frac{\alpha}{\pi}\right)^{2}+1.181241456 \ldots\left(\frac{\alpha}{\pi}\right)^{3} \\
& -1.5098(384)\left(\frac{\alpha}{\pi}\right)^{4}+4.393(27) \cdot 10^{-12} .
\end{aligned}
$$

The theoretical expressions for the coefficients of this series involve complicated multidimensional integrals the evaluation of which is the main difficulty in the calculation of a series expansion for the muon anomalous magnetic moment. A part of the coefficients in Eq. (II) have been calculated analytically whereas the numbers in the higher order terms have to be evaluated numerically [13, 14].

With the advent of powerful computers a number of problems can now be approached through direct numerical calculations. The trajectories of satellites, for instance, are computed through direct calculations with computers. There are also important applications in Quantum Field Theory (QFT) especially, in the theory of strong interaction formulated on the lattice. However, at present the achieved accuracy is not yet sufficient leaving plenty of room for analytical computations. In addition, calculations in perturbation theory are often formulated in such a way that a numerical approach is not efficient or is unreliable due to the accumulation 
of rounding errors. The situation is quite analogous to the problem of long-time weather forecasts in meteorology where the direct numerical approaches are extremely unstable from the computational point of view. Rounding errors of the numerical evaluation of a large number of Feynman diagrams are such that the final accuracy is low because of huge cancellations between separate contributions of separate diagrams. Although an analytic evaluation is definitely preferable it cannot always be done due to technical difficulties in calculating the relevant Feynman integrals.

In Quantum Field Theory only a very limited number of problems have a full exact solution. Perturbation theory is a general tool for investigating the realistic physical situations. This is especially true in the theory of strong interactions and in the Standard Model in general. The classical examples are the analysis of $e^{+} e^{-}$annihilation into hadrons and the analysis of tau decays where perturbation theory is used in its most advanced form for the calculation up to very high orders of the strong coupling constant [15, 16]. In the latter case such an analysis has lead to the most accurate extraction of strong interaction parameters at low energies [17, 18, 19]. The attempt to extrapolate the calculations to all orders for some limited subsets of diagrams or contributions are also quite useful [20, 21, 22, 23]. The coefficients of the expansions are given by complicated integrals which have a useful graphical representation in terms of Feynman diagrams. Graphical techniques are very efficient and convenient in the bookkeeping of the various loop contributions and are in wide use. In higher orders these graphs have many loops which are associated with the loop integrals. One speaks of multi-loop calculations for the evaluation of the coefficients of the perturbative expansion in QFT. Due to big advances in symbolic computing the most advanced symbolic programs can nowadays generate the terms of the perturbative expansion without actually drawing any graphs. Nevertheless, the terminology has survived. Nowadays the methods of QFT (and its terminology) is starting to penetrate into fields other than high energy physics such as polymer physics, hydro- and aerodynamics and weather forecasts which are much closer to real life. Therefore, the calculation of the complicated integrals associated with Feynman diagrams in a perturbative expansion is important in high energy physics as well as in many other areas of physics.

While the problem of calculating multi-loop integrals will not be solved in a general form in the foreseeable future, there is a continuing interest in the analysis of particular diagrams in the efforts to enlarge the number of known analytical results (as a review, see e.g. 24]). Especially promising in this regard is the analysis of subclasses of diagrams with simpler topological structures but with an arbitrary number of loops. The most promising in this respect is the simple topology of the so-called generalized sunrise diagrams (sometimes also called sunset, 
water melon, banana, or basketball diagrams). They will be referred to as the sunrise-type diagrams in this report. They can be computed for an arbitrary number of loops which makes them an ideal tool to obtain clues to the structure of more complicated multi-loop diagrams [25, 26, 27].

Recently there has been a renewal of interest in the calculation of diagrams of the sunrisetype topology for different numbers of loops involving different loop masses and/or external momenta of the diagram (see e.g. [28, 29, 30, 31]). The classical (or genuine) two-loop sunrise diagram with different values of the internal masses has recently been studied in some detail using the traditional momentum space approach (see e.g. [32, 33, 34, 35, 36] and references therein). In the present report we review the configuration space technique (also called $x$-space technique) for the calculation of sunrise-type diagrams. The configuration space technique is best suited for the topology of the diagram. It considerably reduces the complexity of the calculation and allows for new qualitative and quantitative results for this important particular class of Feynman integrals [25, 37, 38, 39, 40, 41]. Configuration space techniques can be used to verify known results obtained within other techniques both analytically and numerically [42, 43. 44, 45] and to investigate some general features of Feynman diagram calculation [46, 30, 39, 47, 48, 49, 50, 51. We list features of the $x$-space technique in turn with the aim to show how efficient the method is.

The diagrams with sunrise-type topology form a subset of the general set of diagrams with a given number of loops. They appear in various specific physics applications:

- multi-loop calculations in general [52, 53]

- the analysis of static properties of baryons using sum rule techniques in QCD [54, 55, 56, 57, 58, 59, 60, 61, 62, 63.

- the properties of glueballs extracted from the perturbative analysis of two-point correlators 64, 65]

- the multi-particle phase space for multi-body decays of a single-particle phase space 66 . 67. 68

- lattice QCD calculations 69, 70]

- mixing of neutral mesons [1]

- Chiral perturbation theory (ChPT) and effective theories for Goldstone modes in higher orders of the momentum expansion [32, 36, 72] 
- analysis of exotic states in QFT: multi-quark states in QCD or pentaquarks using sum rule techniques [73, 74, 75, 76, 77, 78, 79, 80, 81,

- general questions of QFT: sum rules in two-dimensional QED [82, 83] and properties of baryons in the large $N_{c}$ limit of QCD [84, 85]

- effective potentials for symmetry breaking [86, 87, 88, 89, 90,

- finite temperature calculations [34, 91, 92, 93, 94, 95, 96, 97.

- applications in nuclear physics [98, 99, 100, 101, 102, 103,

- phase space integrals for particles in jets where the momentum along the direction of the jet is fixed [104]

- applications in solid state physics [105]

The principal aim of this report is to assemble all the necessary tools needed for the computation of multi-loop Feynman diagrams with sunrise-type topology. We discuss the whole known spectrum of different methods to analyze these integrals. Amomg these are concise analytical evaluation techniques, expansion in small parameters such as masses and momenta (or inverse masses and momenta), expansions in special kinematical regions as the threshold regime in the Minkowskian domain, integral representations for integrals including their discontinuity across physical cuts in terms of analytic functions of the external momentum, and, finally, efficient, fast, simple and stable numerical procedures. In the Euclidean domain the numerical procedures derived from our representation are efficient and reliable, i.e. stable against error accumulation. In order to acquaint the reader with the tools given given in the report we start with basic notations and relations.

We present a comprehensive report on configuration space techniques including many technical details such that an interested reader can use this report as a practical guide for practical calculations. We have included a great deal of mathematical material such that the report is self-contained to a large degree. In most cases it is not necessary to consult mathematical handbooks in order to understand the calculations. We also give a rather large sample of worked-out examples. The calculational methods used are rather well-suited for further development and can easily be tailored to the further specific needs of the potential user. Using the results of this paper one can create one's own software for an efficient evaluation of the many quantities of interest that can be extracted from the analysis of sunrise-type diagrams. 
The report is organized as follows. In Sec. 2 we briefly summarize some general notions and their application to sunrise-type diagrams. In Sec. 2.1 we introduce the configuration space representation of sunrise-type diagrams and fix our notation. In Sec. 2.2 we specify what is meant by computing a sunrise-type diagram. In Sec. 2.3 we discuss the ultraviolet (UV) divergence structure of sunrise-type diagrams and present recipes to regularize the UV divergences either by subtraction or by dimensional regularization. In Sec. 2.4 we comment on the spectral density of sunrise-type diagrams and its connection to phase space. Sec. 3 is devoted to some explicit configuration space calculations involving both analytical and numerical methods the results of which are compared to previously known results where other calculational techniques have been used. One of the examples is the direct computation of the spectral density of sunrise-type diagrams without taking recourse to Fourier transforms. The results are important for multibody phase space calculations. In Sec. 4 we discuss methods to find asymptotic expansions in different kinematical regimes of mass and/or momentum configurations in the Euclidean domain. Sec. 5 contains a generalization to non-standard propagators and non-scalar cases. In Sec. 6 we generalize the configuration space technique to other topologies. Sec. 7 contains our conclusions. Some of the lengthier formulas are relegated to the appendices, together with useful mathematical material about Bessel functions and Gegenbauer polynomials and a short treatise about cuts and discontinuities as they are occuring in the main text.

\section{Basic notions and relations for sunrise-type diagrams}

Sunrise-type diagrams are graphic representations of the $n$-loop two-point correlation functions in QFT with $(n+1)$ internal propagators connecting the initial and final vertex. The wellstudied genuine sunrise diagram shown in Fig. 1(a) is the leading order perturbative correction to the lowest order propagator in $\phi^{4}$-theory, i.e. it is a two-point two-loop diagram with three internal lines. This diagram emerges as a correction to the Higgs boson propagation in the Standard Model. It also naturally appears in some effective theory approaches to critical phenomena and studies of phase transitions in QFT. The corresponding leading order perturbative correction in $\phi^{3}$-theory is a one-loop diagram which can be considered as a oversimplified case of the prior example.

The two-loop case is a standard starting point for the calculation of radiative corrections in QFT. It emerges in a huge variety of physical applications. Fortunately it can be analytically computed in any desired kinematical regime for any values of the relevant parameters. In this respect it does not present a real challenge as far as multi-loop calculations are concerned. We 


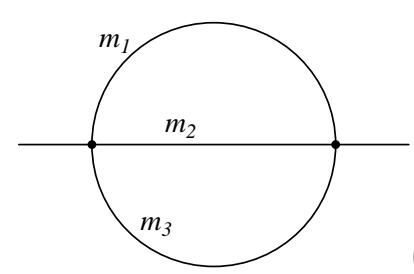

(a)

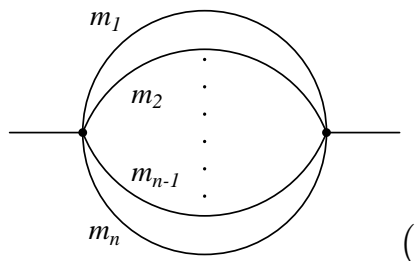

Figure 1: Genuine sunrise (a) and general topology of the class of sunrise-type diagrams (b)

shall, nevertheless, discuss the genuine sunrise diagram in some detail in order to illustrate the efficiency of our configuration space methods. We shall compare our results with known exact analytical results obtained with other techniques. This provides a mutual check on the correctness of the results. A straightforward generalization of this topology to the multi-loop case is a correction to the free propagator in $\phi^{n+2}$-theory that contains $n$ loops and $(n+1)$ internal lines (see Fig. 1(b)).

A subclass of the general sunrise-type diagram shown in Fig. 1(b) is the case when the two external momenta vanish. This subclass is referred to as the subclass of vacuum bubble diagrams with sunrise-type topology which will be referred to as vacuum bubbles. They have a simpler structure than the true sunrise-type diagrams since the number of mass scales is reduced by one for the vacuum bubbles. We shall frequently return to the discussion of vacuum bubbles in the main text.

\subsection{Definitions and notation}

A $n$-loop sunrise-type diagram is a two-point correlation function with $(n+1)$ propagators connecting points $x$ and $y$ and as such is explicitly given by a product of simple propagators built from the basic two-point correlation function $D(x, y, m)$,

$$
\Pi(x, y)=\prod_{i=1}^{n+1} D\left(x, y, m_{i}\right)
$$

(and/or their derivatives if necessary). The quantity $D(x, y, m)$ may be the propagator of a free massive particle with mass $m$ or a more general two-point correlation function. The sunrisetype diagrams are the leading order contribution to a two-point correlation function of two local currents $j_{n+1}(x)$ of the form

$$
j_{n+1}(x)=\mathcal{D}_{\mu_{1}} \phi_{1} \cdots \mathcal{D}_{\mu_{n+1}} \phi_{n+1}
$$

where the fields $\phi_{i}$ have masses $m_{i}$ and where $\mathcal{D}_{\mu}$ is a derivative with multi-index $\mu=\left\{\mu_{1}, \ldots, \mu_{k}\right\}$ standing for $\mathcal{D}_{\mu}=\partial^{k} / \partial x_{\mu_{1}} \ldots \partial x_{\mu_{k}}$. The sunrise-type diagrams are contained in the leading 
order expression for the polarization function

$$
\Pi(x, y)=\left\langle T j_{n+1}(x) j_{n^{\prime}+1}(y)\right\rangle
$$

where the brackets mean quantum mechanical averaging over the ground state which is explicitly given by a product of propagators and/or their derivatives,

$$
\Pi(x, y)=\mathcal{D}_{\mu_{1} \nu_{1}}\left(x, y, m_{1}\right) \cdots \mathcal{D}_{\mu_{n+1} \nu_{n+1}}\left(x, y, m_{n+1}\right)
$$

As the standard case we will consider a translation invariant situation in which the propagator depends on the difference of the arguments only, $D(x, y, m)=D(x-y, m)$. An exception to this important standard case would be the existence of an arbitrary external field. However, in the present report we will not further discuss this possibility. In the standard case one of the vertices can conveniently be placed at the origin, say $y=0$. The propagator is then a function of $x$ only, $D(x, m)$. The basic expression for the sunrise-type diagram in configuration space reads

$$
\Pi(x)=\prod_{i=1}^{n+1} D\left(x, m_{i}\right) .
$$

$D(x, m)$ represents a free propagator of a massive particle with mass $m$ in $D$-dimensional (Euclidean) space-time. It is given by

$$
D(x, m)=\frac{1}{(2 \pi)^{D}} \int \frac{e^{i(p \cdot x)} d^{D} p}{p^{2}+m^{2}}=\frac{(m x)^{\lambda} K_{\lambda}(m x)}{(2 \pi)^{\lambda+1} x^{2 \lambda}}
$$

where we have used $D=2 \lambda+2 . K_{\lambda}(z)$ is the modified Bessel function of the second kind (sometimes also known as McDonald function, see Appendix A defined by Eq. (A8).

The massless propagator can be obtained from Eq. (7) by taking the limit $m \rightarrow 0$ (or more precisely the limit $m x \rightarrow 0$ at fixed $x$ in terms of the dimensionless quantity $m x)$. It reads

$$
D(x, 0)=\frac{1}{(2 \pi)^{D}} \int \frac{e^{i(p \cdot x)} d^{D} p}{p^{2}}=\frac{\Gamma(\lambda)}{4 \pi^{\lambda+1} x^{2 \lambda}} .
$$

and where $\Gamma(\lambda)$ is Euler's Gamma function.

In some physics applications one may have more general basic two-point functions ("modified propagators") for massive particles. An example is the calculation of the three-point function necessary for the determination of a particle form factor at zero momentum transfer when the external momentum of the current vanishes. This momentum configuration reduces the diagram to a sunrise-type diagram with more complicated basic two-point lines (see e.g. [106]). This phenomenon frequently occurs also in calculations of various quantities in Chiral Perturbation Theory (ChPT) [107]. Formally, these two-point functions cannot be considered as propagators 
of a physical particle but emerge as effective basic two-point functions. The simplest modification of the basic propagator which frequently appears in applications is the occurrence of higher powers of the standard propagator. In momentum space it has the form

$$
\tilde{D}^{(\mu)}(p, m)=\frac{1}{\left(p^{2}+m^{2}\right)^{\mu+1}}
$$

For integer $\mu$ these cases appear if one e.g. considers mass derivatives of the propagator as they are needed in certain parameter expansions. For instance, one can consider

$$
\tilde{D}^{\prime}(p, m)=-\frac{\partial}{\partial m^{2}} \frac{1}{\left(p^{2}+m^{2}\right)}
$$

In the same way a derivative of the momentum itself will also increase the power of the propagator. For instance, the second order scalar derivative $\square=\partial_{\mu} \partial^{\mu}$ of the standard propagator will result in

$$
\partial_{\mu} \partial^{\mu} \frac{1}{p^{2}+m^{2}}=\frac{-2}{\left(p^{2}+m^{2}\right)^{2}}+\frac{8 p^{2}}{\left(p^{2}+m^{2}\right)^{3}} .
$$

Higher powers of propagators occur in many applications. Therefore, any calculational technique should be well suited to treat such higher powers. It turns out that the configuration space method very naturally accommodates higher order derivatives.

In configuration space higher powers of the standard propagator with mass $m$ are explicitly given by (cf. Eq. (7D)

$$
\tilde{D}^{(\mu)}(x, m)=\frac{1}{(2 \pi)^{D}} \int \frac{e^{i(p \cdot x)} d^{D} p}{\left(p^{2}+m^{2}\right)^{\mu+1}}=\frac{1}{(2 \pi)^{\lambda+1} 2^{\mu} \Gamma(\mu+1)}\left(\frac{m}{x}\right)^{\lambda-\mu} K_{\lambda-\mu}(m x) .
$$

the additional power of denominator factors (or, equivalently, the number of derivatives in masses) is denoted in the corresponding Feynman diagram by dots placed on the line (though even the power can be non-integer). Note that the modified propagator has the same functional form as the usual propagator. The main difference is the change of the value of the index of the Bessel function $K_{\lambda}(m x) \rightarrow K_{\lambda-\mu}(m x)$. This fact unifies and simplifies the analysis of sunrisetype diagrams that contain more complicated basic propagators (analytical expressions that correspond to one line of the diagrams) as they emerge in some physical applications or are used for the calculation of other diagrams through recurrence relations techniques [108].

\subsection{Momentum versus configuration space representation: Fourier transform in the evaluation of sunrise-type integrals}

The calculation of loop diagrams in perturbative Quantum Field Theory is associated with the evaluation of complicated multi-dimensional integrals, see e.g. [109]. It is obvious that the 
expression for the sunrise-type diagram in configuration space given by Eq. (6) contains no integration at all even if it represents a multi-loop diagram. In this respect it is a kind of tree-level diagram that usually appears in the lowest orders of perturbative QFT. However, "to calculate" a sunrise diagram means in most cases to find certain integrals of Eq. (6) over $x$ with some weight functions depending on the quantity in question. The simplest situation corresponds to a computation of the values of the vacuum bubbles, i.e. expressions for the diagrams without external momenta (or with vanishing external momenta, see e.g. [110]). In case of the sunrise-type topology the value of the vacuum bubble is given by integrals with a simple weight function,

$$
\tilde{\Pi}(0)=\int \Pi(x) d^{D} x=\int D\left(x, m_{1}\right) \cdots D\left(x, m_{n+1}\right) d^{D} x .
$$

This integral provides the simplest example of calculating a sunrise-type diagram. We like to emphasize that this calculation is almost always a must as it allows one to determine the counterterms related to UV divergences of sunrise-type diagrams that always occur in realistic physical models. Due to rotation invariance the integral is one-dimensional as the angular integration can be explicitly done. Thus

$$
\begin{aligned}
\tilde{\Pi}(0) & =\int \Pi(x) d^{D} x=\int D\left(x, m_{1}\right) \cdots D\left(x, m_{n+1}\right) d^{D} x \\
& =\frac{2 \pi^{\lambda+1}}{\Gamma(\lambda+1)} \int D\left(x, m_{1}\right) \cdots D\left(x, m_{n+1}\right) x^{2 \lambda+1} d x
\end{aligned}
$$

The possibility to compute all vacuum bubbles in terms of one-dimensional integrals for any number of loops is a big advantage of the configuration space technique. This guarantees that the complete renormalization program can be explicitly accomplished in terms of onedimensional integrals. It should be clear that this is a huge simplification.

The most familiar example for the evaluation of a sunrise-type diagram is the procedure of finding its value in momentum space, or put differently, the evaluation of its Fourier transform. It corresponds to non-bubble diagrams with two external lines as the integrals then requires the computation of the Fourier transform of $\Pi(x)$,

$$
\tilde{\Pi}(p)=\int \Pi(x) e^{i(p \cdot x)} d^{D} x .
$$

The required integrals are basically scalar integrals. This makes the angular integration in Eq. (13) simple in $D$-dimensional space-time. The needed formula is the integration over the angular variables $d^{D} \hat{x}$ which, in explicit form, reads

$$
\int d^{D} \hat{x} e^{i(p \cdot x)}=2 \pi^{\lambda+1}\left(\frac{p x}{2}\right)^{-\lambda} J_{\lambda}(p x)
$$


where $p=|p|, x=|x|$, and $J_{\lambda}(z)$ is the Bessel function of the first kind. The integration $\int d^{D} \hat{x}$ over the rotationally invariant measure $d^{D} \hat{x}$ on the unit sphere in $D$-dimensional (Eu-

clidean) space-time means integration over angles only. The generalization of Eq. (16) to more complicated integrands with an additional tensor structure $x^{\mu_{1}} \cdots x^{\mu_{k}}$ is straightforward and leads to different orders $J_{\lambda+l}(z)$ of the Bessel function for different irreducible tensors (denoted by the number $l$ ) after angular averaging (integration over angles). The corresponding order of the Bessel function can be inferred from the expansion of the plane wave function $e^{i(p \cdot x)}$ which appears as a weight function upon computing the Fourier transform in a series of Gegenbauer polynomials $C_{l}^{\lambda}(w)$ (see Appendix $(\mathrm{B})$. The Gegenbauer polynomials are orthogonal on a $D$-dimensional unit sphere, and the expansion of the plane wave in terms of Gegenbauer polynomials reads 111

$$
e^{i(p \cdot x)}=\Gamma(\lambda)\left(\frac{p x}{2}\right)^{-\lambda} \sum_{l=0}^{\infty} i^{l}(\lambda+l) J_{\lambda+l}(p x) C_{l}^{\lambda}\left(\frac{(p \cdot x)}{p x}\right) .
$$

Besides the definition of the Gegenbauer polynomials by a characteristic polynomial as given in Eq. (B1), the expansion in Eq. (17) can also serve as a definition of these polynomials.

The representation in Eq. (17) allows one to single out an irreducible tensorial structure from the angular integration given by the Fourier integral in Eq. (13). Integration techniques involving Gegenbauer polynomials for the computation of multi-loop massless diagrams are described in detail in [111] where many useful relations can be found (see also [112] where the calculation in momentum space was considered). One arrives at a representation of the Fourier transform of a sunrise-type diagram that is given in terms of the one-dimensional integral

$$
\begin{aligned}
\tilde{\Pi}(p) & =2 \pi^{\lambda+1} \int_{0}^{\infty}\left(\frac{p x}{2}\right)^{-\lambda} J_{\lambda}(p x) \Pi(x) x^{2 \lambda+1} d x \\
& =2 \pi^{\lambda+1} \int_{0}^{\infty}\left(\frac{p x}{2}\right)^{-\lambda} J_{\lambda}(p x) D\left(x, m_{1}\right) \cdots D\left(x, m_{n+1}\right) x^{2 \lambda+1} d x
\end{aligned}
$$

which is simple to analyze. The representation given by Eq. (18) is quite universal.

\subsection{Regularization and renormalization}

While the configuration space expression for the sunrise-type diagram contains no integration it does not always represent an integrable function of $x$ in configuration space for general values of the space-time dimension $D$. The expression given by Eq. (6) can have non-integrable singularities at small $x$ for a sufficiently large number of propagators if the space-time dimension is $D>2$. 
Upon multiplication of many propagators one may get a function which is singular at small $x$. Each propagator by itself can be considered as a distribution. However, distributions do not form an algebra and their multiplication is not well-defined [113]. In this respect the polarization function $\Pi(x)$ in Eq. (6) is not completely defined as a proper distribution. In attempting to integrate such a function over the whole $x$-space we encounter infinities in the form of ultraviolet (UV) divergences [114. Therefore, the computation of the Fourier transform of $\Pi(x)$ requires regularization (for instance, dimensional regularization) and subtraction. Configuration space provides a nice environment for this.

Note that all these statements depend on the dimension of space-time. The vicinity of $D=2$ is special since the leading singularity of the basic propagator is only logarithmic and the multiplication of propagators does not lead to non-integrable singularities. Therefore, the UV properties of two-dimensional models that are often considered as simplified models in QFT or that emerge in real physical applications as a result of some given approximations [115, 116, 117]. They are much softer than those of realistic four-dimensional models (see e.g. [118]).

In general, however, one has to consider an arbitrary space-time dimension $D$. The modern way of dealing with singularities in QFT in particular in multi-loop calculations is mostly based on dimensional regularization [119, 120, 121] (as a review see [122]). Dimensional regularization is characterized by considering the space-time dimension $D$ to be an arbitrary parameter, for instance $D=D_{0}-2 \varepsilon$ where $D_{0}$ is an integer dimension. ${ }^{1}$ In case of non-integer space-time dimension $D$ the integration is well-defined, while the limit $D \rightarrow D_{0}$ is singular, consisting usually of poles in $D-D_{0}$. These pole parts are extremely simple to handle in configuration space. Indeed, in configuration space the regularization is given by adding the $\delta$-function $\delta(x)$ and its derivatives to $\Pi(x)$. As we will see in the following, this procedure corresponds to adding a polynomial in momentum space (local counterterms according to the Bogoliubov-ParasiukHepp-Zimmermann (BPHZ) theorem [113, 123, 124, 125]). The finite parts can be found numerically for any number of loops by doing a single one-dimensional integration. Therefore, the renormalization in $x$-space becomes

$$
\Pi^{R}(x)=\Pi(x)+C_{0} \delta(x)+C_{1} \square \delta(x)+\ldots+C_{r} \square^{r} \delta(x)
$$

where $\square=\partial_{\mu} \partial^{\mu}$. The coefficients $C_{i}$ are functions of the regularization parameter $\varepsilon$ which are singular in the limit $\varepsilon \rightarrow 0$. The extraction of the coefficients $C_{i}$ is explained in the subsequent

\footnotetext{
${ }^{1}$ The usual choice of the integer space-time dimension $D_{0}$ for real physical applications is $D_{0}=4$, but other space-time dimensions $D_{0}=2, D_{0}=3, D_{0}=5$ or $D_{0}=6$ are being used in other applications. $D_{0}=3$ is natural for near threshold expansions or expansions in the Minkowskian domain in general.
} 
sections for some special cases. The number $r$ of counterterms is determined by dimensional arguments such as power counting and can be easily related to the number of propagators and the space-time dimension. The actual number of poles depends on the mass configuration.

\subsection{The spectrum as discontinuity across the physical cut}

From the physics point of view one important aspect of our analysis of sunrise-type diagrams is the construction of the spectral decomposition of the diagrams. For the two-point correlation function we determine the discontinuity across the physical cut in the complex plane of the squared momentum, $p^{2}=-m^{2} \pm i 0$ which is referred to as the spectral density

$$
\rho(s)=\left.\frac{1}{2 \pi i} \operatorname{Disc} \tilde{\Pi}(p)\right|_{p^{2}=-s}
$$

(cf. Appendix (C). Note that the spectral density of a sunrise-type diagram is finite for any number of loops. It turns out that the configuration space technique allows one to compute the spectral density in a very efficient manner. The analytic structure of the correlator $\Pi(x)$ (or the spectral density of the corresponding polarization operator) can be determined directly in configuration space without having to compute its Fourier transform first. The technique for the direct construction of the spectral density of sunrise-type diagrams introduced in [37] is based on an integral transform in configuration space which in turn is given by the inversion of the relevant dispersion relation.

The dispersion representation (or spectral decomposition) of the polarization function in configuration space has the form

$$
\Pi(x)=\int_{0}^{\infty} \rho(s) D(x, \sqrt{s}) d s=\int_{0}^{\infty} \rho\left(m^{2}\right) D(x, m) d m^{2}
$$

where $\sqrt{s}=m$. This representation was used for sum rule applications in [126, 127] where the spectral density for the two-loop sunrise diagram was given in two-dimensional spacetime [128]. The representation in momentum space is more familiar and is referred to as the Källén-Lehmann representation [129] of the two-point correlation function. In the Euclidean domain it is given by

$$
\tilde{\Pi}(p)=\int_{0}^{\infty} \frac{\rho(s) d s}{s+p^{2}}
$$

This expression can of course be obtained by taking the Fourier transform of both sides of Eq. (201).

With the explicit form of the propagator in configuration space given by Eq. (7), the representation in Eq. (20) turns out to be a particular example of the Hankel transform, namely the 
$K$-transform [130, 131]. Up to inessential factors of $x$ and $m$, Eq. (20) reduces to the generic form of the $K$-transform for a conjugate pair of functions $f$ and $g$,

$$
g(x)=\int_{0}^{\infty} f(y) K_{\nu}(x y) \sqrt{x y} d y
$$

The inverse of this transform is known to be given by

$$
f(y)=\frac{1}{\pi i} \int_{c-i \infty}^{c+i \infty} g(x) I_{\nu}(x y) \sqrt{x y} d x
$$

where $I_{\nu}(x)$ is a modified Bessel function of the first kind and the integration runs along a vertical contour in the complex plane to the right of the right-most singularity of the function $g(x)$ [131]. In order to obtain a representation for the spectral density $\rho\left(m^{2}\right)$ of a sunrise-type diagram in general $D$-dimensional space-time one needs to apply the inverse $K$-transform to the particular case given by Eq. (201). One has

$$
m^{\lambda} \rho\left(m^{2}\right)=\frac{(2 \pi)^{\lambda}}{i} \int_{c-i \infty}^{c+i \infty} \Pi(x) x^{\lambda+1} I_{\lambda}(m x) d x .
$$

From Eq. (23) we obtain an explicit analytical representation for the spectral density $\rho(s)$ as a contour integral of the polarization function which reads

$$
\rho(s)=\frac{(2 \pi)^{\lambda}}{i s^{\lambda / 2}} \int_{c-i \infty}^{c+i \infty} I_{\lambda}(x \sqrt{s}) \Pi(x) x^{\lambda+1} d x
$$

where $I_{\lambda}(z)$ is a modified Bessel function of the first kind and the integration runs along a vertical contour in the complex plane to the right of the right-most singularity of $\Pi(x)$.

The inverse transform given by Eq. (24) completely solves the problem of determining the spectral density $\rho(s)$ of the general class of sunrise-type diagrams by reducing it to the computation of a one-dimensional integral along the contour in a complex plane. This is valid for the general class of sunrise-type diagrams with any number of internal lines and different masses. For $(n+1)$ internal lines with $(n+1)$ equal masses $m$ the spectral density reads

$$
\rho(s)=\frac{m^{\lambda(n+1)}}{i(2 \pi)^{n \lambda+n+1} s^{\lambda / 2}} \int_{c-i \infty}^{c+i \infty} I_{\lambda}(x \sqrt{s})\left(K_{\lambda}(m x)\right)^{n+1} x^{1-n \lambda} d x .
$$

Because the contour can bypass the area of small values of $x$, the integral is finite, as it was stated before. The spectral density in turn can be used to restore the finite part of the correlation function in the momentum space representation. The path traced here is is an alternative to the calculation of the Fourier transform for sunrise-type diagrams.

In the standard, or momentum representation, the polarization function $\tilde{\Pi}(p)$ is calculated from a $n$-loop diagram with $n$-dimensional integrations over the entangled loop momenta. 


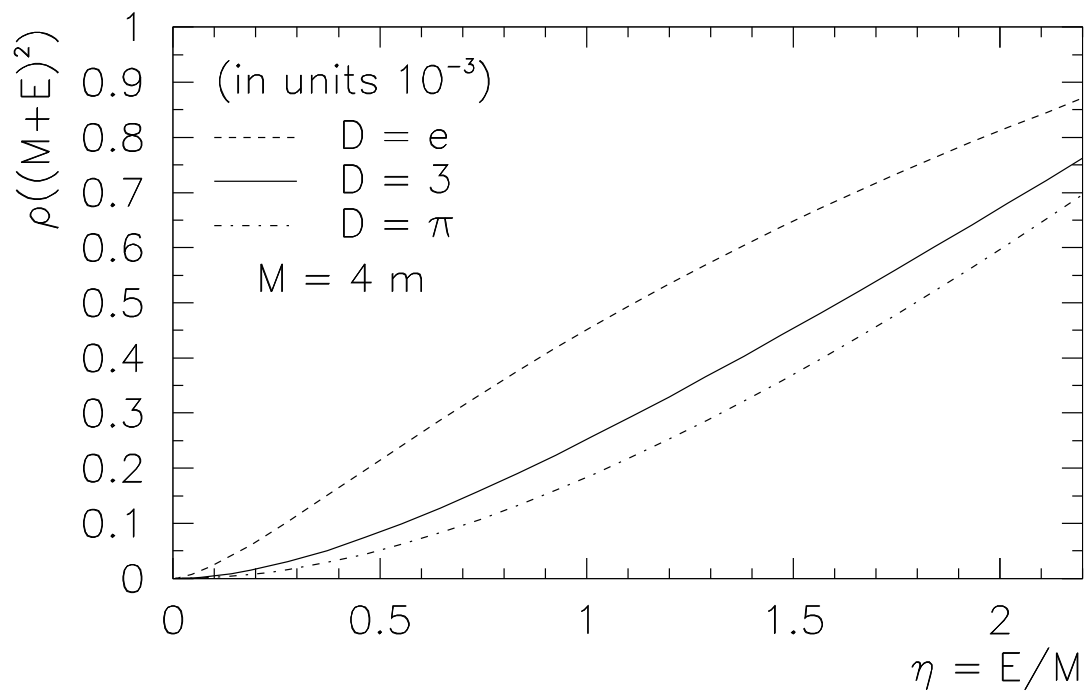

Figure 2: The spectral density for the four-line sunrise-type diagram with equal masses for $D=e=2.718 \ldots, D=3$, and $D=\pi=3.14 \ldots$ space-time dimensions.

It is clear that the momentum space evaluation becomes very difficult when the number of internal lines becomes large.

In order to demonstrate the applicability of our method described in more detail in [37, 38, 39], we show in Fig. 2 the result for the spectral density for the four-line sunrise-type diagram with $D=e=2.718 \ldots, D=3$, and $D=\pi=3.14 \ldots$. We have chosen these exotic values for $D$ to demonstrate the power of configuration space techniques. To the best of our knowledge there is no other method which allows one to compute the spectral density with such completeness and ease, i.e. through a one-dimensional integral. The technique was first suggested in [37] and has been studied in detail in Refs. [66, 41].

Note, finally, that the spectral density of a sunrise-type diagram is a representation of multi-particle phase space with the number of particles equal to the number of lines of the diagram. Our formula for the spectral density is a striking example of how the configurationspace technique can be used to determine the phase space of physical processes. Efficient techniques for the calculation of the multi-particle phase space are important for Monte-Carlo simulations and cross section calculations for multi-particle final states as they will occur at the upcoming Large Hadron Collider (LHC) under construction at CERN. 


\section{3 "Tool box" for a practical analysis}

In this section we give examples and practical prescriptions of how to proceed with the calculation of sunrise-type diagrams containing only a single Bessel function and powers of $x$. The massless case can be solved analytically in closed form for any number $(n+1)$ of internal lines since it contains only a single Bessel function and powers of $x$. The case for one massive line and $n$ massless lines (with two Bessel functions) is completely solvable analytically as well. The first nontrivial situation emerges with two massive lines. In this case a complete analytical solution is unknown in most of the cases, the exception being the case of equal masses that can be integrated analytical through hypergeometric functions. However, when one separates the diagram into a singular or pole part and a finite part, both parts can be calculated either analytically (for the pole part) or numerically (for the finite part) in very general situations. This will be demonstrated in this section.

\subsection{Extraction of poles in dimensional regularization}

Renormalization counterterms can be constructed analytically for general mass configurations and any number of loops. As mentioned earlier, in dimensional regularization the UV divergences become manifest as poles in $\varepsilon \sim D-D_{0}$. In configuration space the UV divergences are related to short distances. In order to analyze the structure of singularities at small $x$, one has to expand the massive propagators at small $x$ (or, effectively, in the masses). In order to obtain the requisite conterterms one then integrates over $x$ which is analytically feasible for $D \neq D_{0}$, i.e. $\varepsilon \neq 0$. Nevertheless, one should ensure convergence also at large $x$ which requires an appropriate IR regularization. Only this guarantees that singularities that emerge after integration are related to small $x$ divergences only (see e.g. 132]). This technical constraint can be met for instance by keeping one (or two) of the massive propagators in the integrand unexpanded. Since the massive propagator falls off exponentially at large $x$, this procedure is sufficient to ensure convergence at large $x$.

The possibility of retaining one propagator unexpanded exists because the integrals of one or a product of two Bessel functions are known analytically [133, 134, 135]. This nice feature provides a tool for obtaining counterterms analytically. The necessary formulae read

$$
\begin{aligned}
\int_{0}^{\infty} x^{\mu-1} K_{\nu}(m x) d x & =2^{\mu-2} m^{-\mu} \Gamma\left(\frac{\mu+\nu}{2}\right) \Gamma\left(\frac{\mu-\nu}{2}\right) \\
\int_{0}^{\infty} x^{2 \alpha-1} K_{\mu}(m x) K_{\mu}(m x) d x & =\frac{2^{2 \alpha-3}}{m^{2 \alpha} \Gamma(2 \alpha)} \Gamma(\alpha+\mu) \Gamma(\alpha) \Gamma(\alpha) \Gamma(\alpha-\mu) .
\end{aligned}
$$


In general, the finite parts of the relevant diagrams resulting from the subtraction of counterterms can be calculated only numerically. Still there are some simple examples where an analytic calculation is feasible. One of these examples is the three-loop sunrise-type diagram with two massive and two massless lines at vanishing external momentum, the three-loop vacuum bubble. There exist an analytical expression for the value of this particular vacuum bubble in configuration space. It is given by

$$
\tilde{\Pi}(0)=\int D(x, m)^{2} D(x, 0)^{2} d^{D} x=\int\left(\frac{(m x)^{\lambda} K_{\lambda}(m x)}{(2 \pi)^{\lambda+1} x^{2 \lambda}}\right)^{2}\left(\frac{\Gamma(\lambda)}{4 \pi^{\lambda+1} x^{2 \lambda}}\right)^{2} d^{D} x
$$

While the angular integration in $D$-dimensional space-time is trivial, the problem of the residual radial integration is solved by using Eq. (27). The result for the integral in Eq. (28) reads

$$
\tilde{\Pi}(0)=\frac{\left(m^{2}\right)^{3 \lambda-1}}{(4 \pi)^{3(\lambda+1)}} \frac{\Gamma(\lambda)^{2} \Gamma(1-\lambda) \Gamma(1-2 \lambda)^{2} \Gamma(1-3 \lambda)}{\Gamma(\lambda+1) \Gamma(2-4 \lambda)} .
$$

This result corresponds to the quantity $M_{1}$ in Ref. [136] where it constitutes the simplest basis element for the computation of massive three-loop diagrams in a general three-loop topology. Again, any number of massless lines can be added.

The result given by Eq. (29) is obtained in a concise form and valid for any dimension $D$. Therefore its pole parts can be explicitly singled out through a direct expansion into a Laurent series near an integer value of the space-time dimension $D$. Taking $D=4-2 \varepsilon$ (i.e. $\lambda=1-\varepsilon$ ) and expanding the result for small $\varepsilon$ one readily finds

$$
\tilde{\Pi}(0)=\frac{\left(m^{2}\right)^{2-3 \varepsilon}}{3(4 \pi)^{3(2-\varepsilon)}}\left(\frac{1}{\varepsilon^{3}}+\frac{7}{2 \varepsilon^{2}}+\frac{25+6 \zeta(2)}{4 \varepsilon}-\frac{5-42 \zeta(2)-56 \zeta(3)}{8}+O(\varepsilon)\right) .
$$

Using this example one can take a closer look at the two-dimensional case. As was stated before, the case $D_{0}=2$ is much softer and the UV divergences are practically absent. Hence one could come to the conclusion that the expression given by Eq. (29) should be perfectly finite at $D=D_{0}=2$. In terms of $D=2 \lambda+2$ the limit corresponds to taking $\lambda \rightarrow 0$. Therefore, one might be surprised to find a singularity $\Gamma(\lambda)^{2}$ in Eq. (29). However, this is not a UV singularity. Because massless propagators are not defined in the two-dimensional world, the limit $\lambda \rightarrow 0$ is ill-defined in this case. Indeed, looking at Eq. (8), the singularity of this sunrise-type diagram is exactly the product of two singular massless propagators. Power counting for the integral expression in Eq. (8) shows that the singularity is caused by the infrared (IR) region. As this is an IR problem, the afore mentioned BPHZ theorem does not apply and the divergence cannot be treated with local counterterms in the usual manner.

Returning to the more general case, a closed-form evaluation (as in Eq. (29)) is not possible and an explicit subtraction is required to separate singular and finite parts. In our case it is 
convenient to use momentum subtraction which in fact is the oldest renormalization method. Momentum subtraction consists of subtracting a polynomial at some fixed momentum point. For massive diagrams the expansion point $p=0$ is safe (i.e. has no infrared (IR) singularity), and the prescription is realized by expanding the function

$$
\left(\frac{p x}{2}\right)^{-\lambda} J_{\lambda}(p x)
$$

(which is the kernel or weight function of the integral transformation in Eq. (18)) in a Taylor series around $p=0$ in terms of a polynomial series in $p^{2}$ (cf. Eq. (A4) in Appendix (A)). The subtraction at order $N$ is achieved by writing

$$
\left[\left(\frac{p x}{2}\right)^{-\lambda} J_{\lambda}(p x)\right]_{N}=\left(\frac{p x}{2}\right)^{-\lambda} J_{\lambda}(p x)-\sum_{k=0}^{N} \frac{(-1)^{k}}{k ! \Gamma(\lambda+k+1)}\left(\frac{p x}{2}\right)^{2 k}
$$

and by keeping $N$ terms in the expansion on the right hand side. After substituting the expansion Eq. (31) into Eq. (18) one obtains the momentum subtracted polarization function

$$
\tilde{\Pi}_{\text {mom }}(p)=\tilde{\Pi}(p)-\left.\sum_{k=0}^{N} \frac{p^{2 k}}{k !}\left(\frac{d}{d p^{2}}\right)^{k} \tilde{\Pi}(p)\right|_{p^{2}=0}
$$

which is finite if the number of subtractions $N$ is sufficiently high. The function $\tilde{\Pi}(p)$ is divergent as well as any of the derivatives on the right hand side of Eq. (32). The divergences require regularization. However, the difference, i.e. the quantity $\tilde{\Pi}_{\mathrm{mom}}(p)$ is finite and independent of any regularization used to give a meaning to each individual term in Eq. (32). Note that the expansion in Eq. (31) is a polynomial in $p^{2}$ in accordance with the general structure of the $R$-operation [114]. The number $N$ of necessary subtractions is determined by the divergence index of the diagram and can be found according to the standard power counting rules. The subtraction at the origin $p=0$ is allowed if there is at least one massive line in the diagram along with an arbitrary number of massless lines. If there are no massive internal lines at all, the corresponding diagram can easily be calculated analytically and the problem of subtraction is trivial. After having performed the requisite subtraction, one can take the limit $D \rightarrow D_{0}$ in Eq. (18) where $D_{0}$ is an integer. The diagram as a whole becomes finite after subtraction which reflects the topology of the sunrise-type diagram: there is no divergent subdiagram in the sense of Bogoliubov.

In selecting the momentum subtraction in order to regularize the singularities we have selected a particular subtraction or renormalization scheme. The coefficients $C_{i}$ in Eq. (19) depend on this choice. While the highest coefficient related to the strongest singularity is unique, the other coefficients are scheme dependent. The standard scheme used in the literature 
is the $\overline{\mathrm{MS}}$ scheme. The transition from the momentum subtraction scheme to the $\overline{\mathrm{MS}}$ scheme is achieved by the familiar redefinition of the measure

$$
d^{D} x \rightarrow\left(4 \pi \mu^{2} e^{-\gamma_{E}}\right)^{\varepsilon} d^{D} x
$$

with $\mu$ being the renormalization scale within dimensional regularization and $\gamma_{E}$ being Euler's constant $\gamma_{E}=0.577 \ldots$

\subsection{Singular and finite parts for particular parameter values of the genuine sunrise: warm-up example demonstrating the technique}

As an example of calculating the counterterms and evaluating the remaining finite part of the diagram we present the special case of the genuine sunrise diagram with three massive lines, i.e. the two-loop sunrise-type diagram. For special values of the external momenta a closedform expression for the genuine sunrise diagram is known within dimensional regularization using momentum space techniques [35]. We shall reproduce this result in the following. The one-dimensional integral to be analyzed is given by

$$
\tilde{\Pi}(p)=2 \pi^{\lambda+1} \int_{0}^{\infty}\left(\frac{p x}{2}\right)^{-\lambda} J_{\lambda}(p x) D\left(x, m_{1}\right) D\left(x, m_{2}\right) D\left(x, m_{3}\right) x^{2 \lambda+1} d x .
$$

To determine the finite part we first use momentum subtraction and split $\tilde{\Pi}(p)$ up into its finite and infinite (but dimensionally regularized) part,

$$
\tilde{\Pi}(p)=\tilde{\Pi}_{\mathrm{mom}}(p)+\tilde{\Pi}_{\text {sing }}(p)
$$

where $\tilde{\Pi}_{\text {mom }}(p)$ is a momentum subtracted polarization function and $\tilde{\Pi}_{\text {sing }}(p)$ is a counterterm in dimensional regularization. Power counting shows that only two subtractions are necessary. The explicit expression for the momentum subtracted polarization function reads

$$
\begin{gathered}
\tilde{\Pi}_{\text {mom }}(p)=2 \pi^{\lambda+1} \int_{0}^{\infty}\left[\left(\frac{p x}{2}\right)^{-\lambda} J_{\lambda}(p x)\right]_{1} D\left(x, m_{1}\right) D\left(x, m_{2}\right) D\left(x, m_{3}\right) x^{2 \lambda+1} d x \\
=2 \pi^{\lambda+1} \int_{0}^{\infty}\left[\left(\frac{p x}{2}\right)^{-\lambda} J_{\lambda}(p x)-\frac{1}{\Gamma(\lambda+1)}+\frac{p^{2} x^{2}}{4} \frac{1}{\Gamma(\lambda+2)}\right] \\
\times D\left(x, m_{1}\right) D\left(x, m_{2}\right) D\left(x, m_{3}\right) x^{2 \lambda+1} d x
\end{gathered}
$$

while the pole part is given by a first order polynomial in $p^{2}$ of the form $A+B p^{2}$. The actual values of the coefficients $A$ and $B$ depend on the regularization scheme that has been used. We 
use dimensional regularization and obtain

$$
\begin{aligned}
\tilde{\Pi}_{\text {sing }}(p)= & A+p^{2} B \\
= & \frac{2 \pi^{\lambda+1}}{\Gamma(\lambda+1)} \int_{0}^{\infty} D\left(x, m_{1}\right) D\left(x, m_{2}\right) D\left(x, m_{3}\right) x^{2 \lambda+1} d x \\
& -p^{2} \frac{2 \pi^{\lambda+1}}{4 \Gamma(\lambda+2)} \int_{0}^{\infty} x^{2} D\left(x, m_{1}\right) D\left(x, m_{2}\right) D\left(x, m_{3}\right) x^{2 \lambda+1} d x
\end{aligned}
$$

With the help of the representation given in Eq. (37) we can apply our strategy in a straightforward manner. The coefficients $A$ and $B$ are simple numbers independent of $p$. They contain divergent parts (regularized within dimensional regularization) and need to be computed only once to recover the function $\tilde{\Pi}(p)$ for any $p$. In the momentum subtracted part one can forego the regularization (it is finite by the $R$-operation) and perform the one-dimensional integration numerically for $D=4$ (i.e. $\lambda=1$ ).

In the particular case of the genuine sunrise diagram the necessary integrals are known analytically in closed form and can be found in integral tables (see e.g. 134]). However, using these tables may not always be convenient and we therefore again present a simplified approach which allows one to deal with even more complicated cases in a simpler manner. Let us specify to the particular case $p=m_{1}+m_{2}-m_{3}$ (which corresponds to a pseudothreshold) where an analytical answer for the integral exists [35. For simplicity we choose $m_{1}=m_{2}=m_{3} / 2=m$. Then $p=0$ and $\tilde{\Pi}_{\text {mom }}(0)=0$ (which is a regular function at this Euclidean point). In this case the counterterm $B p^{2}$ vanishes because the quantity $B$ is finite at finite $\varepsilon$. We thus only have to consider the coefficient $A$. On the other hand our considerations are completely general since for arbitrary $p$ one only requires more terms in the $p^{2}$-expansion. For the special mass configuration considered here the result of 35 reads

$$
\tilde{\Pi}^{\mathrm{ref}}(0)=\pi^{4-2 \varepsilon} \frac{m^{2-4 \varepsilon} \Gamma^{2}(1+\varepsilon)}{(1-\varepsilon)(1-2 \varepsilon)}\left[-\frac{3}{\varepsilon^{2}}+\frac{8 \ln 2}{\varepsilon}-8 \ln ^{2} 2\right]+O(\varepsilon) .
$$

This result can be extracted from the first line of Eq. (37) using the integral tables given in [134]. However, in the general mass case the necessary formulas are rather cumbersome. Even for the special mass configuration considered here they are not so simple. We therefore discuss a short cut which allows one to obtain results immediately without having to resort to integral tables. What we really need is an expansion in $\varepsilon$. Basically we need the integral

$$
\int_{0}^{\infty} D(x, m) D(x, m) D(x, 2 m) x^{2 \lambda+1} d x
$$

which is of the general form

$$
\int_{0}^{\infty} x^{\rho} K_{\mu}(m x) K_{\mu}(m x) K_{\mu}(2 m x) d x
$$


( $\mu=\lambda$ and $\rho=1-\lambda$ in our case). For a product of two Bessel functions in the integrand (without the last one in the above equation) the result of the integration is known analytically and is given by Eq. (27). Let us reduce the problem at hand to Eq. (27) and do our numerical evaluations with functions in four-dimensional space-time where no regularization is necessary. To do so we subtract the leading singularities at small $\xi$ from the last Bessel function in Eq. (40) using the series expansion near the origin given by Eq. (A10). After decomposing the whole answer into finite and singular parts according to $(2 \pi)^{2 D} A=F+S$ (where the total normalization of [35] has been adopted in the definition of $F$ and $S$ ) we find for the singular part

$$
\begin{aligned}
S & =\frac{(2 \pi)^{D} m^{2 \lambda}}{\Gamma(\lambda+1)} \int_{0}^{\infty} x^{2(1-\lambda)-1} K_{\lambda}(m x) K_{\lambda}(m x) \frac{\Gamma(\lambda)}{2}\left[1+\frac{(m x)^{2}}{1-\lambda}-\frac{\Gamma(1-\lambda)}{\Gamma(1+\lambda)}(m x)^{2 \lambda}\right] d x \\
& =\frac{(2 \pi)^{D} m^{2-4 \varepsilon}}{\Gamma(\lambda+1)} \int_{0}^{\infty} \xi^{2 \varepsilon-1} K_{\lambda}(\xi) K_{\lambda}(\xi) \frac{\Gamma(\lambda)}{2}\left[1+\frac{\xi^{2}}{1-\lambda}-\frac{\Gamma(1-\lambda)}{\Gamma(1+\lambda)} \xi^{2 \lambda}\right] d \xi \\
& =\pi^{4-2 \varepsilon} \frac{m^{2-4 \varepsilon} \Gamma^{2}(1+\varepsilon)}{(1-\varepsilon)(1-2 \varepsilon)}\left[-\frac{3}{\varepsilon^{2}}+\frac{8 \ln 2}{\varepsilon}+8\left(2-2 \ln 2-\ln ^{2} 2\right)\right]+O(\varepsilon) .
\end{aligned}
$$

The pole contributions coincide with the result in Eq. (38) while the finite part is different. It is corrected by the finite expression

$$
\begin{aligned}
F= & \frac{(2 \pi)^{D} m^{2 \lambda}}{\Gamma(\lambda+1)} \int_{0}^{\infty} x^{2(1-\lambda)-1} K_{\lambda}(m x) K_{\lambda}(m x) \\
& \times\left\{(m x)^{\lambda} K_{\lambda}(2 m x)-\frac{\Gamma(\lambda)}{2}\left[1+\frac{(m x)^{2}}{1-\lambda}-\frac{\Gamma(1-\lambda)}{\Gamma(1+\lambda)}(m x)^{2 \lambda}\right]\right\} d x .
\end{aligned}
$$

Because $F$ is finite (no strong singularity at small $x$ ) one can put $\lambda=1$ to obtain

$$
F=16 \pi^{4} m^{2} \int_{0}^{\infty} \frac{d x}{x} K_{1}(x) K_{1}(x)\left\{x K_{1}(2 x)-\frac{1}{2}\left[1+x^{2}\left(-1+2 \gamma_{E}+2 \ln x\right)\right]\right\} .
$$

Doing the numerical integration in Eq. (43) results in the expression

$$
F=16 \pi^{4} m^{2}[-0.306853 \ldots]
$$

One can restore all $\varepsilon$-dependence in the normalization factors as in Eqs. (38) and (41) because $F$ is not singular in $\varepsilon$, so that this change of normalization is absorbed by the $O(\varepsilon)$ symbol. One obtains

$$
F=\pi^{4-2 \varepsilon} \frac{m^{2-4 \varepsilon} \Gamma^{2}(1+\varepsilon)}{(1-\varepsilon)(1-2 \varepsilon)}[-0.306853 \ldots]+O(\varepsilon) .
$$

Adding $F$ from Eq. (45) to $S$ from Eq. (41) one obtains a numerical result for the finite part of Eq. (38). In order to establish the full coincidence with the result of Eq. (38), note that

$$
-0.306853 \ldots=-(1-\ln 2) \times 1.0000 \ldots
$$


Of course we have used the analytical expression $(1-\ln 2)$ for the numerical quantity $0.306853 \ldots$ for illustrative reasons because we knew the final answer.

We emphasize that there is nothing new in computing the polarization function related to this diagram at any $p$. Some finite part appears from the momentum subtracted polarization function. One then needs another counterterm for non-zero $p^{2}$. The computation of these additional terms proceeds in analogy to what has been done before. It is even simpler because the singularity at small $x$ is weaker and only one subtraction of the Bessel function is necessary. This computational technique works for any complex value $p^{2}$.

\subsection{Three-loop sunrise-type diagram for special momenta}

In a similar manner one can treat the three-loop sunrise-type diagram with two equal masses $M$, one mass $m$ with values between zero and $M$ and a massless line for outer momentum $p^{2}=-m^{2}$. This particular sunrise-type diagram was analyzed by Mastrolia [29] whose results we confirm. The starting integral expression reads

$$
\begin{aligned}
\tilde{\Pi}(p) & =2 \pi^{\lambda+1} \int_{0}^{\infty}\left(\frac{p x}{2}\right)^{-\lambda} J_{\lambda}(p x) D(x, M) D(x, M) D(x, m) D(x, 0) x^{2 \lambda+1} d x \\
& =\frac{M^{2 \lambda} m^{\lambda} \Gamma(\lambda)}{2(2 \pi)^{3(\lambda+1)}} \int_{0}^{\infty}\left(\frac{p x}{2}\right)^{-\lambda} J_{\lambda}(p x) K_{\lambda}(M x) K_{\lambda}(M x) K_{\lambda}(m x) x^{1-3 \lambda} d x
\end{aligned}
$$

The integral has the same form as in the previous example. It is obvious that we can proceed in the same way as before: first expand the integrand in $p$ and $m$ in order to find the counterterms, and second determine the finite contribution for $p^{2}=-m^{2}$ numerically. However, in this case we have to expand the Bessel function $J_{\lambda}(p x)$ up to the second order in $p^{2}$. For the singular part we obtain

$$
\begin{aligned}
\tilde{\Pi}_{\text {sing }}(p)= & A+p^{2} B+\frac{1}{2} p^{4} C \\
= & \frac{2 \pi^{\lambda+1}}{\Gamma(\lambda+1)} \int_{0}^{\infty} D\left(x, m_{1}\right) D\left(x, m_{2}\right) D\left(x, m_{3}\right) x^{2 \lambda+1} d x \\
& -p^{2} \frac{2 \pi^{\lambda+1}}{4 \Gamma(\lambda+2)} \int_{0}^{\infty} x^{2} D\left(x, m_{1}\right) D\left(x, m_{2}\right) D\left(x, m_{3}\right) x^{2 \lambda+1} d x \\
& +p^{4} \frac{2 \pi^{\lambda+1}}{32 \Gamma(\lambda+3)} \int_{0}^{\infty} x^{4} D\left(x, m_{1}\right) D\left(x, m_{2}\right) D\left(x, m_{3}\right) x^{2 \lambda+1} d x
\end{aligned}
$$

where

$$
A=\frac{2 M^{4-6 \varepsilon}}{(4 \pi)^{3(2-\varepsilon)} \Gamma(2-2 \varepsilon) \Gamma(2-\varepsilon)}\left(\frac{1+2 y^{2}}{6 \varepsilon^{3}}+\frac{2+8 y^{2}-y^{4}-24 y^{2} \ln y}{24 \varepsilon^{2}}\right.
$$




$$
\begin{aligned}
& \left.+\frac{1}{\varepsilon}\left(-\frac{3}{16}\left(2+y^{4}\right)+\frac{2}{3}\left(1+2 y^{2}\right) \zeta(2)-y^{2} \ln y+\frac{1}{4} y^{4} \ln y+y^{2} \ln ^{2} y\right)\right), \\
B= & \frac{2 M^{2-6 \varepsilon}}{(4 \pi)^{3(2-\varepsilon)} \Gamma(2-2 \varepsilon) \Gamma(3-\varepsilon)}\left(\frac{2+y^{2}}{12 \varepsilon^{2}}+\frac{2+y^{2}-6 y^{2} \ln y}{12 \varepsilon}\right), \\
C= & \frac{2 M^{-6 \varepsilon}}{(4 \pi)^{3(2-\varepsilon)} \Gamma(2-2 \varepsilon) \Gamma(4-\varepsilon)}\left(\frac{1}{6 \varepsilon}\right)
\end{aligned}
$$

and $y=m / M$.

\subsection{Infinite parts of sunrise-type diagrams with different masses at any number of loops: general techniques}

If one deals with sunrise-type diagrams with different masses one has to be careful to use a symmetric subtraction procedure. Otherwise one looses the symmetry of the original diagram. Such an asymmetry would arise if one would expand one of the Bessel functions in the integrand of the sunrise-type diagram as was done in the previous example for the massive line with mass $m$. If one wants to obtain a result which is symmetric under the exchange of the masses $m_{i}$ as expected from the topology of the diagram and from the initial form of the integral we have to subtract a counterterm which is symmetric in these masses. In calculating the genuine sunrise diagram one can follow this procedure by using a damping function such as $e^{-\mu^{2} x^{2}}$ to suppress contributions for large values of $x$, corresponding to IR singularities. This is done by introducing the factor $1=e^{\mu^{2} x^{2}} e^{-\mu^{2} x^{2}}$ into the integrand. In this case all massive propagators along with the factor $e^{\mu^{2} x^{2}}$ can be expanded in terms of small $x$ values. The expansion of the propagators is straightforward and can be obtained from Eqs. (A4) and (A10) in Appendix $\mathbb{A}$ The final integration can then be done formally by using the identity

$$
\int_{0}^{\infty} x^{p-1} e^{-\mu^{2} x^{2}} d x=\frac{1}{2} \mu^{-p} \Gamma(p / 2)
$$

which is nothing but the definition of Euler's Gamma function.

As an illustration we compute the four-loop bubble diagram which was also calculated by Laporta [137] whose results we confirm. In the sample results below we extract the normalization factor

$$
\mathcal{N}_{n}=\left(\frac{(4 \pi)^{2-\varepsilon}}{\Gamma(1+\varepsilon)}\right)^{n}
$$

for the $n$-loop (or $(n+1)$-line) sunrise-type diagram. Extraction of this factor renders all pole parts to be rational numbers. The results of the calculation are

$$
\mathcal{N}_{1} \tilde{\Pi}_{1}\left(p^{2}\right)=\frac{1}{\varepsilon}+O\left(\varepsilon^{0}\right),
$$




$$
\begin{aligned}
& \mathcal{N}_{2} \tilde{\Pi}_{2}\left(p^{2}\right)=m^{2}\left\{-\frac{3}{2 \varepsilon^{2}}-\frac{9}{2 \varepsilon}\right\}-\frac{p^{2}}{4 \varepsilon}+O\left(\varepsilon^{0}\right), \\
& \mathcal{N}_{3} \tilde{\Pi}_{3}\left(p^{2}=0\right)=m^{4}\left\{\frac{2}{\varepsilon^{3}}+\frac{23}{3 \varepsilon^{2}}+\frac{35}{2 \varepsilon}\right\}+O\left(\varepsilon^{0}\right), \\
& \mathcal{N}_{3} \tilde{\Pi}_{3}\left(p^{2}=-m^{2}\right)=m^{4}\left\{\frac{2}{\varepsilon^{3}}+\frac{22}{3 \varepsilon^{2}}+\frac{577}{36 \varepsilon}\right\}+O\left(\varepsilon^{0}\right), \\
& \mathcal{N}_{4} \tilde{\Pi}_{4}\left(p^{2}=0\right)=m^{6}\left\{-\frac{5}{2 \varepsilon^{4}}-\frac{35}{3 \varepsilon^{3}}-\frac{4565}{144 \varepsilon^{2}}-\frac{58345}{864 \varepsilon}\right\}+O\left(\varepsilon^{0}\right), \\
& \mathcal{N}_{4} \tilde{\Pi}_{4}\left(p^{2}=-m^{2}\right)=m^{6}\left\{-\frac{5}{2 \varepsilon^{4}}-\frac{45}{4 \varepsilon^{3}}-\frac{4255}{144 \varepsilon^{2}}-\frac{106147}{1728 \varepsilon}\right\}+O\left(\varepsilon^{0}\right), \\
& \mathcal{N}_{5} \tilde{\Pi}_{5}\left(p^{2}=0\right)=m^{8}\left\{\frac{3}{\varepsilon^{5}}+\frac{33}{2 \varepsilon^{4}}+\frac{1247}{24 \varepsilon^{3}}+\frac{180967}{1440 \varepsilon^{2}}+\frac{898517}{3456 \varepsilon}\right\}+O\left(\varepsilon^{0}\right), \\
& \mathcal{N}_{5} \tilde{\Pi}_{5}\left(p^{2}=-m^{2}\right)=m^{8}\left\{\frac{3}{\varepsilon^{5}}+\frac{16}{\varepsilon^{4}}+\frac{49}{\varepsilon^{3}}+\frac{6967}{60 \varepsilon^{2}}+\frac{1706063}{7200 \varepsilon}\right\}+O\left(\varepsilon^{0}\right), \\
& \mathcal{N}_{6} \tilde{\Pi}_{6}\left(p^{2}=0\right)=m^{10}\left\{-\frac{7}{2 \varepsilon^{6}}-\frac{133}{6 \varepsilon^{5}}-\frac{238}{3 \varepsilon^{4}}-\frac{77329}{360 \varepsilon^{3}}\right. \\
&\left.-\frac{21221921}{43200 \varepsilon^{2}}-\frac{2596372387}{2592000 \varepsilon}\right\}+O\left(\varepsilon^{0}\right) .
\end{aligned}
$$

The coefficient of the leading singularity in $\varepsilon$ is independent of $p^{2}$. In Appendix D we list results for unequal mass configurations up to four-loop order. When setting all masses equal the results of the general mass case can be seen to agree with the above equal mass results.

\subsection{Finite part for the genuine sunrise with three different masses}

In configuration space the genuine sunrise diagram with three different masses $m_{1}, m_{2}$, and $m_{3}$ is given by

$$
\Pi(x)=D\left(x, m_{1}\right) D\left(x, m_{2}\right) D\left(x, m_{3}\right) .
$$

The Fourier transform of this polarization function in the Euclidean domain reads

$$
\tilde{\Pi}(p)=2 \pi^{\lambda+1} \int_{0}^{\infty}\left(\frac{p x}{2}\right)^{-\lambda} J_{\lambda}(p x) D\left(x, m_{1}\right) D\left(x, m_{2}\right) D\left(x, m_{3}\right) x^{2 \lambda+1} d x .
$$

In dimensional regularization the singular parts $(D=4-2 \varepsilon$, i.e. $\lambda=1-\varepsilon)$ are given by

$$
\tilde{\Pi}_{\text {sing }}(p)=\frac{\mu^{-4 \varepsilon}}{\pi^{4-2 \varepsilon}}\left\{-\frac{m_{1}^{2}+m_{2}^{2}+m_{3}^{2}}{512 \varepsilon^{2}}+\frac{1}{\varepsilon}\left(\sum_{i=1}^{3} \frac{m_{i}^{2} \ln \left(m_{i} e^{\gamma \prime} / 2 \mu\right)}{128}-\frac{p^{2}}{1024}\right)\right\}
$$

where $\gamma^{\prime}=\gamma_{E} / 2-3 / 4$. The counterterms for the Fourier transform are obtained as explained before by introducing $1=e^{\mu^{2} x^{2}} e^{-\mu^{2} x^{2}}$ and expanding the relevant Bessel functions together with $e^{\mu^{2} x^{2}}$. This is the reason for the explicit dependence on the arbitrary parameter $\mu$. In this way one readily finds the pole parts for any diagram with any mass configuration, i.e. one finds the coefficients $C_{i}$ of the counterterms in Eq. (19). Even if an external momentum goes through 
the diagram, the pole parts can be still calculated easily since they are given by integrals over the expanded integrand. The momentum subtracted finite part is given by

$$
\tilde{\Pi}_{\text {fin }}(p)=2 \pi^{\lambda+1} \int_{0}^{\infty}\left[\left(\frac{p x}{2}\right)^{-\lambda} J_{\lambda}(p x)\right]_{N} D\left(x, m_{1}\right) D\left(x, m_{2}\right) D\left(x, m_{3}\right) x^{2 \lambda+1} e^{\mu^{2} x^{2}} e^{-\mu^{2} x^{2}} d x
$$

where $N$ is the order of the expansion needed to ensure integrability. Naturally $N$ has the same order as the one used earlier in order to extract the counterterms. It is obvious, though, that the expansion can terminate at positive powers of $x$. Finally, one can set $\varepsilon=0$ to calculate the finite part numerically. As a technical remark note that most numerical integration routines will run into problems if the upper limit extends to infinity. However, already for values of the order $x \sim 1$ the integrand is negligibly small, so that the integration can be cut off at this point. Also the region around the origin might cause trouble for the convergence of the numerical integration. In this case the integration interval can be subdivided with an interval close to the origin, and the whole integrand in this subinterval can be expanded in $x$ and integrated analytically. For specific values of $p^{2}, m_{1}, m_{2}$, and $m_{3}$ we were able to reproduce results given in the literature (see e.g. results given in Refs. [43, 138]).

\subsection{Calculation of the finite part: further examples}

The examples presented so far are well-known and have been obtained before by using techniques different from the ones presented here. While we can numerically compute any sunrisetype diagram with any arbitrary number of internal massive lines it is not always possible to find corresponding analytical expressions to compare with. Beyond two loops only a few exam-

ples can be found in the literature. For instance, we have calculated the result for the vacuum bubble diagram shown in Fig. 3 (see also Sec. 5.4). For $p \rightarrow 0$ we obtain (cf. Eq. (A4))

$$
\left(\frac{p x}{2}\right)^{-\lambda} J_{\lambda}(p x) \rightarrow \frac{1}{\Gamma(\lambda+1)}
$$

One therefore has

$$
\tilde{\Pi}(0)=\frac{2 \pi^{\lambda+1}}{\Gamma(\lambda+1)} \int_{0}^{\infty}\left(D^{(1)}(x, m)\right)^{3} D(x, m) x^{2 \lambda+1} d x
$$

where $D^{(1)}(x, m)$ is defined in Eq. (12). In order to compare with the literature [136], we multiply with a relative factor $\mathcal{N}_{3} m^{2+6 \varepsilon}$ (cf. Eq. (50) ) to obtain

$$
B_{\varepsilon}=\mathcal{N}_{3} m^{2+6 \varepsilon} \tilde{\Pi}(0)=\frac{2^{2-2 \varepsilon}}{(1-\varepsilon) \Gamma(1+\varepsilon)^{3} \Gamma(1-\varepsilon)} \int_{0}^{\infty} K_{-\varepsilon}^{3}(x) K_{1-\varepsilon}(x) x^{2+2 \varepsilon} d x .
$$




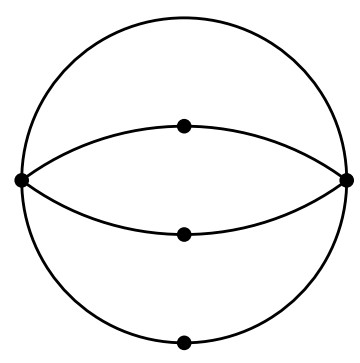

Figure 3: Three-loop massive sunrise-type bubble. A dot on a line corresponds to one differentiation with respect to the propagator mass squared

The integral remains finite even for $\varepsilon \rightarrow 0$ and, after finite integration, we obtain the numerical result

$$
B_{0}=4 \int_{0}^{\infty} K_{0}^{3}(x) K_{1}(x) x^{2} d x=2.1035995805 \ldots
$$

A comparison with the results presented in Ref. [136] shows full numerical agreement. Since we know that the quantity $B_{0}$ contains only a single transcendental number, namely $\zeta(3)$, where $\zeta(z)$ is Riemann's $\zeta$-function [136, 139], we can take its numerical value $\zeta(3)=1.202056903 \ldots$ to obtain the result

$$
B_{0}=\zeta(3) \cdot 1.7500000 \ldots=\frac{7}{4} \zeta(3)
$$

which is nothing but the analytical result given in Ref. [136].

We conclude that the configuration space method is simple and allows one to numerically compute diagrams with sunrise-type topology in a very efficient manner. When the structure of the transcendentality of the result is known for a particular diagram (as in the latest example where only $\zeta(3)$ is present) or when it can be obtained from an educated guess looking at the topology of the diagram [140] our numerical technique can be used to restore the rational coefficients of these transcendentalities which can be thought of as elements of the basis for a class of diagrams.

\subsection{Higher order expansion in $\varepsilon$ : general techniques and the four-loop vacuum bubble}

After having determined the singular parts of the Laurent series expansion using the damping factor method what remains to be done is to calculate the coefficients of the positive powers of $\varepsilon$ in the $\varepsilon$-expansion including the finite $\varepsilon^{0}$ term. Higher order terms in the $\varepsilon$-expansion are needed if the sunrise-type diagram is inserted into a divergent diagram or when one is using 
the integration-by-parts recurrence relation which can generate inverse powers of $\varepsilon$. In order to determine these higher order terms one needs to resort to the Bessel function method. What is technically needed is to develop a procedure for the $\varepsilon$-expansion of Bessel functions.

The $O(\varepsilon)$ term can be obtained analytically. Indeed, within dimensional regularization the propagator in configuration space contains Bessel functions with non-integer index depending on the regularization parameter $\varepsilon$, as they occurred for instance in Sec. 3.6 at an intermediate step. In order to expand the Bessel function in the parameter $\varepsilon$ entering its index, we use Eq. (A13) for the derivative of the modified Bessel function of the second kind with respect to its index near integer values of this index. For instance, we obtain

$$
\begin{aligned}
K_{-\varepsilon}(x) & =K_{0}(x)+O\left(\varepsilon^{2}\right) \\
K_{1-\varepsilon}(x) & =K_{1}(x)-\frac{\varepsilon}{x} K_{0}(x)+O\left(\varepsilon^{2}\right) \\
K_{2-\varepsilon}(x) & =K_{2}(x)-\frac{2 \varepsilon}{x} K_{1}(x)-\frac{2 \varepsilon}{x^{2}} K_{0}(x)+O\left(\varepsilon^{2}\right) \quad \ldots
\end{aligned}
$$

For the more general cases one can use the integral representation

$$
K_{\nu}(z)=\int_{0}^{\infty} \cosh (\nu t) e^{-z \cosh t} d t
$$

for the index expansion. However, one can only obtain numerical results in this case. In expanding the integrand, we obtain

$$
\begin{aligned}
K_{-\varepsilon}(z) & =\sum_{n=0}^{\infty} \frac{\varepsilon^{2 n}}{(2 n) !} f_{2 n}(z) \\
K_{1-\varepsilon}(z) & =\sum_{n=0}^{\infty} \frac{\varepsilon^{2 n}}{(2 n) !} a_{2 n}(z)-\sum_{n=0}^{\infty} \frac{\varepsilon^{2 n+1}}{(2 n+1) !} b_{2 n+1}(z)
\end{aligned}
$$

where

$$
\begin{aligned}
& f_{k}(z)=\int_{0}^{\infty} t^{k} e^{-z \cosh t} d t \\
& a_{k}(z)=\int_{0}^{\infty} t^{k} \cosh t e^{-z \cosh t} d t \\
& b_{k}(z)=\int_{0}^{\infty} t^{k} \sinh t e^{-z \cosh t} d t
\end{aligned}
$$

Due to the recurrence relations in the index $\nu$ for the family $K_{\nu}(z)$ (cf. Sec. 5.6) the two formulas in Eq. (62) suffice to calculate the Bessel function $K_{\nu}(z)$ for any $\nu$. The two functions $a_{k}(z)$ and $b_{k}(z)$ in Eq. (62) are in turn related to the functions $f_{k}(z)$,

$$
a_{k}(z)=-\frac{d}{d z} f_{k}(z), \quad b_{k}(z)=\frac{k}{z} f_{k-1}(z) .
$$




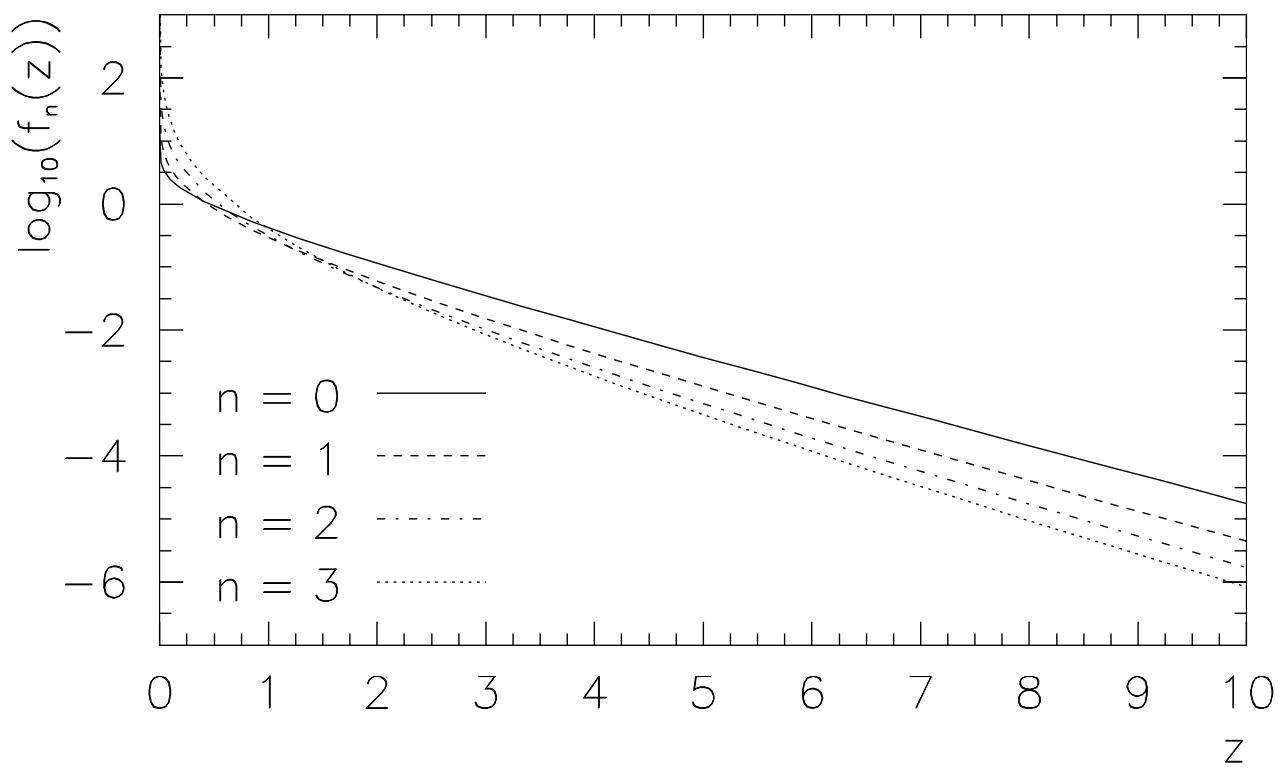

Figure 4: Comparison of functions $f_{n}(z)$ for different values of $n$, plotted on a logarithmic scale

Using these relations, we obtain

$$
K_{1-\varepsilon}(z)=-\sum_{n=0}^{\infty} \frac{\varepsilon^{2 n}}{(2 n) !}\left(\frac{d}{d z}+\frac{\varepsilon}{z}\right) f_{2 n}(z) .
$$

The family of functions $f_{k}(z)$ is rather close to the original set of Bessel functions $K_{\nu}(z)$ (cf. Fig. (4) and can easily be studied both analytically and numerically. The limits at $z \rightarrow 0$ and $z \rightarrow \infty$ are known analytically and are simple. They allow for an efficient interpolation for intermediate values of the argument. The functions $f_{k}(z)$ satisfy the differential equation

$$
\left(\frac{d^{2}}{d z^{2}}+\frac{1}{z} \frac{d}{d z}-1\right) f_{k}(z)=\frac{k(k-1)}{z^{2}} f_{k-2}(z)
$$

related to the Bessel differential equation. The small $z$ behaviour

$$
f_{k}(z)=\frac{1}{k+1} \ln ^{k+1}\left(\frac{1}{z}\right)\left(1+O\left(\frac{1}{\ln (z)}\right)\right)
$$

can be found by using yet another representation for the function $f_{k}(z)$,

$$
f_{k}(z)=\int_{1}^{\infty} \frac{e^{-z u}}{\sqrt{u^{2}-1}} \ln ^{k}\left(u+\sqrt{u^{2}-1}\right) d u
$$

or directly from the behaviour of the function $K_{\nu}(x)$ at small $x$,

$$
K_{-\varepsilon}(x)=\frac{1}{\varepsilon} \sinh (\varepsilon \ln (1 / x)) .
$$


While in four-dimensional space-time the massive propagator contains the Bessel function $K_{1-\varepsilon}(z)$, the basic function in $D=2$ dimensional space-time is the Bessel function $K_{-\varepsilon}(z)$ since the propagator reads

$$
\left.D(x, m)\right|_{D=2}=\frac{1}{2 \pi} K_{0}(m x) .
$$

As an example for the numerical evaluation of the $\varepsilon$-expansion using Bessel functions we consider a toy model integral related to the one-loop case in two dimensions. We select this example because the integral is finite and analytically known, so that we can compare our numerical calculation with the exact answer. Using Eq. (27), we obtain

$$
2 \int K_{-\varepsilon}(x) K_{-\varepsilon}(x) x d x=\Gamma(1+\varepsilon) \Gamma(1-\varepsilon) .
$$

The expansion in $\varepsilon$ is given by

$$
\Gamma(1-\varepsilon) \Gamma(1+\varepsilon)=\frac{\pi \varepsilon}{\sin (\pi \varepsilon)}=1+\frac{\pi^{2} \varepsilon^{2}}{6}+\frac{7 \pi^{4} \varepsilon^{4}}{360}+O\left(\varepsilon^{6}\right)
$$

On the other hand, we can use the expansion

$$
K_{-\varepsilon}(x)=f_{0}(x)+\frac{\varepsilon^{2}}{2} f_{2}(x)+\frac{\varepsilon^{4}}{24} f_{4}(x)+O\left(\varepsilon^{6}\right)
$$

to rewrite the integral in the form

$$
\begin{aligned}
2 \int_{0}^{\infty} K_{-\varepsilon}(x) K_{-\varepsilon}(x) x d x= & 2 \int_{0}^{\infty} f_{0}(x)^{2} x d x+2 \varepsilon^{2} \int_{0}^{\infty} f_{0}(x) f_{2}(x) x d x \\
& +\frac{\varepsilon^{4}}{6} \int_{0}^{\infty} f_{0}(x) f_{4}(x) x d x+\frac{\varepsilon^{4}}{2} \int_{0}^{\infty} f_{2}(x)^{2} x d x+O\left(\varepsilon^{6}\right) .
\end{aligned}
$$

Using the explicit expressions for the functions $f_{k}$ we have checked by numerical integration that the identities

$$
\begin{aligned}
2 \int_{0}^{\infty} f_{0}(x)^{2} x d x & =1, \\
2 \int_{0}^{\infty} f_{0}(x) f_{2}(x) x d x & =\frac{\pi^{2}}{6}, \\
\frac{1}{6} \int_{0}^{\infty}\left(f_{0}(x) f_{4}(x)+3 f_{2}(x)^{2}\right) x d x & =\frac{7 \pi^{4}}{360}
\end{aligned}
$$

are valid numerically with very high degree of accuracy. We have implemented our algorithm for the $\varepsilon$-expansion of sunrise-type diagrams as a simple code in Wolfram's Mathematica system for symbolic manipulations and checked its work-ability and efficiency.

When analyzing the $\varepsilon$-expansion one realizes that integrals over products of modified Bessel functions of the second kind result in integrals over products of functions $f_{k}(z)$. But because 
the analytical behaviour of the functions $f_{k}(z)$ is quite similar to the original Bessel functions, the numerical integration is again easy to perform. For example, in the case of the four-loop bubble diagram, the integral

$$
\tilde{\Pi}_{4}\left(p^{2}=0\right)=\int D(x, m)^{5} d^{D} x
$$

can be calculated by subtracting the expansion (cf. Appendix E)

$$
\begin{aligned}
\Delta(x)=\frac{1}{4 \pi^{\lambda+1} x^{2 \lambda}}\{\Gamma(\lambda) & +\left(\left(\frac{x}{2}\right)^{2} \frac{\Gamma(\lambda)}{1-\lambda}-\left(\frac{x}{2}\right)^{2 \lambda} \frac{\Gamma(1-\lambda)}{\lambda}\right) \\
+ & \left.\left(\frac{x}{2}\right)^{2}\left(\left(\frac{x}{2}\right)^{2} \frac{\Gamma(\lambda)}{2(1 \lambda)(2-\lambda)}-\frac{x^{2 \lambda}}{2} \frac{\Gamma(1-\lambda)}{\lambda(\lambda+1)}\right)\right\}
\end{aligned}
$$

from each of the propagators except for one which we keep as IR regulator. We obtain

$$
\begin{aligned}
\tilde{\Pi}_{4}(0)= & \int D(x, m)(D(x, m)-\Delta(x)+\Delta(x))^{4} d^{D} x \\
= & \int D(x, m)\left((D(x, m)-\Delta(x))^{4}+4(D(x, m)-\Delta(x))^{3} \Delta(x)\right. \\
& \left.+6(\Delta(x, m)-\Delta(x))^{2} \Delta(x)^{2}+4(D(x, m)-\Delta(x)) \Delta(x)^{3}+\Delta(x)^{4}\right) d^{D} x .
\end{aligned}
$$

The last two terms in Eq. (178) can be integrated analytically and can be expanded to an arbitrary order in $\varepsilon$. They contain the singular contributions, i.e. all poles in $\varepsilon$ and are expressible through Euler's $\Gamma$-functions. As expected, the pole part coincides with the expression in Eq. (51). Because the analytical expression (as given up to order $\varepsilon^{3}$ in Appendix $\mathrm{F}$ ) is rather lengthy, we present only its numerical evaluation,

$$
\begin{gathered}
\mathcal{N}_{4} \tilde{\Pi}_{4}^{\text {ana }}(0)=m^{6}\left(-2.5 \varepsilon^{-4}-11.6666667 \varepsilon^{-3}-31.701389 \varepsilon^{-2}-67.528935 \varepsilon^{-1}\right. \\
-15871.965743-142923.10240 \varepsilon-701868.64762 \varepsilon^{2} \\
\left.-2486982.5547 \varepsilon^{3}+O\left(\varepsilon^{4}\right)\right) .
\end{gathered}
$$

The remaining first three terms in Eq. (78) can be integrated numerically for $D=4$ using the expansion of the Bessel functions in terms of the functions $a_{k}(z)$ and $b_{k}(z)$ [141]. No regularization is necessary since these contributions are regular at small $x$. The analytical expression for the functions to be integrated is again rather long. The zeroth order $\varepsilon$-coefficient is found in Appendix $G$ (see also the discussion of the integration procedure given in Appendix G). However, as shown in Fig. 5. the integrands themselves show a very smooth behaviour which renders the numerical integration rather simple. We obtain

$\mathcal{N}_{4} \tilde{\Pi}_{4}^{\text {num }}(0)=m^{6}\left(15731.745122+142349.56687 \varepsilon+699112.42072 \varepsilon^{2}+2468742.6339 \varepsilon^{3}+O\left(\varepsilon^{4}\right)\right)$. 


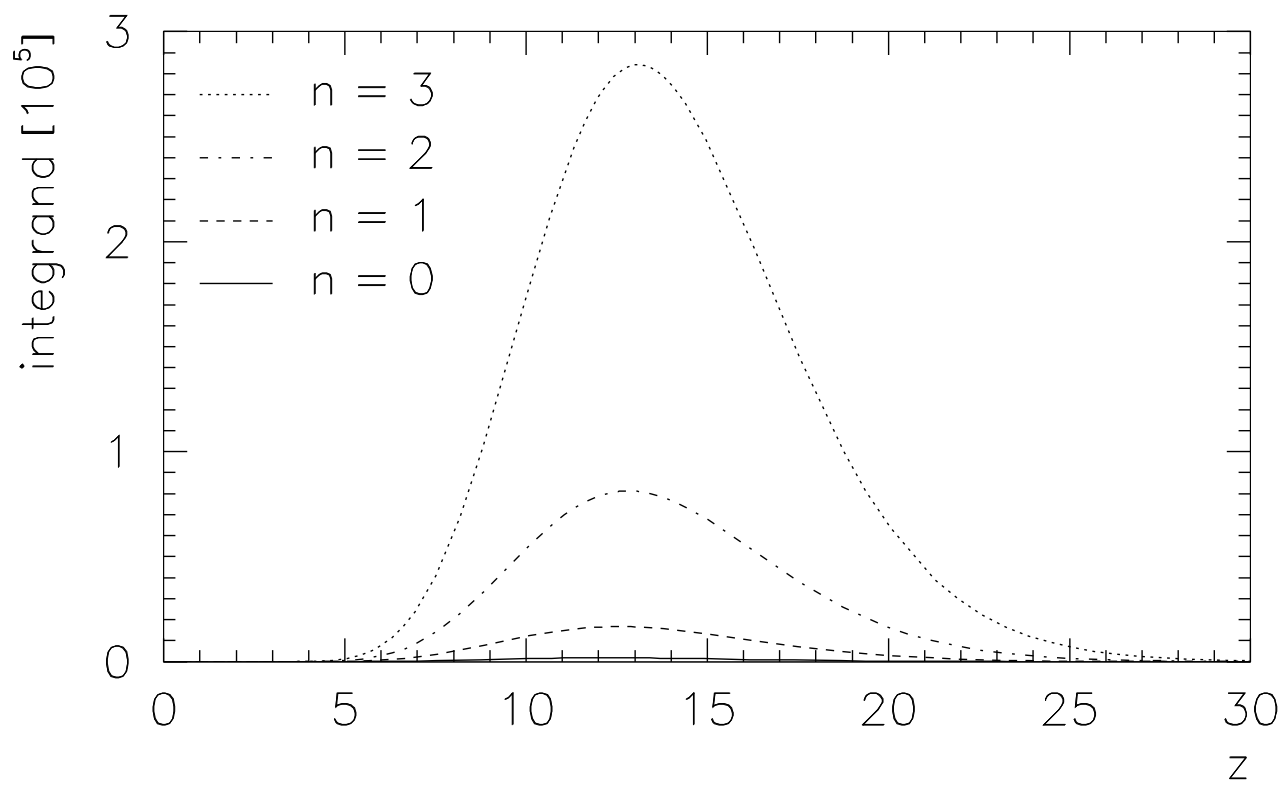

Figure 5: Integrands for numerical integration in case of the four-loop bubble at different orders $O\left(\varepsilon^{n}\right)$ in $\varepsilon$

The sum of both parts gives

$$
\begin{aligned}
\mathcal{N}_{4} \tilde{\Pi}_{4}(0)=m^{6}( & -2.5 \varepsilon^{-4}-11.6666667 \varepsilon^{-3}-31.701389 \varepsilon^{-2}-67.528935 \varepsilon^{-1} \\
& \left.-140.220621-573.53553 \varepsilon-2756.22690 \varepsilon^{2}-18239.9208 \varepsilon^{3}+O\left(\varepsilon^{4}\right)\right)
\end{aligned}
$$

which confirms the known result [137. We have not included as many digits as are given in Ref. [137].

It is not difficult to extend the analysis to higher orders in $\varepsilon$ or to a larger number of significant digits in the coefficients of the $\varepsilon$-expansion. However, since the technique is rather straightforward and simple we do not consider it worthwhile to extend the calculations into these directions. If the need arises, the potential user can tailor and optimize his or her programming code to obtain any desired accuracy and/or order of the $\varepsilon$-expansion. In our evaluation we have used standard tools provided by Wolfram's Mathematica system for symbolic manipulations which allows one to reliably control the accuracy of numerical calculations. Even at this early stage of improvement it is obvious that our algorithm is extremely simple and efficient.

\section{8 -expansion of the five-loop vacuum bubble}

In this paragraph we present results for the next-order sunrise-type diagram with $p^{2}=0$, the five-loop vacuum bubble (see Fig. (6). We have chosen to extend our calculation to the five- 


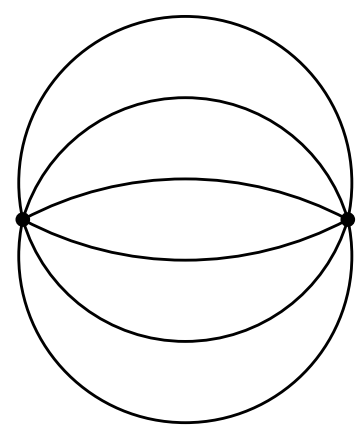

Figure 6: A five loop vacuum bubble

loop case since there exist no results on the five-loop bubble in the literature. The integral representation of the five-loop bubble with equal masses $m$ is given by

$$
\tilde{\Pi}_{5}\left(p^{2}=0\right)=\int D(x, m)^{6} d^{D} x
$$

Evaluating numerically the analytical part one obtains

$$
\begin{gathered}
\mathcal{N}_{5} \tilde{\Pi}_{5}^{\text {ana }}(0)=m^{8}\left(3 \varepsilon^{-5}+16.5 \varepsilon^{-4}+51.95833 \varepsilon^{-3}+125.6715 \varepsilon^{-2}+259.9876 \varepsilon^{-1}\right. \\
-1360392.5934-16888723.177 \varepsilon-111392297.46 \varepsilon^{2} \\
\left.-518606741.1 \varepsilon^{3}+O\left(\varepsilon^{4}\right)\right)
\end{gathered}
$$

while the numerical integration of the nonsingular part gives

$$
\begin{aligned}
\mathcal{N}_{5} \tilde{\Pi}_{5}^{\text {num }}(0)=m^{8}( & 1360739.9485+16886269.683 \varepsilon \\
& \left.+111360751.91 \varepsilon^{2}+518295438.0 \varepsilon^{3}+O\left(\varepsilon^{4}\right)\right) .
\end{aligned}
$$

The sum of both contributions is given by

$$
\begin{array}{r}
\mathcal{N}_{5} \tilde{\Pi}_{5}(0)=m^{8}\left(3 \varepsilon^{-5}+16.5 \varepsilon^{-4}+51.95833 \varepsilon^{-3}+125.6715 \varepsilon^{-2}+259.9876 \varepsilon^{-1}\right. \\
\left.+347.3551-2453.494 \varepsilon-31545.55 \varepsilon^{2}-311303.1 \varepsilon^{3}+O\left(\varepsilon^{4}\right)\right)
\end{array}
$$

One observes huge cancellation effects between the terms obtained by the analytical calculation and the numerical integration. Apparently the subtraction procedure chosen here is non-optimal. As mentioned before, the subtraction procedure should really be optimized for any given problem in order to avoid a necessity to retain high numerical precision at intermediate steps of the calculation. Nevertheless, our non-optimized simple subtraction procedure already works quite reliably with available standard computational tools. 


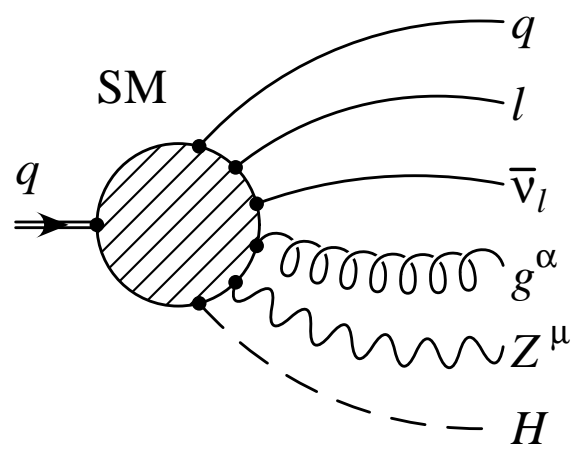

Figure 7: Example of a multi-body decay of a quark in the Standard Model

\subsection{The spectral density, the discontinuity across the physical cut and the evaluation of the multi-particle phase space}

Finally, we discuss the spectral density which can also be interpreted as the phase space volume of a multi-body decay of a single particle. An example of such a multi-body decay is shown in Fig. 7. At a given value of $s$ the value of the spectral density gives the phase space volume of the system of particles corresponding to the lines of the sunrise diagram. The phase space volume for $(n+1)$ particles with masses $m_{1}, \ldots, m_{n+1}$ is computed according to 142 ]

$$
\Phi_{n+1}\left(p ; p_{1}, \ldots, p_{n+1}\right)=\int \frac{d^{3} p_{1}}{(2 \pi)^{3} E_{1}} \ldots \frac{d^{3} p_{n+1}}{(2 \pi)^{3} 2 E_{n+1}} \delta^{(4)}\left(p_{1}+\ldots+p_{n+1}-p\right)
$$

where $E_{i}=\sqrt{\vec{p}_{i}^{2}+m_{i}^{2}}$. The optical theorem relates the imaginary part of a Feynman amplitude to on-shell propagation of its internal lines and therefore to the product of an outer state with its conjungate via so-called cutting rules 143 . (for a recent review see also Ref. [144]). Therefore the discontinuity across a physical cut of the sunrise-type diagram with $(n+1)$ internal lines carrying $(n+1)$ masses $m_{1}, \ldots, m_{n+1}$ leads to the $(n+1)$ particle phase space in a natural way. We obtain

$$
\Phi_{n+1}\left(p ; p_{1}, \ldots, p_{n+1}\right)=\left.2 \pi \rho(s)\right|_{p^{2}=s}
$$

which means that, up to a constant factor, the phase space is given by the spectral density. 


\section{Asymptotic analysis of sunrise-type diagrams}

In multi-loop calculations it is very useful to use expansions in different regimes of its kinematical variables and masses, corresponding each to the smallness of one of the parameters involved in the problem. After defining an appropriate scale, the separation of scales can considerably simplify the description in the given region of energy or momentum transfer for a particular physical process. Such an analysis is needed when, starting from an underlying full theory, one wants to formulate an effective theory for a given limited energy scale. Taking for instance Quantum Chromodynamics (QCD) as the underlying theory, the best-known of such effective theories is the Heavy Quark Effective Theory (HQET) which analyzes the behaviour of a heavy quark near its mass shell which leads to a simplified description of physical processes subject to this approximation [145]. The effective theory is directly constructed through an expansion of the expression originally written in QCD. Its analysis, therefore, relies on the expansion of Feynman diagrams in a special regime. Another important example is the expansion near the production threshold, leading to an effective theory termed nonrelativistic QCD (NRQCD). NRQCD is a more complicated case of an effective theory since one introduces new variables in NRQCD which differ from those defined in QCD. The most involved example is Chiral Perturbation Theory (ChPT) where the variables are Goldstone modes which are not directly related to QCD (and where it is still difficult to construct a direct correspondence). Nevertheless, the common thread of all these effective theories is that they use perturbative expansions in terms of diagrams in given specific kinematical regimes.

When sunrise-type diagrams enter these analyses, they usually provide the leading order contribution or serve as laboratory for checking more elaborate techniques. In this connection the expansion of sunrise-type diagrams in configuration space happens to be very efficient for many different regimes. Having the closed form expression in Eq. (18) for the correlation function $\tilde{\Pi}(p)$ at hand, different asymptotic regimes in the parameters (masses, external momentum) can easily be analyzed by calculating the corresponding expansions. The first three subsections in this section will deal with examples in the Euclidean domain. Starting with the fourth subsection, we will consider some limiting cases in the Minkowskian domain which is the domain of physical states. The calculation of asymptotic cases is more involved in this case. The calculations are performed for the spectrum given by Eq. (24) which contains the physical content of the problem. 


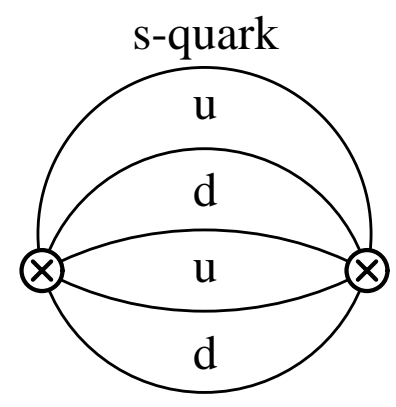

Figure 8: Leading order contribution to the two-point correlator in the sum rule analysis of light pentaquark states

\subsection{Large momentum expansion: close to the massless limit}

Loop integrations considerably simplify for massless internal lines. This holds true for the momentum space representation as well as for the configuration space representation of the loops. In some cases particle mass corrections to the massless approximation can become numerically important. For instance, in QCD the analysis of hadronic processes involving the strange quark require some special care since the mass of the $s$-quark is not small compared to the masses of the lightest quarks. On the other hand, the $s$-quark mass is still much smaller than the QCD scale $\Lambda_{\mathrm{QCD}}$. The expansion in the ratio of the $s$-quark mass to the relevant scale of the considered process is therefore very effective. This expansion is especially fruitful for the analysis of tau lepton decays [146, 147, 148].

Mass corrections to the large $p^{2}$ behaviour in the Euclidean domain (i.e. expansions in $m_{i}^{2} / p^{2}$ ) are obtained by expanding the massive propagators under the integration sign in Eq. (18) in terms of the masses $m_{i}$. The final integration is performed by using the identity

$$
\int_{0}^{\infty} x^{\mu} J_{\lambda}(p x) d x=2^{\mu} p^{-\mu-1} \frac{\Gamma((\lambda+\mu+1) / 2)}{\Gamma((\lambda-\mu+1) / 2)} .
$$

Note that all these manipulations are straightforward and can be easily implemented in a system of symbolic computations. Some care is necessary, however, when poles of the $\Gamma$-function are encountered which reflect the presence of artificial infrared singularities. The framework for dealing with such problems is well-known (see e.g. Refs. [149, 150]).

A further physics application concerns the study of pentaquark properties within the QCD sum rule approach. In Fig. 8 we show the leading order contribution to the relevant two-point correlator of the interpolating pentaquark currents which contains one massive $s$-quark line. One therefore has to determine the mass corrections to the two-point correlator. Another application of the proposed techniques concerns the analysis of certain scalar mesons which can 
be viewed as four-quark states involving the $s$-quark. For instance, the scalar meson $a_{0}$ can be considered to be a $K K$ molecule built from two $K$-mesons [151]. Yet another example is the analysis of $K^{0}-\bar{K}^{0}$ mixing in the two-point function approach [152]. Note, however, that the approach based on the three-point function analysis appears to be more direct [153].

\subsection{Small momentum expansion: close to the vacuum bubble}

The basic expression in Eq. (18) is also well suited for finding the power series expansion in $p^{2}$ because the values of the polarization function and its derivatives at $p^{2}=0$ can easily be obtained. The convenience of a $p^{2}$-expansion is demonstrated by making use of the basic identity for differentiating the Bessel function (cf. Eq. (A6) ),

$$
\frac{d^{k}}{d\left(p^{2}\right)^{k}}\left(\frac{p x}{2}\right)^{-\lambda} J_{\lambda}(p x)=\left(-\frac{x^{2}}{4}\right)^{k}\left(\frac{p x}{2}\right)^{-\lambda-k} J_{\lambda+k}(p x) .
$$

Differentiation, therefore, results in an expression which has the same functional structure as the original function. This is convenient for numerical computations. Note that sufficiently high order derivatives become UV-finite. Operationally, this is obvious since the subtraction polynomial vanishes after sufficiently high derivatives are taken. However, this can also be seen explicitly from Eq. (87) where high powers of $x^{2}$ suppress the singularity of the product of propagators at small $x$. The key integral is given by

$$
\int_{0}^{\infty} x^{\mu-1} K_{\nu}(M x) d x=2^{\mu-2} M^{-\mu} \Gamma\left(\frac{\mu+\nu}{2}\right) \Gamma\left(\frac{\mu-\nu}{2}\right) .
$$

The main physical applications are given by calculations within ChPT and HQET. The technique is especially useful for the analysis of form factors. Another application is given by expansions of heavy quark correlators through the conformal mapping in order to find an approximate spectral density from the calculation of moments [154, 155, 156]. Finally, applications can be found in the analysis of some specific rules for massive exotic states by calculating the moments of the spectral density.

\subsection{Dominating mass expansion: near the static limit}

Consider a hadron with one large mass which is of the same order as the external momentum and all other masses are small. An example is a heavy meson consisting of the c-quark and light quark(s). In the Euclidean domain the polarization function is not difficult to compute in this limit because of the simplicity of the configuration space representation and the high speed

of convergence of the ensuing numerical procedures. One can do even better since this special 
limit can be done analytically. When expanding the propagators in the limit of small masses one encounters powers of $x$ and $\ln (m x)$ (or, within the framework of dimensional regularization, non-integer powers of $x$ ). The remaining functions are the weight function (Bessel function of the first kind) and the propagator of the heavy particle with the large mass $M$ which is given by the modified Bessel function of the second kind. The general structure of the terms in the series expansion that contribute to $\tilde{\Pi}(p)$ is given by

$$
2 \pi^{\lambda+1} \int_{0}^{\infty}\left(\frac{p x}{2}\right)^{-\lambda} J_{\lambda}(p x) K_{\nu}(M x) x^{2 \rho} d x
$$

The integrations in Eq. (89) can be done in closed form by using the basic integral representation

$$
\begin{aligned}
\int_{0}^{\infty} & x^{\mu} J_{\lambda}(p x) K_{\nu}(M x) d x \\
= & \frac{p^{\lambda} \Gamma((\lambda+\mu+\nu+1) / 2) \Gamma((\lambda+\mu-\nu+1) / 2)}{2^{1-\mu} M^{\lambda+\mu+1} \Gamma(\lambda+1)} \\
& \quad \times{ }_{2} F_{1}\left((\lambda+\mu+\nu+1) / 2,(\lambda+\mu-\nu+1) / 2 ; \lambda+1 ;-p^{2} / M^{2}\right)
\end{aligned}
$$

where ${ }_{2} F_{1}(a, b ; c ; z)$ is the hypergeometric function [135]. The corresponding integrals contain integer powers of logarithms. They can be computed by differentiation with respect to $\mu$. Note that the maximal power of the logarithm is determined by the number of light propagators and does not increase with the order of the expansion: any light propagator contains only one power of the logarithm, as can be seen from the expansion of the Bessel function $K_{\nu}(\xi)$ at small $\xi[135]$.

Physical applications in this regime are mainly sum rules for hadrons including a heavy flavour [157]. These are the sum rules for the $\Lambda_{c}$ or $\Lambda_{b}$ baryons where the lowest order contribution to the sum rules are given by the genuine two-loop sunrise-type diagram. In the case of the $D$ and $B$ mesons only the one-loop (degenerate sunrise) diagram contributes.

\subsection{Threshold expansion: close to nonrelativistic physics}

The threshold region is very important for the description of heavy quarkonia states. In the threshold region the necessary calculations considerably simplify if one uses an appropriate effective theory. This effective theory is designed to remove the degrees of freedom that can be treated perturbatively from the outset and considers only the dynamics of the essential modes relevant for the considered energy scales. For heavy quarkonia near the threshold the effective theory is constructed on the basis of the non-relativistic approximation for the strong interaction and is called nonrelativistic QCD (NRQCD). Due to the use of NRQCD there has 
been a great advance in describing the heavy flavour production near threshold during the last years 158, 159, 160, 161, 162, 163, 164, 165, 166, 167.

The threshold region of a sunrise-type diagram is determined by the condition $q^{2}+M^{2} \simeq 0$ where $q$ is the Euclidean momentum and $M=\sum_{i} m_{i}$ is the threshold value for the spectral density. We introduce the Minkowskian momentum $p$ defined by $p^{2}=-q^{2}$ which allows for an analytic continuation to the physical cut. Operationally this analytic continuation can be performed by replacing $q \rightarrow i p$. To analyze the region near threshold we use the parameter $\Delta=M-p$ which can take complex values. The parameter $\Delta$ is more convenient in the Euclidean domain while the parameter $E=-\Delta=p-M$ is the actual energy counted from threshold and is used in phenomenological applications. The analytic continuation of the Fourier transform to the Minkowskian domain has the form

$$
\tilde{\Pi}(p)=2 \pi^{\lambda+1} \int_{0}^{\infty}\left(\frac{i p x}{2}\right)^{-\lambda} J_{\lambda}(i p x) \Pi(x) x^{2 \lambda+1} d x .
$$

For the threshold expansion we have to analyze the large $x$ behaviour of the integrand, corresponding to the region which saturates the integral in the limit $p \rightarrow M$ or, equivalently, $E \rightarrow 0$. It is convenient to perform the analysis in a basis where the integrand has a simple large $x$ behaviour. The most important part of the integrand is the Bessel function $J_{\lambda}(i p x)$ which, however, contains both rising and falling branches at large $x$. This resembles the situation with the elementary trigonometric functions $\sin (z)$ and $\cos (z)$ to which the Bessel function $J_{\lambda}(z)$ is rather close in a certain sense. Indeed, the function $\cos (z)$ and $\sin (z)$ are linear combinations of exponentials, namely

$$
\cos (z)=\frac{1}{2}\left(e^{i z}+e^{-i z}\right)
$$

and has also both rising and falling branches at large pure imaginary arguments: the exponentials show simple asymptotic behaviour $e^{ \pm z}$ at $z= \pm i \infty$. The analogous statement is true for $J_{\lambda}(z)$ which can be written as a sum of two Hankel functions,

$$
J_{\lambda}(z)=\frac{1}{2}\left(H_{\lambda}^{+}(z)+H_{\lambda}^{-}(z)\right)
$$

where $H_{\lambda}^{ \pm}(z)=J_{\lambda}(z) \pm i Y_{\lambda}(z)$. The Hankel functions $H_{\lambda}^{ \pm}(z)$ show a simple asymptotic behaviour at infinity,

$$
H_{\lambda}^{ \pm}(i z) \sim z^{-1 / 2} e^{ \pm z}
$$

(cf. Eq. (A16) ). Accordingly we split up $\tilde{\Pi}(p)$ into $\tilde{\Pi}(p)=\tilde{\Pi}^{+}(p)+\tilde{\Pi}^{-}(p)$ with

$$
\tilde{\Pi}^{ \pm}(p)=\pi^{\lambda+1} \int_{0}^{\infty}\left(\frac{i p x}{2}\right)^{-\lambda} H_{\lambda}^{ \pm}(i p x) \Pi(x) x^{2 \lambda+1} d x .
$$




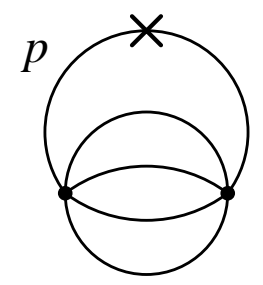

Figure 9: Representation of $\tilde{\Pi}^{+}(p)$ as a standard vacuum bubble with an additional line. The cross on the line denotes an arbitrary number of derivatives of that line.

The two parts $\tilde{\Pi}^{ \pm}(p)$ of the polarization function $\tilde{\Pi}(p)$ have a completely different behaviour near threshold which allows one to analyze them independently. This observation makes the subsequent analysis straightforward.

We first consider the contribution of the $\tilde{\Pi}^{+}(p)$ part which reduces to a regular sunrise-type diagram. Indeed, using the relation given by Eq. (A14),

$$
K_{\lambda}(z)=\frac{\pi i}{2} e^{i \lambda \pi / 2} H_{\lambda}^{+}(i z)
$$

between Bessel functions of different kinds one can replace the Hankel function $H_{\lambda}^{+}(i p x)$ with the Bessel function $K_{\lambda}(p x)$. Since the propagator of a massive particle (massive line in the diagram) is given by the Bessel function up to a power in $x$, this substitution shows that the weight function behaves like a propagator of an additional line with the "mass" $p$. The explicit expression is given by

$$
\tilde{\Pi}^{+}(p)=\frac{(-2 \pi i)^{2 \lambda+1}}{\left(p^{2}\right)^{\lambda}} \int_{0}^{\infty} \Pi_{+}(x) x^{2 \lambda+1} d x .
$$

The function $\Pi_{+}(x)=\Pi(x) D(x, p)$ is the polarization function of a new effective diagram which is equal to the initial polarization function multiplied by a propagator with $p$ as mass parameter. We thus end up with a vacuum bubble of the sunrise-type type with one additional line compared to the initial diagram (see Fig. 9). All derivatives of $\tilde{\Pi}^{+}(p) \equiv \tilde{\Pi}^{+}(M-\Delta)$ with respect to $\Delta$ are represented as vacuum bubbles with one additional line carrying rising indices. Such diagrams can be efficiently calculated within the recurrence relation technique developed in [37, 38, 39]. They possess no singularities at the production threshold $p=M$. This can be seen by looking at the expansion for large $x$. The behaviour at large $x$ is given by the asymptotic form of the functions for which one obtains

$$
H^{+}(i p x)=\sqrt{\frac{2}{i \pi p x}} e^{-p x}\left(1+O\left(x^{-1}\right)\right), \quad K(m x)=\sqrt{\frac{\pi}{2 m x}} e^{-m x}\left(1+O\left(x^{-1}\right)\right)
$$

(cf. Eqs. (A15) and (A16)). The large $x$ range of the integral (above a reasonably large cutoff 
parameter $\Lambda$ ) has the general form

$$
\tilde{\Pi}_{\Lambda}^{+}(M-\Delta) \sim \int_{\Lambda}^{\infty} x^{-a} e^{-(2 M-\Delta) x} d x
$$

where

$$
a=(n-1)(\lambda+1 / 2) .
$$

The right hand side of Eq. (99) is an analytic function in $\Delta$ in the vicinity of $\Delta=0$. It exhibits no cut or other singularities near the threshold and therefore does not contribute to the spectral density.

In contrast to the previous case, the integrand of the second part $\tilde{\Pi}^{-}(p)$ contains $H^{-}(i p x)$ which behaves like a rising exponential function at large $x$,

$$
H^{-}(i p x) \sim x^{-1 / 2} e^{p x}
$$

(cf. Eq. (A16). Therefore, the integral is represented by

$$
\tilde{\Pi}_{\Lambda}^{-}(M-\Delta) \sim \int_{\Lambda}^{\infty} x^{-a} e^{-\Delta x} d x
$$

The function $\tilde{\Pi}^{-}(M-\Delta)$ is non-analytic near $\Delta=0$ because for $\Delta<0$ the integrand in Eq. (102) grows in the large $x$ region and the integral diverges at the upper limit. Therefore the function which is determined by this integral is singular at $\Delta<0(E>0)$ and requires an interpretation for these values of the argument $\Delta$. The function is analytic in the complex $\Delta$-plane with a cut along the negative axis. This cut corresponds to the physical positive energy cut. The discontinuity across the cut gives rise to the non-vanishing spectral density of the diagram (cf. Appendix C).

The integral for $\tilde{\Pi}^{-}(p)$ in Eq. (95) cannot be done analytically. In order to obtain an expansion for the spectral density near the threshold in analytical form we make use of the asymptotic series expansion for the function $\Pi(x)$ which crucially simplifies the integrands but still preserves the singular structure of the integral in terms of the variable $\Delta$. The asymptotic series expansion of the main part of each propagator, i.e. of the modified Bessel function of the second kind, to the order $N$ is given by Eq. (A15). The asymptotic expansion of the function $\Pi(x)$ therefore consists of an exponential factor $e^{-M x}$ and an inverse power series in $x$ up to the order $\tilde{N}$, where $\tilde{N}$ is closely related to $N$. It is this asymptotic expansion that determines the singularity structure of the integral. We write the whole integral in the form of a sum of two terms,

$$
\begin{aligned}
\tilde{\Pi}^{-}(p)= & \pi^{\lambda+1} \int\left(\frac{i p x}{2}\right)^{-\lambda} H_{\lambda}^{-}(i p x)\left(\Pi(x)-\Pi_{N}^{a s}(x)\right) x^{2 \lambda+1+2 \varepsilon} d x \\
& +\pi^{\lambda+1} \int\left(\frac{i p x}{2}\right)^{-\lambda} H_{\lambda}^{-}(i p x) \Pi_{N}^{a s}(x) x^{2 \lambda+1+2 \varepsilon} d x=\tilde{\Pi}^{d i}(p)+\tilde{\Pi}^{a s}(p) .
\end{aligned}
$$


The integrand of the first term $\tilde{\Pi}^{d i}(p)$ behaves as $1 / x^{\tilde{N}}$ at large $x$ while the integrand of the second term accumulates all lower powers of the large $x$ expansion. Note that only the large $x$ behaviour is essential for the near threshold expansion of the spectral density. This fact has been taken into account in Eqs. (99) and (102) where we introduced a cutoff $\Lambda$. However, from the practical point of view the calculation of the regularized integrals with an explicit cutoff is inconvenient. The final result of the calculation - the spectral density of the diagram - is independent of the cutoff, but the integration is technically complicated if the cutoff is introduced. However, in extending the integration over the whole region of the variable $x$ without using the cutoff one immediately encounters divergences at small $x$ because the asymptotic expansion is invalid in the region near the origin, so one is not allowed to continue it to this region. The standard way to cope with such a situation is to introduce dimensional regularization. It allows one to deal with divergent expressions at intermediate stages of the calculation and is technically simple because it does not introduce any cutoff and therefore does not modify the integration region very much. Note that dimensional regularization does not necessarily regularize all divergences in this case (in contrast to the standard case of ultraviolet divergences) but it nevertheless suffices for our purposes. We shall use a parameter $\varepsilon$ to regularize the divergences at small $x$.

The first part $\tilde{\Pi}^{d i}(p)$ in Eq. (103) containing a difference of the polarization function and its asymptotic expansion since the integrand gives no contributions to the spectral density up to a given order of the expansion in $\Delta$. This is so because the subtracted asymptotic series to order $N$ cancels the inverse power behaviour of the integrand to this degree $N$. The integrand decreases sufficiently fast for large values of $x$ and the integral converges even at $\Delta=0$. Therefore this term is inessential for the evaluation of the expansion of the spectral density up to given order.

The expansion of the spectral density at small $E$ is determined only by the integral $\tilde{\Pi}^{a s}(p)$ in Eq. (103). This integral is still rather complicated to compute but we can go a step further in its analytical evaluation. Indeed, since the singular behaviour of $\tilde{\Pi}^{a s}(p)$ is determined by the behaviour at large $x$, we can replace the first factor, i.e. the Hankel function, in the large $x$ region by its asymptotic expansion up to some order $N$. We use Eq. (A16) to obtain a representation

$$
\tilde{\Pi}^{d a s}(p)=\pi^{\lambda+1} \int\left(\frac{i p x}{2}\right)^{-\lambda} H_{\lambda, N}^{-a s}(i p x) \Pi_{N}^{a s}(x) x^{2 \lambda+1+2 \varepsilon} d x .
$$

The index "das" stands for "double asymptotic" and indicates that the integrand in Eq. (104) consists of a product of two asymptotic expansions: one for the polarization function $\Pi(x)$ and another for the Hankel function $H_{\lambda}(x)$ as weight (or kernel). Both asymptotic expansions 
are straightforward and can be obtained from standard handbooks on Bessel functions (cf. Appendix (A). We therefore arrive at our final result: the integration necessary for evaluating the near threshold expansion of the sunrise-type diagrams reduces to integrals of Euler's Gamma function type, i.e. integrals containing exponentials and powers. Indeed, the result of the expansion in Eq. (104) is an exponential function $e^{-\Delta x}$ times a power series in $1 / x$, namely

$$
x^{-a+2 \varepsilon} e^{-\Delta x} \sum_{j=0}^{N-1} \frac{A_{j}}{x^{j}}
$$

where $a$ has already been defined in Eq. (100) and the coefficients $A_{j}$ are simple functions of the momentum $p$ and the masses $m_{i}$. The expression in Eq. (105) can be integrated analytically using

$$
\int_{0}^{\infty} x^{-a+2 \varepsilon} e^{-\Delta x} d x=\Gamma(1-a+2 \varepsilon) \Delta^{a-1-2 \varepsilon}
$$

The result is

$$
\tilde{\Pi}^{d a s}(M-\Delta)=\sum_{j=0}^{N-1} A_{j} \Gamma(1-a-j+2 \varepsilon) \Delta^{a+j-1-2 \varepsilon} .
$$

This expression is our final representation for the part of the polarization function of a sunrisetype diagram necessary for the calculation of the spectral density near the production threshold (see Appendix (C). The spectral density is a function of $E=-\Delta$ and will be denoted by $\tilde{\rho}(E)=\rho\left((M+E)^{2}\right)$ in the following. Starting from the main result in Eq. (107), we discuss the general structure in detail. In the case where $a$ takes integer values, these coefficients result in $1 / \varepsilon$-divergences for small values of $\varepsilon$. The powers of $\Delta$ in Eq. (107) have to be expanded to first order in $\varepsilon$ and give

$$
\frac{1}{2 \varepsilon} \Delta^{2 \varepsilon}=\frac{1}{2 \varepsilon}+\ln \Delta+O(\varepsilon)
$$

Because of

$$
\operatorname{Disc} \ln (\Delta) \equiv \ln (-E-i 0)-\ln (-E+i 0)=-2 \pi i \theta(E)
$$

$\tilde{\Pi}^{\text {das }}(M-\Delta)$ in Eq. (107) contributes to the spectral density. For half-integer values of $a$ the power of $\Delta$ itself has a cut even for $\varepsilon=0$. The discontinuity is then given by

$$
\operatorname{Disc} \sqrt{\Delta}=-2 i \sqrt{E} \theta(E)
$$

Our method to construct a threshold expansion thus simply reduces to the analytical calculation of the integral in Eq. (104) which can be done for arbitrary dimension and an arbitrary number of lines with different masses. In the next paragraphs we use our technique to work out some specific examples which demonstrate both the simplicity and efficiency of our method. 


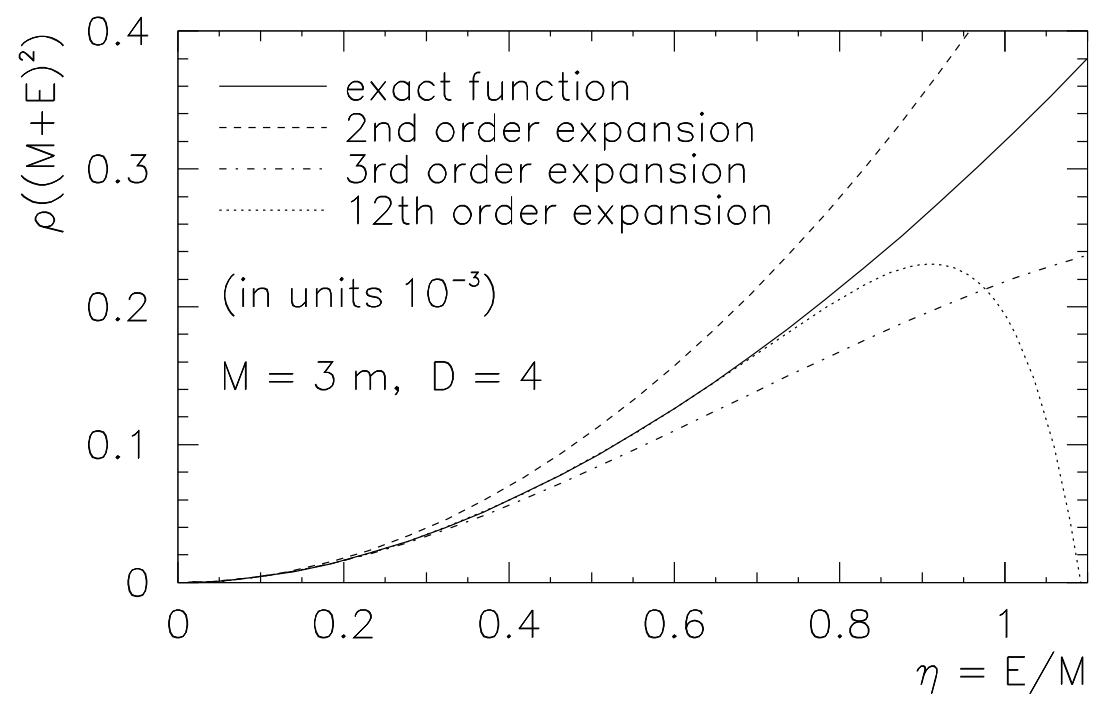

Figure 10: Various results for the spectral density for $n=3$ equal masses in $D=4$ spacetime dimensions as function of the threshold parameter $E / M$. Shown are the exact solution obtained by using Eq. (112) (solid curve) and threshold expansions for different orders taken from Eq. (114) (dashed to dotted curves).

\subsection{Equal mass genuine sunrise diagram at threshold}

The polarization function corresponding to the genuine sunrise diagram with three propagators with equal masses $m$ in $D=4$ space-time dimensions is given by

$$
\Pi(x)=\frac{m^{3} K_{1}(m x)^{3}}{(2 \pi)^{6} x^{3}} .
$$

The exact spectral density is given by the integral representation in Eq. (24) which for this particular case reads

$$
\rho(s)=\frac{2 \pi}{i \sqrt{s}} \int_{c-i \infty}^{c+i \infty} I_{1}(x \sqrt{s}) \Pi(x) x^{2} d x .
$$

In order to obtain a threshold expansion of the spectral density in Eq. (112) we use Eq. (107) to calculate the expansion of the appropriate part of the polarization function. To illustrate the procedure we derive the explicit functional behaviour of the integrand in Eq. (104) which is given by an asymptotic expansion at large $x$,

$$
\begin{aligned}
\pi^{2}\left(\frac{i p x}{2}\right)^{-1} & H_{1, N}^{a s}(p x) \Pi_{N}^{a s}(x) x^{3+2 \varepsilon}=\frac{m^{3 / 2} e^{(p-3 m) x}}{(4 \pi)^{3} p^{3 / 2}} x^{-3+2 \varepsilon} \times \\
\times & \left\{1+\frac{9}{8 m x}-\frac{3}{8 p x}+\frac{9}{128 m^{2} x^{2}}-\frac{27}{64 m p x^{2}}-\frac{15}{128 p^{2} x^{2}}+O\left(x^{-3}\right)\right\} .
\end{aligned}
$$


From Eq. (113) we can easily read off the coefficients $A_{j}$ that enter the expansion in Eq. (105). The spectral density is obtained by performing a term-by-term integration of the series in Eq. (113) and by evaluating the discontinuity across the cut along the positive energy axis $E>0$. The result reads

$$
\begin{aligned}
\tilde{\rho}(E)= & \frac{E^{2}}{384 \pi^{3} \sqrt{3}}\left\{1-\frac{1}{2} \eta+\frac{7}{16} \eta^{2}-\frac{3}{8} \eta^{3}+\frac{39}{128} \eta^{4}-\frac{57}{256} \eta^{5}\right. \\
& \left.+\frac{129}{1024} \eta^{6}-\frac{3}{256} \eta^{7}-\frac{4047}{32768} \eta^{8}+\frac{18603}{65536} \eta^{9}-\frac{248829}{524288} \eta^{10}+O\left(\eta^{11}\right)\right\}
\end{aligned}
$$

where the notation $\eta=E / M, M=3 m$ is used. The simplicity of the derivation is striking. By no cost it can be generalized to any number of lines, arbitrary masses, and any space-time dimension. The genuine equal mass sunrise has been chosen for definiteness only. It also allows us to compare our results with results available in the literature. Eq. (114) reproduces the expansion coefficients $\tilde{a}_{j}$ obtained in Ref. [168] (the fourth column in Table 1 of Ref. 168]) by a direct integration in momentum space within the technique of region separation [169]. In Fig. 10 the exact solution is shown together with expansions to various orders.

\subsection{Three loops and a route to any number of loops at threshold}

The sunrise-type diagrams with four or more propagators cannot be easily done by using the momentum space technique because it requires the multi-loop integration of entangled momenta. As emphasized before the configuration space technique allows one to immediately generalize our previous results to any number of lines (or loops) with no additional effort. Consider first the three-loop case of sunrise-type diagrams. The polarization function of the equal mass sunrise-type diagram with four propagators in $D=4$ space-time is given by

$$
\Pi(x)=\frac{m^{4} K_{1}(m x)^{4}}{(2 \pi)^{8} x^{4}} .
$$

The exact spectral density of this diagram can be obtained from Eq. (112) while the near threshold expansion can be found using Eq. (107). The expansion of the spectral density near threshold reads

$$
\begin{aligned}
& \tilde{\rho}(E)=\frac{E^{7 / 2} M^{1 / 2}}{26880 \pi^{5} \sqrt{2}}\left\{1-\frac{1}{4} \eta+\frac{81}{352} \eta^{2}-\frac{2811}{18304} \eta^{3}+\frac{17581}{292864} \eta^{4}\right. \\
& \left.+\frac{1085791}{19914752} \eta^{5}-\frac{597243189}{3027042304} \eta^{6}+\frac{4581732455}{12108169216} \eta^{7}-\frac{496039631453}{810146594816} \eta^{8}+O\left(\eta^{9}\right)\right\}
\end{aligned}
$$

where $\eta=E / M$ and $M=4 m$ is the threshold value. One sees the difference with the previous three-line case. In Eq. (116) the cut corresponds to a square root branch cut while in the 
three-line case one has a logarithmic cut. One can easily figure out the reason for this by looking at the asymptotic structure of the integrand. For an even number of lines (i.e. odd number of loops) one has a square root branch cut, while for an odd number of lines (even number of loops) one has a logarithmic branch cut. This statement generalizes from $D=4$ to any even space-time dimension. In the general case the structure of the cut depends on the dimensionality of the space-time. The general formula for the $n$-loop case reads

$$
\tilde{\rho}(E) \sim E^{(\lambda+1 / 2) n-1}(1+O(E))
$$

For $D=4$ space-time dimension (i.e. $\lambda=1$ ) we can verify the result of Ref. [37, 38, 39,

$$
\tilde{\rho}(E) \sim E^{(3 n-2) / 2}(1+O(E))
$$

Returning to Eq. (116) one has numerically

$$
\begin{aligned}
& \tilde{\rho}(E)=8.5962 \cdot 10^{-5} E^{7 / 2} M^{1 / 2}\left\{1.000-0.250 \eta+0.230 \eta^{2}\right. \\
& \left.\quad-0.154 \eta^{3}+0.060 \eta^{4}+0.055 \eta^{5}-0.197 \eta^{6}+0.378 \eta^{7}-0.612 \eta^{8}+O\left(\eta^{9}\right)\right\}
\end{aligned}
$$

where we have written down the coefficients up to three decimal places. It is difficult to say anything definite about the convergence of this series. By construction it is an asymptotic series. However, we stress that the practical (or explicit) convergence can always be checked by comparing series expansions like the one shown in Eq. (119) with the exact spectral density given in Eq. (112) by numerical integration.

We conclude that the spectral density of the sunrise-type diagram can be efficiently calculated within the configuration space technique. It does not matter whether one is aiming for the exact result or an expansion the configuration space technique which can readily deliver the desired result. The exact formula in Eq. (112) as well as the threshold expansion obtained from it can be used to calculate the spectral density for an arbitrarily large number of internal lines. We stress that the case of different masses does not lead to any complications within the configuration space technique: the exact formula in Eq. (24) and/or the near threshold expansion work equally well for any configuration of masses. We do not present plots for general cases of different masses because they are not very illuminating as one can only see the common threshold. However, there is an interesting kinematic regime for the different mass case which is important for certain applications which, to the best of our knowledge, has not been treated in the literature before. An analytical solution for the expansion of the spectral density in this regime is given in the next subsection. 


\subsection{New features of the threshold expansion: resummation of small mass effects for strongly asymmetric mass configurations}

The main question in constructing expansions is their practical usefulness and the region of validity. The threshold expansion for equal or almost equal masses breaks down for instance for $E \approx M=\sum m_{i}$. However, if the masses are not equal, the region of the breakdown of the expansion is determined by the mass with the smallest numerical value. The simplest example where one can see this phenomenon is the analytical expression for the spectral density of the simple loop (degenerate sunrise-type diagram) with two different masses $m_{1}$ and $m_{2}$. In $D=4$ space-time dimensions (see e.g. Ref. [37, 38, 39]) one has

$$
\tilde{\rho}(E)=\frac{\sqrt{E\left(E+2 m_{1}\right)\left(E+2 m_{2}\right)(E+2 M)}}{(4 \pi(M+E))^{2}}
$$

where $M=m_{1}+m_{2}$. The threshold expansion is obtained by expanding the right hand side of Eq. (120) in $E$ for small values of $E$. If $m_{2}$ is much smaller than $m_{1}$, the expansion breaks down at $E \approx 2 m_{2}$. The break-down of the series expansion can also be observed in more general cases. If one of the masses (which we call $m_{0}$ ) is much smaller than the other masses, the threshold expansion is only valid in a very limited region $E \lesssim 2 m_{0}$.

To generalize the expansion and extend it to the region of $E \sim M$ one has to treat the smallest mass exactly. In this case one can use a method which we call the resummation of small mass effects [40]. This method will be explained in the following. We start with the representation

$$
\tilde{\Pi}^{p a s}(p)=\pi^{\lambda+1} \int\left(\frac{i p x}{2}\right)^{-\lambda} H_{\lambda, N}^{-a s}(i p x) \Pi_{m_{0}}^{a s}(x) x^{2 \lambda+1+2 \varepsilon} d x
$$

which is the part of the polarization function contributing to the spectral density. The integrand in Eq. (121) has the form

$$
\Pi_{m_{0}}^{a s}(x)=\Pi_{n-1}^{a s}(x) D\left(m_{0}, x\right)
$$

where the asymptotic expansions are substituted for all the propagators except for the one with the small mass $m_{0}$. This is indicated by the index "pas" in Eq. (121) which stands for "partial asymptotic". The main technical observation leading to the generalization of the expansion method is that $\tilde{\Pi}^{\text {pas }}(p)$ is still analytically computable in a closed form. Indeed, the genuine integral to compute has the form

$$
\begin{aligned}
\int_{0}^{\infty} & x^{\mu-1} e^{-\tilde{\alpha} x} K_{\nu}(\beta x) d x= \\
\quad= & \frac{\sqrt{\pi}(2 \beta)^{\nu}}{(2 \tilde{\alpha})^{\mu+\nu}} \frac{\Gamma(\mu+\nu) \Gamma(\mu-\nu)}{\Gamma(\mu+1 / 2)}{ }_{2} F_{1}\left(\frac{\mu+\nu}{2}, \frac{\mu+\nu+1}{2} ; \mu+\frac{1}{2} ; 1-\frac{\beta^{2}}{\tilde{\alpha}^{2}}\right)
\end{aligned}
$$


where $\tilde{\alpha}=\Delta-m_{0}$ and $\beta=m_{0}$. The integral $\tilde{\Pi}^{\text {pas }}(p)$ in Eq. (121) is thus expressible in terms of hypergeometric functions [170. For the construction of the spectral density one has to find the discontinuity of the right hand side of Eq. (123). There are several ways to do this. We proceed by applying the discontinuity operation to the integrand of the integral representation of the hypergeometric function. The resulting integrals are calculated again in terms of hypergeometric functions. Indeed,

$$
\begin{aligned}
& \frac{1}{2 \pi i} \operatorname{Disc} \int_{0}^{\infty} x^{\mu-1} e^{\alpha x} K_{\nu}(\beta x) d x= \\
& \quad=\frac{2^{\mu}\left(\alpha^{2}-\beta^{2}\right)^{1 / 2-\mu}}{\alpha^{1 / 2-\nu} \beta^{\nu}} \frac{\Gamma(3 / 2)}{\Gamma(3 / 2-\mu)}{ }_{2} F_{1}\left(\frac{1-\mu-\nu}{2}, \frac{2-\mu-\nu}{2} ; \frac{3}{2}-\mu ; 1-\frac{\beta^{2}}{\alpha^{2}}\right)
\end{aligned}
$$

where $\alpha=E+m_{0}$. The final expression in Eq. (124) completely solves the problem of the generalization of the near threshold expansion technique. For integer values of $\mu$ there are no singular Gamma functions (with negative integer argument). Therefore we abolish the regularization and set $\varepsilon=0$ when using this expression. We have thus found a direct transition from the polarization function (expressed through the integral) to the spectral density in terms of one hypergeometric function for each genuine integral. There is no need to use the recurrence relations available for hypergeometric functions.

As an example we present the (over)simplified case of the two-line diagram with masses $m$ and $m_{0} \ll m$ in four space-time dimensions. We cite this example because the expansion of the spectral density and its generalized expansion can be readily compared analytically with the exact result in Eq. (120). Both expansions, the pure and the resummed expansion, will be compared with the exact anaytical result.

The pure expansion of the spectral density near threshold (the second order asymptotic expansion should suffice to show the general features in a short and concise form) is given by

$$
\begin{aligned}
\tilde{\rho}^{d a s}(E)= & \frac{\sqrt{2 m_{0} m E}}{8 \pi^{2} M^{3 / 2}}\left\{1+\left(\frac{1}{m}+\frac{1}{m_{0}}-\frac{7}{M}\right) \frac{E}{4}\right. \\
& \left.-\left(\frac{1}{m_{0}^{2}}+\frac{1}{m^{2}}+\frac{12}{m_{0} m}-\frac{79}{M^{2}}\right) \frac{E^{2}}{32}+O\left(E^{3}\right)\right\}
\end{aligned}
$$

where $M=m+m_{0}$. As mentioned above, this series breaks down for $E>2 m_{0}$ (see Eq. (120)). The analytical expression for the spectral density of the polarization function in Eq. (121) within the generalized asymptotic expansion based on Eq. (124) is given by

$$
\begin{gathered}
\tilde{\rho}^{\text {pas }}(E)=\frac{\sqrt{m E\left(E+2 m_{0}\right)}}{8 \pi^{2}(E+M)^{3 / 2}}\left\{{ }_{2} F_{1}\left(0, \frac{1}{2} ; \frac{3}{2} ; 1-\frac{m_{0}^{2}}{\left(E+m_{0}\right)^{2}}\right)\right. \\
+\frac{E\left(E+2 m_{0}\right)}{8 m(E+M)}{ }_{2} F_{1}\left(\frac{1}{2}, 1 ; \frac{5}{2} ; 1-\frac{m_{0}^{2}}{\left(E+m_{0}\right)^{2}}\right)
\end{gathered}
$$




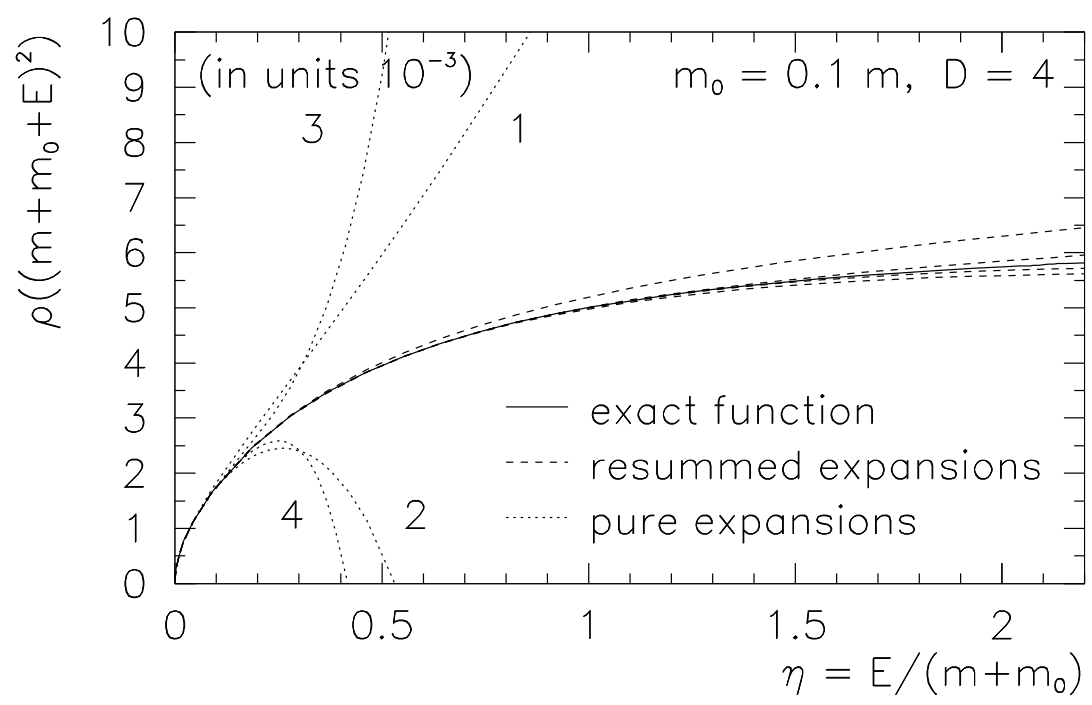

Figure 11: Various solutions for the one-loop spectral density for two different masses $m$ and $m_{0} \ll m$ in $D=4$ space-time dimensions. Shown are the exact solution which is obtained by using Eq. (112) (solid curve), the pure threshold expansions using Eq. (125) (dotted curves), and the solutions for the resummation of small mass effects such as in Eq. (126) (dashed curves). For both case the expansions are shown from the first up to the fourth order in the asymptotic expansion. For the pure threshold expansion the order is indicated explicitly.

$$
\left.-\frac{E^{2}\left(E+2 m_{0}\right)^{2}}{128 m^{2}(E+M)^{2}}\left(1+\frac{16 m(E+M)}{5\left(E+m_{0}\right)^{2}}\right){ }_{2} F_{1}\left(1, \frac{3}{2} ; \frac{7}{2} ; 1-\frac{m_{0}^{2}}{\left(E+m_{0}\right)^{2}}\right)+\ldots\right\} .
$$

We have set the regularization parameter $\varepsilon=0$ because the spectral density is finite. With $\varepsilon=0$ the resulting expressions for the hypergeometric functions in Eq. (124) simplify. The first term in the curly brackets of Eq. (126) is obviously equal to 1 in this limit because the first parameter of the hypergeometric function vanishes for $\varepsilon=0$. However, we keep Eq. (126) in its given form to show the structure of the contributions. The generalized threshold expansion has the form

$$
\tilde{\rho}^{p a s}(E)=g_{0}\left(E, m_{0}\right)+E g_{1}\left(E, m_{0}\right)+E^{2} g_{2}\left(E, m_{0}\right)+\ldots
$$

where the functions $g_{j}\left(E, m_{0}\right)$ represent effects of the resummation of small mass effects and are not polynomials in the threshold parameter $E$. In the simple one-loop case the hypergeometric functions reduce to elementary functions. For instance,

$$
{ }_{2} F_{1}\left(\frac{1}{2}, 1 ; \frac{5}{2} ; 1-\frac{m_{0}^{2}}{\left(E+m_{0}\right)^{2}}\right)=
$$




$$
=\frac{3\left(E+m_{0}\right)}{2 E\left(E+2 m_{0}\right)}\left(E+m_{0}-\frac{m_{0}^{2}}{2 \sqrt{E\left(E+2 m_{0}\right)}} \ln \left(\frac{E+m_{0}+\sqrt{E\left(E+2 m_{0}\right)}}{E+m_{0}-\sqrt{E\left(E+2 m_{0}\right)}}\right)\right) .
$$

Higher order contributions are given by hypergeometric functions with larger numerical values of the parameters. They can be simplified by using Gaussian recurrence relations for hypergeometric functions. $^{2}$

In Fig. 11 we compare the exact result of Eq. (112) with the pure expansion in Eq. (125) and the resummed expansion in Eq. (126). While the pure expansion breaks down already at the order $E \sim m_{0}$, the convergence of the expansion in Eq. (126) breaks down only at $E \sim M=m+m_{0}$. The resummation leads to an essential improvement of the convergence in comparison with the pure threshold expansion. Further examples and their discussion can be found in Ref. [40].

\subsection{Large $s$ expansion: Expansion of the spectrum at large energies}

In the Minkowskian domain where $s=p^{2}$ the situation is closely related to the large $p$ expansion if all masses are small compared to the external momentum. We start with a simple example which will be calculated in momentum space as well as in configuration space. In the next paragraph we give more involved examples.

As a first example for an expansion in $\mathrm{m}^{2} / \mathrm{s}$ we look at a case where the spectral density is given by a finite series only. For the one-loop sunrise-type diagram with one massive and one massless line we can use Feynman parametrization and obtain in (Euclidean) momentum space

$$
\tilde{\Pi}(p)=\frac{\Gamma(\varepsilon)}{(4 \pi)^{2-\varepsilon}} \int_{0}^{1}(1-x)^{-\varepsilon}\left(x p^{2}+m^{2}\right)^{-\varepsilon} d x .
$$

In order to determine the spectral desity, the discontinuity can be calculated already on the level of the integrand. A discontinuity appears if $x p^{2}+m^{2}<0$ which is satisfied for $p^{2}<-m^{2}$. We refer to Appendix $\mathbb{C}$ for more details. If we parametrize $p^{2}=s e^{i \varphi}$, we can reach the cut from both sides $\varphi=-\pi$ and $\varphi=+\pi$ and can calculate the difference, i.e. the discontinuity

$$
\operatorname{Disc}\left(m^{2}-x s\right)^{-\varepsilon}=\frac{2 \pi i}{\Gamma(\varepsilon) \Gamma(1-\varepsilon)}\left(x s-m^{2}\right)^{-\varepsilon} \theta\left(x s-m^{2}\right) \text {. }
$$

For the spectral density we obtain

$$
\rho(s)=\left.\frac{1}{2 \pi i} \operatorname{Disc} \tilde{\Pi}(p)\right|_{p^{2}=-s}=\frac{1}{(4 \pi)^{2-\varepsilon} \Gamma(1-\varepsilon)} \int_{m^{2} / s}^{1}(1-x)^{-\varepsilon}\left(x s-m^{2}\right)^{-\varepsilon} d x .
$$

\footnotetext{
${ }^{2}$ Note that Eq. (128) does not lead to the exact function in Eq. (120) because terms of order $E^{N}$ are missing which originate from the difference part $\tilde{\Pi}^{d i}(p)$ of the correlator. The difference part simply corrects the behaviour of the coefficient functions by the small mass contributions.
} 
Because the general factor is no longer singular, we can calculate the integral for $\varepsilon=0$ where the integrand is simply unity. Then we obtain

$$
\rho(s)=\frac{1}{(4 \pi)^{2}} \int_{m^{2} / s}^{1} d x=\frac{1}{(4 \pi)^{2}}\left(1-\frac{m^{2}}{s}\right) .
$$

This result can also be obtained by using configuration space techniques. At first sight one may think that this example is too simple to be worth to be calculated using configuration space techniques. Later on we shall see that for the multi-loop case the configuration space method is the only one which leads to an analytical result.

In configuration space the spectral density of the one-loop diagram with one massive and one massless line in four-dimensional spacetime is given by Eq. (112). The integral contains the modified Bessel function of the first kind, $I_{1}(z)$. Because $K_{1}(x)$ is a pure exponentially decreasing function in its asymptotic expansion while $K_{1}(x) \pm i \pi I(x)$ increases exponentially along the real axis if continued in the upper resp. lower complex plane (note the ambiguity due to Stokes' phenomenon mentioned in Appendix (C), we can divide according to

$$
\begin{aligned}
i \pi \int_{\epsilon}^{\epsilon+i \infty} I_{1}(z) f(z) d z & =\int_{\epsilon}^{\epsilon+i \infty}\left(i \pi I_{1}(z)-K_{1}(z)\right) f(z) d z+\int_{\epsilon}^{\epsilon+i \infty} K_{1}(z) d z \\
i \pi \int_{\epsilon-i \infty}^{\epsilon} I_{1}(z) f(z) d z & =\int_{\epsilon-i \infty}^{\epsilon}\left(i \pi I_{1}(z)+K_{1}(z)\right) f(z) d z-\int_{\epsilon-i \infty}^{\epsilon} K_{1}(z) d z
\end{aligned}
$$

and can lower the paths to the negative resp. positive real axis by using Cauchy's theorem realizing that the quarter circle integrals in the corresponding quadrant vanish (see Ref. [38]). The origin and the negative real axis is circumvented by two half-circle paths $C_{-}$(lower half plane) and $C_{+}$(upper half plane) in positive direction. The result found in Ref. 38] reads

$$
\begin{aligned}
& i \pi \int_{c-i \infty}^{c+i \infty} I_{1}(x \sqrt{s}) \Pi(x) x^{2} d x=\int_{\epsilon}^{\infty} K_{1}(r \sqrt{s})\left(2 \Pi(r)-\Pi\left(e^{i \pi} r\right)-\Pi\left(e^{-i \pi} r\right)\right) r^{2} d r \\
& \quad+\int_{C_{-}}\left(i \pi I_{1}(z \sqrt{s})+K_{1}(z \sqrt{s})\right) \Pi(z) z^{2} d z+\int_{C_{+}}\left(i \pi I_{1}(z \sqrt{s})-K_{1}(z \sqrt{s})\right) \Pi(z) z^{2} d z
\end{aligned}
$$

For our simple example we have to take the correlator function $\Pi(x)=D(x, m) D(x, 0)$. While the massless propagator is invariant under the multiplication of $e^{ \pm i \pi}= \pm 1$ of its argument, i.e. $D(-x, 0)=D(x, 0)$, for the massive propagator we obtain

$$
D\left(e^{ \pm i \pi} r, m\right)=\frac{(m r)}{(2 \pi)^{2} r^{2}}\left(K_{1}(m r) \pm i \pi I_{1}(m r)\right)
$$

where $K_{\lambda}\left(e^{ \pm i \pi} x\right)=e^{\mp i \pi \lambda} K_{\lambda}(x) \mp i \pi I_{\lambda}(x)$ is used. We obtain $2 \Pi(r)-\Pi\left(e^{i \pi} r\right)-\Pi\left(e^{-i \pi} r\right)=0$ which results in the vanishing of the first part in Eq. (135). We are therefore left with the two semicircle integrals which we can combine in a different manner

$$
\rho(s)=\frac{-2 \pi i}{\sqrt{s}} \int_{C_{-}+C_{+}} I_{1}(z \sqrt{s}) \Pi(z) z^{2} d z-\frac{2}{\sqrt{s}} \int_{C_{-} C_{+}} K_{1}(z \sqrt{s}) \Pi(z) z^{2} d z .
$$


In expanding the first integrand for small values of $r=|z|$ we obtain

$$
I_{1}(z \sqrt{s}) \Pi(z) z^{2}=\frac{\sqrt{s}}{2(2 \pi)^{4} z}+O(z),
$$

the integral results in

$$
\int_{C_{-}+C_{+}} I_{1}(z \sqrt{s}) \Pi(z) z^{2} d z=\frac{\sqrt{s}}{2(2 \pi)^{4}}[\ln r+i \varphi]_{-\pi}^{\pi}=\frac{i \sqrt{s}}{16 \pi^{3}} .
$$

For the second integrand the expansion results in many more terms,

$$
\begin{aligned}
K_{1}(z \sqrt{s}) \Pi(z) z^{2}= & \frac{1}{(2 \pi)^{4} z^{3} \sqrt{s}}+\frac{1}{4(2 \pi)^{4} z \sqrt{s}}\left(m^{2} \ln \left(m^{2}\right)+s \ln s\right) \\
& +\frac{m^{2}+s}{2(2 \pi)^{4} z \sqrt{s}}\left(\ln \left(\frac{z}{2}\right)+\gamma_{E}-\frac{1}{2}\right) .
\end{aligned}
$$

However one has

$$
\begin{aligned}
\int_{C_{-}-C_{+}} \frac{d z}{z^{3}} & =\left[\frac{-1}{2 z^{2}}\right]_{C_{-}-C_{+}}=\left[\frac{-1}{2 r^{2} e^{2 i \varphi}}\right]_{\varphi=-\pi}^{0}-\left[\frac{-1}{2 r^{2} e^{2 i \varphi}}\right]_{\varphi=0}^{\pi}=0 \\
\int_{C_{-}-C_{+}} \frac{d z}{z} & =[\ln z]_{C_{-} C_{+}}=[\ln \epsilon+i \varphi]_{\varphi=-\pi}^{0}-[\ln \epsilon+i \varphi]_{\varphi=0}^{\pi}=\pi-\pi=0 .
\end{aligned}
$$

The only non-vanishing contribution is given by

$$
\int_{C_{-}-C_{+}} \frac{d z}{z} \ln z=\frac{1}{2}\left[\ln ^{2} z\right]_{C_{-}-C_{+}}=\pi^{2} .
$$

Therefore, we obtain

$$
\int_{C_{-}-C_{+}} K_{1}(z \sqrt{s}) \Pi(z) z^{2} d z=\frac{m^{2}+s}{32 \pi^{2} \sqrt{s}} .
$$

The spectral density, finally, reads again

$$
\rho(s)=\frac{-2 \pi i}{\sqrt{s}} \frac{i \sqrt{s}}{16 \pi^{3}}-\frac{2}{\sqrt{s}} \frac{m^{2}+s}{32 \pi^{2} \sqrt{s}}=\frac{1}{16 \pi^{2}}\left(1-\frac{m^{2}}{s}\right)
$$

as in Eq. (132).

\subsection{Large $s$ expansion for massive one- and multiloop diagrams}

A second example that we treat is the one-loop diagram with two different masses $m_{1}$ and $m_{2}$. We start again with the calculation in momentum space to obtain

$$
\tilde{\Pi}(p)=\frac{\Gamma(2-D / 2)}{(4 \pi)^{D / 2}} \int_{0}^{1}\left(x(1-x) p^{2}+(1-x) m_{1}^{2}+x m_{2}^{2}\right)^{D / 2-2} d x .
$$


Again the discontinuity can be calculated already on the level of integrands. Using Eq. (C15) we obtain

$$
\begin{aligned}
& \operatorname{Disc}\left(-x(1-x) s+(1-x) m_{1}^{2}+x m_{2}^{2}\right)^{-\varepsilon}= \\
& =\frac{2 \pi i}{\Gamma(\varepsilon) \Gamma(1-\varepsilon)}\left(x(1-x) s-(1-x) m_{1}^{2}-x m_{2}^{2}\right)^{-\varepsilon} \theta\left(x(1-x) s-(1-x) m_{1}^{2}-x m_{2}^{2}\right) .
\end{aligned}
$$

In order to write down the spectral density we have to determine the limits for $x$ given by the positiveness of the theta function. The zeros of the argument of the theta function can be calculated to be

$$
x_{1,2}=\frac{1}{2}\left(1+\frac{m_{1}^{2}}{s}-\frac{m_{2}^{2}}{s}\right) \pm \frac{1}{2} \sqrt{\lambda\left(1, m_{1}^{2} / s, m_{2}^{2} / s\right)}
$$

where

$$
\lambda(x, y, z)=x^{2}+y^{2}+z^{2}-2 x y-2 x z-2 y z
$$

is Källén's lambda function. In terms of these two zeros $x_{1} \leq x_{2}$ we can write the condition for the argument of the theta function as

$$
\left(x-x_{1}\right)\left(x_{2}-x\right) s \geq 0 \quad \Rightarrow \quad x_{1} \leq x \leq x_{2}
$$

The two zeros take real values if

$$
s^{2} \lambda\left(1, m_{1}^{2} / s, m_{2}^{2} / s\right)=\left(s-\left(m_{1}-m_{2}\right)^{2}\right)\left(s-\left(m_{1}+m_{2}\right)^{2}\right) \geq 0
$$

This is the case for $s \geq\left(m_{1}+m_{2}\right)^{2}$ or $s \leq\left(m_{1}-m_{2}\right)^{2}$. We do not consider the part of the spectral density up to the pseudothreshold $\left(m_{1}-m_{2}\right)^{2}$ because in this case both $x_{1}$ and $x_{2}$ are negative. Starting from the threshold $\left(m_{1}+m_{2}\right)^{2}$, both $x_{1}$ and $x_{2}$ take values between 0 and 1. We obtain

$$
\begin{aligned}
\rho(s) & =\frac{1}{2 \pi i} \operatorname{Disc} \tilde{\Pi}(-s)=\frac{1}{(4 \pi)^{2-\varepsilon} \Gamma(1-\varepsilon)} \int_{x_{1}}^{x_{2}} s^{-\varepsilon}\left(x-x_{1}\right)^{-\varepsilon}\left(x_{2}-x\right)^{-\varepsilon} d x \\
& =\frac{s^{-\varepsilon} \Gamma(1-\varepsilon)}{(4 \pi)^{2-\varepsilon} \Gamma(2-2 \varepsilon)} \sqrt{\lambda\left(1, m_{1}^{2} / s, m_{2}^{2} / s\right)}
\end{aligned}
$$

where we have used the substitution $x^{\prime}=\left(x-x_{1}\right) /\left(x_{2}-x_{1}\right)$ and Euler's Beta function

$$
B(\alpha, \beta)=\frac{\Gamma(\alpha) \Gamma(\beta)}{\Gamma(\alpha+\beta)}=\int_{0}^{1} x^{\alpha-1}(1-x)^{\beta-1} d x
$$

For $D=4$, i.e. $\varepsilon=0$, we obtain

$$
\rho(s)=\frac{1}{(4 \pi)^{2}} \sqrt{1-2\left(\frac{m_{1}^{2}}{s}+\frac{m_{2}^{2}}{s}\right)+\left(\frac{m_{1}^{2}}{s}-\frac{m_{2}^{2}}{s}\right)^{2}} .
$$


For the case $m_{2}=0$ we recover the spectral density of the previous example. If we consider an expansion in terms of large values of $s$ compared to the threshold value $s=\left(m_{1}+m_{2}\right)^{2}$, i.e. if we expand in small values of $z$ for $z=\left(m_{1}+m_{2}\right)^{2} / s$, we obtain

$$
\rho(s)=\frac{1}{(4 \pi)^{2}}\left(1-\frac{m_{1}^{2}+m_{2}^{2}}{s}+O\left(\frac{1}{s^{2}}\right)\right) .
$$

The same result shall now be calculated in a different way. Consider the correlator function

$$
\tilde{\Pi}(p)=2 \pi^{\lambda+1} \int_{0}^{\infty}\left(\frac{p x}{2}\right)^{-\lambda} J_{\lambda}(p x) D\left(x, m_{1}\right) D\left(x, m_{2}\right) x^{2 \lambda+1} d x .
$$

We can expand the propagators $D\left(x, m_{i}\right)$ in $m_{i} / \sqrt{s}$, leading to pure powers in $x$. Starting from Eq. (86)), the integration of the Bessel function of the first kind with (non-integer) powers of $x$ can be cast into a more appropriate form

$$
\int_{0}^{\infty} x^{\mu}\left(\frac{p x}{2}\right)^{-\lambda} J_{\lambda}(p x) x^{2 \lambda+1} d x=\left(\frac{p}{2}\right)^{-2 \lambda-2-\mu} \frac{\Gamma(\lambda+1+\mu / 2)}{2 \Gamma(-\mu / 2)} .
$$

We can then immediately calculate the discontinuity and obtain

$$
\frac{1}{2 \pi i} \operatorname{Disc} \int_{0}^{\infty} x^{\mu}\left(\frac{p x}{2}\right)^{-\lambda} J_{\lambda}(p x) x^{2 \lambda+1} d x=\frac{(s / 4)^{-\lambda-1-\mu / 2}}{2 \Gamma(-\lambda-\mu / 2) \Gamma(-\mu / 2)} .
$$

Using the expansion of the Bessel functions $K_{\lambda}(z)$ at small values of $z$, the expansion of the propagator for $\lambda$ close to $\lambda=1$ for small mass $m$ (compared to $\sqrt{s}$ ) reads

$$
\begin{aligned}
& D(x, m)=\frac{(m x)^{\lambda} K_{\lambda}(m x)}{(2 \pi)^{\lambda+1} x^{2 \lambda}} \\
& \quad=\frac{2^{\lambda-1} \Gamma(\lambda)}{(2 \pi)^{\lambda+1} x^{2 \lambda}}\left(1-\frac{1}{1-\lambda}\left(\frac{m x}{2}\right)^{2}-\frac{\Gamma(1-\lambda)}{\Gamma(1+\lambda)}\left(\frac{m x}{2}\right)^{2 \lambda}+O\left((m x)^{4},(m x)^{2+2 \lambda}\right)\right) .
\end{aligned}
$$

Eq. (155) contains two of these propagators. We now expand up to the power $m^{2}$ and keep $\lambda$ close to 1 . We obtain a zeroth order contribution, two contributions of the order $m^{2}$, and two contributions of the order $m^{2(1+\lambda)}$. We consider these contributions in turn.

- zeroth order $(\mu=-4 \lambda)$ :

$$
\frac{2 \pi^{\lambda+1}(s / 4)^{\lambda-1}}{2 \Gamma(2 \lambda) \Gamma(\lambda)}\left(\frac{2^{\lambda-1} \Gamma(\lambda)}{(2 \pi)^{\lambda+1}}\right)^{2} \rightarrow \frac{2 \pi^{2}}{2 \Gamma(2) \Gamma(1)}\left(\frac{\Gamma(1)}{(2 \pi)^{2}}\right)^{2}=\frac{1}{16 \pi^{2}}
$$

- second order $(\mu=2-4 \lambda, i=1,2)$ :

$$
\begin{aligned}
& \frac{2 \pi^{\lambda+1}(s / 4)^{\lambda-2}}{2 \Gamma(2 \lambda-1) \Gamma(\lambda-1)}\left(\frac{2^{\lambda-1} \Gamma(\lambda)}{(2 \pi)^{\lambda+1}}\right)^{2} \frac{\left(m_{i}^{2} / 4\right)}{1-\lambda} \quad(\Gamma(\lambda)=(\lambda-1) \Gamma(\lambda-1)) \\
& =\frac{-2 \pi^{\lambda+1}(s / 4)^{\lambda-2}}{2 \Gamma(2 \lambda-1) \Gamma(\lambda)}\left(\frac{2^{\lambda-1} \Gamma(\lambda)}{(2 \pi)^{\lambda+1}}\right)^{2} \frac{m_{i}^{2}}{4} \rightarrow \frac{-2 \pi^{2}(s / 4)^{-1}}{2 \Gamma(1) \Gamma(1)}\left(\frac{\Gamma(1)}{(2 \pi)^{\lambda+1}}\right)^{2} \frac{m_{i}^{2}}{4}=\frac{-m_{i}^{2}}{16 \pi^{2} s}
\end{aligned}
$$


- order $m^{2(1+\lambda)}$ (also called "second order primed", $\mu=-2 \lambda, i=1,2$ ):

$$
-\frac{(s / 4)^{-1}}{\Gamma(-\lambda-\mu / 2) \Gamma(-\mu / 2)}\left(\frac{2^{\lambda-1} \Gamma(\lambda)}{(2 \pi)^{\lambda+1}}\right)^{2} \frac{2 \Gamma(1-\lambda)}{\Gamma(1+\lambda)}\left(\frac{m_{i}^{2}}{4}\right)^{\lambda} .
$$

Since the third contribution vanishes for $\mu \rightarrow-2 \lambda$, only the first two contributions have to be taken into account. One ends up with

$$
\rho(s)=\frac{1}{16 \pi^{2}}\left(1-\frac{m_{1}^{2}}{s}-\frac{m_{2}^{2}}{s}+O\left(\frac{1}{s^{2}}\right)\right)
$$

in agreement with the result obtained earlier. Moreover, we can predict the result for a $n$-loop sunrise-type diagram with different masses $m_{1}, \ldots m_{n+1}$ in four space-time dimensions where $\mu=-2(n+1) \lambda$ (zeroth order contribution), $\mu=2-2(n+1) \lambda$ (second order contribution), and $\mu=-2 n \lambda$ (second order primed contribution which does not vanish for $n>1$ ),

$$
\begin{aligned}
\rho(s)= & 2 \pi^{\lambda+1}\left(\frac{2^{\lambda-1} \Gamma(\lambda)}{(2 \pi)^{\lambda+1}}\right)^{n+1} \frac{(s / 4)^{n \lambda-1}}{2 \Gamma((n+1) \lambda) \Gamma(n \lambda)}(1+ \\
& \left.+\frac{((n+1) \lambda-1)(n \lambda-1)}{1-\lambda} \sum_{i=1}^{n+1} \frac{m_{i}^{2}}{s}-\frac{\Gamma((n+1) \lambda) \Gamma(1-\lambda)}{\Gamma((n-1) \lambda) \Gamma(1+\lambda)} \sum_{i=1}^{n+1}\left(\frac{m_{i}^{2}}{s}\right)^{\lambda}+O\left(\frac{1}{s^{2}}\right)\right) .
\end{aligned}
$$

The second and third term have to be expanded in $\varepsilon=1-\lambda$ in order that the singularity in $\varepsilon$ cancels. After a few simplifications using expecially

$$
\psi(n+1)=\psi(n)+\frac{1}{n}
$$

for the polygamma function, we finally obtain for $\lambda=1$

$$
\rho(s)=\frac{s^{n-1}}{(4 \pi)^{2 n} n !(n-1) !}\left(1+n \sum_{i=1}^{n+1}\left((n-1)\left(\ln \left(\frac{m_{i}^{2}}{s}\right)+2\left(\psi(n)+\gamma_{E}\right)\right)-n\right) \frac{m_{i}^{2}}{s}+O\left(\frac{1}{s^{2}}\right)\right) .
$$

This result is also valid for $n=1$ and again confirms our previous results. Note that $\left(\psi(n)+\gamma_{E}\right)$ is a rational number because Euler's constant cancels out. Indeed, we have

$$
\psi(n)+\gamma_{E}=\sum_{k=1}^{n-1} \frac{1}{k} .
$$

Note, however, that for $n>1$ logarithmic contributions $\ln \left(m_{i}^{2} / s\right)$ appear in the spectral density. 


\section{Non-standard propagators and other exotic settings}

In this section we deal with modifications of the standard propagators in sunrise-type diagrams in any space-time dimension. First we consider odd-dimensional space-time which in most of the cases allows one to calculate the sunrise-type integrals in closed form. Returning to spacetime dimensions close to four, we then deal with larger powers of propagators leading to larger values for the Bessel function indices. In this case one can apply recurrence relations to reduce integrals containing higher powers of the propagators to a set of master integrals 39]. The master integrals themselves show Laplace-type asymptotics (for a definition see e.g. Ref. [171]). Finally we deal with nontrivial numerators in two examples involving vacuum bubbles.

\subsection{Odd-dimensional case}

Compared to four-dimensional space-time, integrals containing products of Bessel functions in odd dimensional space-time are analytically solvable because the Bessel functions simplify significantly. It is interesting to note that the evaluation of Eq. (18) can be done in a closed form for any number of internal lines in odd-dimensional space-time. One might suspect that this special case is too simple to have any practical applications. However, at the end of this subsection we will refer to many applications from various fields of physics involving odddimensional space-time. As the simplest example we take three-dimensional space-time $D \rightarrow$ $D_{0}=3$. For $D_{0}=3$ (and $\left.\lambda_{0}=\left(D_{0}-2\right) / 2=1 / 2\right)$ the propagator in Eq. (77) reads

$$
D(x, m) \rightarrow D_{3}(x, m)=\frac{\sqrt{m x} K_{1 / 2}(m x)}{(2 \pi)^{3 / 2} x}=\frac{e^{-m x}}{4 \pi x} .
$$

With $\lambda=\lambda_{0}=1 / 2$ the weight function becomes

$$
\left(\frac{p x}{2}\right)^{-1 / 2} J_{1 / 2}(p x)=\frac{2}{\sqrt{\pi}} \frac{\sin (p x)}{p x}
$$

after angular integration. The explicit result for the $(n+1)$-line sunrise diagram is then given by the integral

$$
\begin{aligned}
\tilde{\Pi}(p) & =4 \pi \int_{0}^{\infty} \frac{\sin (p x)}{p x} \frac{e^{-M x}}{(4 \pi x)^{n-1}}(\mu x)^{2 \epsilon} d x \\
& =\frac{\Gamma(1-n+2 \epsilon)}{2 i p(4 \pi)^{n}}\left[(M-i p)^{n-1-2 \epsilon}-(M+i p)^{n-1-2 \epsilon}\right] \mu^{2 \epsilon}
\end{aligned}
$$

where $\epsilon$ is used for regularization and $M=\sum m_{i}$. Note that we have used an unorthodox regularization method by multiplying a factor $(\mu x)^{2 \varepsilon}$. We will return to this point later on. 
We consider some particular cases of Eq. (169) for different values of $n$. For $n=0$ we simply recover the (Euclidean) propagator function $\tilde{\Pi}(p)=\left(M^{2}+p^{2}\right)^{-1}$ with the discontinuity

$$
\rho(s)=\frac{\operatorname{Disc} \tilde{\Pi}(p)}{2 \pi i}=\frac{1}{2 \pi i}\left(\left.\tilde{\Pi}(p)\right|_{p^{2}=s \exp (-i \pi)}-\left.\tilde{\Pi}(p)\right|_{p^{2}=s \exp (i \pi)}\right)=\delta\left(s-m^{2}\right)
$$

where $s$ is the squared energy, $s=-p^{2}$. As remarked on earlier it is appropriate to call this expression the spectral density associated with the diagram. For $n=1$ the answer for the polarization function $\tilde{\Pi}(p)$ is still finite (no regularization is required) and is given by

$$
\tilde{\Pi}(p)=\frac{1}{8 \pi i p} \ln \left(\frac{M+i p}{M-i p}\right) \text {. }
$$

The spectral density, i.e. the discontinuity of Eq. (171) across the cut in the complex $p^{2}$-plane is given by

$$
\rho(s)=\frac{1}{8 \pi \sqrt{s}} \theta\left(s-M^{2}\right), \quad s=-p^{2}, \quad s>0
$$

which is nothing but the three-dimensional two-particle phase space. This can be immediately checked by direct computation. The cases with $n>1$ have more structure and therefore are more interesting. For the genuine sunrise diagram with $n=2$, Eq. (169) leads to

$$
\tilde{\Pi}(p)=\frac{1}{32 \pi^{2}}\left(\frac{1}{\epsilon}-\frac{M}{i p} \ln \left(\frac{M+i p}{M-i p}\right)-\ln \left(\frac{M^{2}+p^{2}}{\mu^{2}}\right)\right) .
$$

While in Eq. (173) the arbitrary scale $\mu^{2}$ appears due to regularization, the spectral density

$$
\rho(s)=\frac{\sqrt{s}-M}{32 \pi^{2} \sqrt{s}} \theta\left(s-M^{2}\right)
$$

is independent of this scale. This is because the spectral density is again finite and, therefore, independent of the regularization used. The general formula for the spectral density for any $n>0$ in $D=3$ can be extracted from Eq. (169). It reads

$$
\rho(s)=\frac{(\sqrt{s}-M)^{n-1}}{2(4 \pi)^{n}(n-1) ! \sqrt{s}} \theta\left(s-M^{2}\right) .
$$

We now want to comment on the relation between the momentum subtraction and our unorthodox dimensional regularization. Taking Eq. (169) for $n=2$ with momentum subtraction at the origin, one obtains

$$
\tilde{\Pi}(p)=\int_{0}^{\infty}\left(\frac{\sin (p x)}{p x}-1\right) \frac{e^{-M x}}{(4 \pi)^{2} x}(\mu x)^{2 \epsilon} d x
$$

which is UV-finite even for $\epsilon=0$ because there is no singularity at the origin. For practical computations it is convenient to keep the factor $(\mu x)^{2 \epsilon}$ in the integrand since this factor gives 
a meaning to each of the two terms in the round brackets in Eq. (176). Using this factor we obtain

$$
\begin{aligned}
\tilde{\Pi}(p) & =\int_{0}^{\infty}\left(\frac{\sin (p x)}{p x}-1\right) \frac{e^{-M x}}{(4 \pi)^{2} x}\left(\mu^{2} x^{2}\right)^{\epsilon} d x \\
& =\frac{\Gamma(-1+2 \epsilon)}{2 i p(4 \pi)^{2}}\left[(M-i p)^{1-2 \epsilon}-(M+i p)^{1-2 \epsilon}\right] \mu^{2 \epsilon}-\frac{\Gamma(2 \epsilon)}{(4 \pi)^{2}}\left(\frac{\mu}{M}\right)^{2 \epsilon} \\
& =-\frac{1}{32 \pi^{2}}\left\{\frac{M}{i p} \ln \left(\frac{M+i p}{M-i p}\right)+\ln \left(\frac{M^{2}+p^{2}}{M^{2}}\right)\right\} .
\end{aligned}
$$

The poles cancel in this expression and the arbitrary scale $\mu$ changes to $M$. This corresponds to a transition from MS-type renormalization schemes to the momentum subtraction scheme (in this particular case with a subtraction at the origin). Since the spectral density $\rho(s)$ is finite it can be computed using any regularization scheme as can be seen from Eqs. (173) and (177).

We mention that in the three-dimensional case the spectral density $\rho(s)$ can also be found for general values of $n$ by traditional methods since the three-dimensional case is sufficiently simple. By using the convolution for the evaluation of spectral densities one stays in the same class of functions, i.e. polynomials in the variable $\sqrt{s}$ divided by $\sqrt{s}$. The general form of the convolution equation in $D$-dimensional space-time reads

$$
\Phi_{n}(s)=\int \Phi_{k}\left(s_{1}\right) \Phi_{p}\left(s_{2}\right) \Phi_{1}\left(s, s_{1}, s_{2}\right) d s_{1} d s_{2}, \quad k+p+1=n .
$$

For the particular case of three-dimensional space-time the kernel $\Phi_{1}\left(p^{2}, m_{1}^{2}, m_{2}^{2}\right)$ is given by

$$
(2 \pi)^{2} \Phi_{1}\left(p^{2}, m_{1}^{2}, m_{2}^{2}\right)=\int \delta\left(k^{2}-m_{1}^{2}\right) \delta\left((p-k)^{2}-m_{2}^{2}\right) d^{3} k
$$

or explicitly by

$$
\Phi_{1}\left(s, s_{1}, s_{2}\right)=\frac{1}{8 \pi \sqrt{s}} \theta\left(s-\left(\sqrt{s_{1}}+\sqrt{s_{2}}\right)^{2}\right) .
$$

Eq. (180) can be seen to be the two-particle phase space in three dimensions (cf. Eq. (172) ). This is a rather simple example. However, our technique retains its efficiency for large $n$.

We list some potential applications of the general results obtained in this paragraph for odddimensional space-time. In three space-time dimensions our results can be used to compute phase space integrals for particles in jets where the momentum along the direction of the jet is fixed [104]. Another application can be found in phase transitions, for instance the threedimensional QCD which emerges as the high temperature limit of the ordinary theory of strong interactions for the quark-gluon plasma (see e.g. 34, 93, 172, 92]). Three-dimensional models are also used to study the question of dynamical mass generation and the infrared structure of the models of Quantum Field Theory in general [87, 173, 174] and some problems of QCD [175]. 
Note that particular models of different space-time dimensions are very useful because their properties may be simpler and may thus allow one to study general features of the underlying field theory. For example, in six-dimensional space-time the simplest model of quantum field theory $\phi^{3}$ is asymptotically free and can be used for simulations of some features of QCD [176].

As we have already mentioned before, closed form analytical results can be obtained for odd dimensional space-time $D=3,5,7, \ldots$ For example, the propagator in five-dimensional space-time $(\lambda=3 / 2)$ reads

$$
D(x, m) \rightarrow D_{5}(x, m)=\frac{(m x)^{3 / 2} K_{3 / 2}(m x)}{(2 \pi)^{5 / 2} x^{3}}=\frac{e^{-m x}}{8 \pi^{2} x^{3}}(1+m x)
$$

which assures that the basic integration can be performed in terms of elementary functions (powers and logarithms) again.

Applications for odd space-time dimensions other than three can be found in some models in unified field theories, or also in a general analysis of the divergence structure in QFT. Fivedimensional models of QFT are rather popular for general purposes [177, 178, 179, 180]. They have useful applications for Yang-Mills theories in five-dimensional space-time where the UV structure of the models can be analyzed [181].

Finally, non-standard space-time dimensions are useful in order to obtain estimates for the standard case $D_{0}=4$. The feature that $K_{\nu}(x)>K_{\mu}(x)$ holds for modified Bessel functions of the second kind when $\nu>\mu$ and for positive $x$-arguments leads to $K_{3 / 2}(x)>K_{1}(x)>$ $K_{1 / 2}(x)$ which can be used for stringent numerical estimates for integrals containing these Bessel functions.

We conclude this paragraph by noting that the $x$-space techniques allow one to compute sunrise-type diagrams in closed form in terms of elementary functions as long as one is dealing with odd-dimensional space-times. The resulting expressions are rather simple and can be directly used for applications. Having the complete formulas at hand, there is no need to expand in the parameters of the diagram such as masses or external momenta.

\subsection{Large powers of propagators}

In many physics applications one encounters large powers of propagators. Recent examples are the calculation of the corrections to $B^{0}-\bar{B}^{0}$ mixing in perturbative QCD [182, 183] (see Fig. 121) or the large mass expansion for the contribution of charged scalars to the muon anomalous magnetic moment [184]. When calculating moments of the spectral density of such a diagram by using packages like MATAD for the automatic calculation of Feynman diagrams [185, 186, 187], 


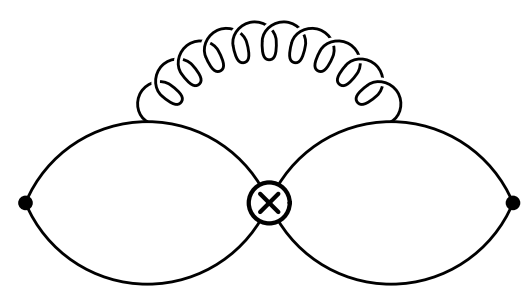

Figure 12: A non-factorizable diagram at NLO for $B^{0}-\bar{B}^{0}$ mixing

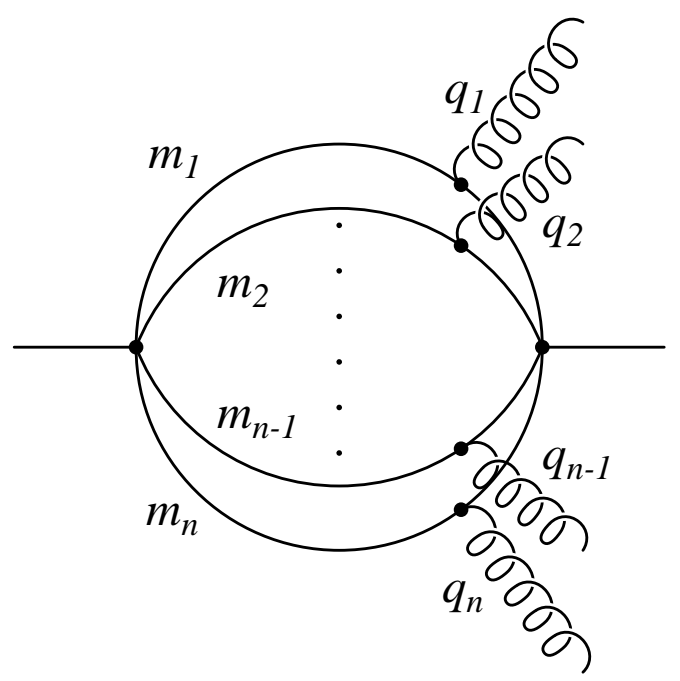

Figure 13: Sunrise-type diagram with additional external momenta

high powers of propagators are generated. Another example are sunrise-type diagrams with external lines at small momenta $q_{i}$ (see Fig. 13). These diagrams appear in calculations when there are weak external fields as, for example, in sum rule applications [188, 189] or in special cases of high precision calculations in ChPT [107].

In momentum space larger powers of propagators are treated through recurrence relations based on the integration-by-parts technique 190, 191, 192. In configuration space larger powers of the propagators give rise to larger values of the indices for the corresponding Bessel functions. We now describe a technique using recurrence relations which allows one to reduce large indices of Bessel functions [39].

Note that the direct reduction of a sunrise-type diagram to a standard set of master integrals with the help of algebraic computer systems is rather time-consuming with present momentum space techniques. In practice the computation proceeds through the use of a table of integrals with given powers of the denominators. One would have to set up a three-dimensional table for a given total power $N$. The number of entries (even when accounting for the appropriate 
symmetries) still grows as fast as $N^{3}$ which is large for the large values of $N$ needed in some present applications. Within our method one first re-expresses the relevant integrals through a one-parameter set of integrals which are then solved explicitly. For large $N$ the number of entries increases as a first power of $N$ (the number of elements for the $I_{0}(q)$ basis is given by $2[N / 2]-5$ where $[z]$ is an integer part of $z$ ) which considerably reduces the time consumption in a computer evaluation.

In order to show the applicability of our method we shall calculate three examples that were solved before with the help of momentum space techniques [136]. In Ref. [136] Broadhurst considered general three-loop bubbles $B_{N}$. A subclass of these are the sunrise-type three-loop bubbles. In momentum space they read

$$
\begin{aligned}
& B_{N}\left(0,0, n_{3}, n_{4}, n_{5}, n_{6}\right)=\int \frac{d^{D} k d^{D} l d^{D} p}{m^{3 D}\left(\pi^{D / 2} \Gamma(3-D / 2)\right)^{3}} \times \\
& \quad \times \frac{m^{2 n_{3}}}{\left((p+k)^{2}+m^{2}\right)^{n_{3}}} \frac{m^{2 n_{4}}}{\left((p+l)^{2}+m^{2}\right)^{n_{4}}} \frac{m^{2 n_{5}}}{\left((p+k+l)^{2}+m^{2}\right)^{n_{5}}} \frac{m^{2 n_{6}}}{\left(p^{2}+m^{2}\right)^{n_{6}}}
\end{aligned}
$$

with two propagators absent $\left(n_{1}=n_{2}=0\right)$. Actually we choose the remaining indices $n_{3}$, $n_{4}, n_{5}$, and $n_{6}$ such that the results become finite. We shall not only calculate the finite part but also the part porportional to $\varepsilon$ in order to be able to compare with [136. Written in configuration space, the particular subset of bubble diagrams $B_{N}$ is given by

$$
\begin{aligned}
& B_{N}\left(0,0, n_{3}, n_{4}, n_{5}, n_{6}\right)=\frac{2\left(64 \pi^{4}\right)^{2-\varepsilon}}{(\Gamma(1+\varepsilon))^{3} \Gamma(2-\varepsilon)} m^{2\left(n_{3}+n_{4}+n_{5}+n_{6}\right)-12+6 \varepsilon} \times \\
& \quad \times \int_{0}^{\infty} D^{\left(n_{3}-1\right)}(x, m) D^{\left(n_{4}-1\right)}(x, m) D^{\left(n_{5}-1\right)}(x, m) D^{\left(n_{6}-1\right)}(x, m) x^{2 \lambda+1} d x .
\end{aligned}
$$

It is clear that one will end up with integrals of products of four Bessel functions with noninteger indices and a non-integer power of $x$. We first discuss three examples in the next three subsections which will be followed by more general considerations on a general reduction procedure which allows one to reduce all integrals containing products of Bessel functions to the following set of two master integrals,

$$
L_{4}(r):=\int_{0}^{\infty}\left(K_{0}(\xi)\right)^{4} \xi^{r} d \xi \quad \text { and } \quad L_{4}^{l}(r):=\int_{0}^{\infty}\left(K_{0}(x)\right)^{4} \xi^{r} \ln \left(e^{\gamma_{E}} \xi / 2\right) d x
$$

where the index "l" in $L_{4}^{l}(r)$ reflects the logarithm appearing in the integrand. We will then add some considerations on the basic integrals $L_{4}(r)$ and $L_{4}^{l}(r)$.

\subsection{The example $B_{N}(0,0,2,2,2,2)$}

We start with the example which is represented by the diagram in Fig. 14(b). Each of the lines is modified (indicated by the dots on the lines) which means that instead of the propagators 

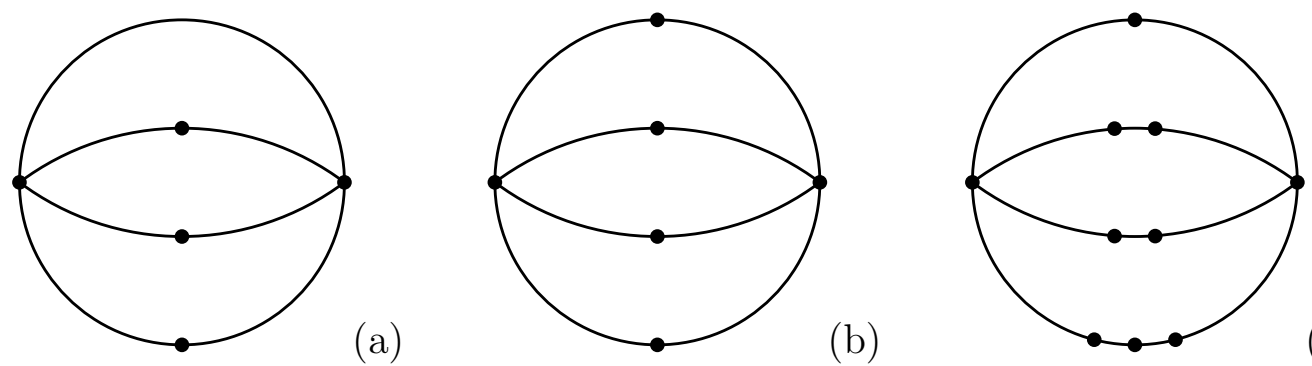

(c)

Figure 14: The diagrams for $B_{N}(0,0,2,2,2,1), B_{N}(0,0,2,2,2,2)$, and $B_{N}(0,0,2,3,3,4)$.

$D(x, m)$ we have to use

$$
D^{(1)}(x, m)=\int \frac{d^{D} p}{(2 \pi)^{D}} \frac{e^{i p_{\mu} x^{\mu}}}{\left(p^{2}+m^{2}\right)^{2}}=\frac{(x / m)^{1-\lambda}}{2(2 \pi)^{\lambda+1}} K_{\lambda-1}(m x)=\frac{(x / m)^{\varepsilon}}{2(2 \pi)^{2-\varepsilon}} K_{-\varepsilon}(m x) .
$$

One obtains $(\xi=m x)$

$$
B_{N}(0,0,2,2,2,2)=\frac{2^{1-2 \varepsilon}}{(\Gamma(1+\varepsilon))^{3} \Gamma(2-\varepsilon)} \int_{0}^{\infty}\left(K_{-\varepsilon}(\xi)\right)^{4} \xi^{3+2 \varepsilon} d \xi .
$$

We now can use the general formula [135]

$$
\left[\frac{\partial K_{\nu}(z)}{\partial \nu}\right]_{\nu= \pm n}= \pm \frac{1}{2} n ! \sum_{k=0}^{n-1}\left(\frac{z}{2}\right)^{k-n} \frac{K_{k}(z)}{k !(n-k)}, \quad n \in\{0,1, \ldots\}
$$

to expand the Bessel function in a series with respect to its index. In case of $K_{-\varepsilon}(z)$, however, the first derivative vanishes and we obtain $K_{-\varepsilon}(z)=K_{0}(z)+O\left(\varepsilon^{2}\right)$. Therefore, in expanding

$$
\begin{aligned}
\frac{2^{1-2 \varepsilon}\left(K_{-\varepsilon}(\xi)\right)^{4} \xi^{3+2 \varepsilon}}{(\Gamma(1+\varepsilon))^{3} \Gamma(2-\varepsilon)} & =2\left(K_{0}(\xi)\right)^{4} \xi^{3}\left(1+\left(1+2 \gamma_{E}-2 \ln 2+2 \ln \xi\right) \varepsilon+O\left(\varepsilon^{2}\right)\right)= \\
& =2\left(K_{0}(\xi)\right)^{4} \xi^{3}\left(1+\left(1+2 \ln \left(e^{\gamma_{E}} \xi / 2\right)\right) \varepsilon+O\left(\varepsilon^{2}\right)\right)
\end{aligned}
$$

we obtain

$$
\begin{aligned}
B_{N}(0,0,2,2,2,2) & =2(1+\varepsilon) \int_{0}^{\infty}\left(K_{0}(\xi)\right)^{4} \xi^{3} d \xi+4 \varepsilon \int_{0}^{\infty}\left(K_{0}(\xi)\right)^{4} \xi^{3} \ln \left(e^{\gamma_{E}} \xi / 2\right) d \xi= \\
& =2(1+\varepsilon) L_{4}(3)+4 \varepsilon L_{4}^{l}(3) .
\end{aligned}
$$

We have compared our numerical result with the analytical result in [136 and have found agreement. In fact, from the transcendality structure of the results in [136] one can numerically obtain the appropriate coefficients multiplying the transcendentals. One finds [39]

$$
\begin{aligned}
& L_{4}(3)=-\frac{3}{16}+\frac{7}{32} \zeta(3), \\
& L_{4}^{l}(3)=\frac{3}{32}+\frac{3}{4} \operatorname{Li}_{4}\left(\frac{1}{2}\right)-\frac{17 \pi^{4}}{1920}-\frac{\pi^{2}}{32}(\ln 2)^{2}+\frac{1}{32}(\ln 2)^{4}+\frac{49}{128} \zeta(3) .
\end{aligned}
$$




\subsection{The example $B_{N}(0,0,2,2,2,1)$}

In the diagram of Fig. 14(a) one of the lines is not modified. Therefore, we have to deal with one regular propagator factor

$$
D^{(0)}(x, m)=D(x, m)=\frac{(x / m)^{-\lambda}}{(2 \pi)^{\lambda+1}} K_{\lambda}(m x)=\frac{(x / m)^{\varepsilon-1}}{(2 \pi)^{2-\varepsilon}} K_{1-\varepsilon}(m x) .
$$

In this case we obtain

$$
B_{N}(0,0,2,2,2,1)=\frac{2^{2-2 \varepsilon}}{(\Gamma(1+\varepsilon))^{3} \Gamma(2-\varepsilon)} \int_{0}^{\infty}\left(K_{-\varepsilon}(\xi)\right)^{3} K_{1-\varepsilon}(\xi) \xi^{2+2 \varepsilon} d \xi .
$$

Using Eq. (187) one has

and

$$
K_{1-\varepsilon}(\xi)=K_{1}(\xi)-\frac{\varepsilon}{\xi} K_{0}(\xi)+O\left(\varepsilon^{2}\right)
$$

$$
\frac{2^{2-2 \varepsilon}\left(K_{-\varepsilon}(\xi)\right)^{3} K_{1-\varepsilon}(\xi) \xi^{2+2 \varepsilon}}{(\Gamma(1+\varepsilon))^{3} \Gamma(2-\varepsilon)}=4 \xi^{2}\left(1+\left(1-\frac{1}{\xi}+\ln \left(e^{\gamma_{E}} \xi / 2\right)\right) \varepsilon+O\left(\varepsilon^{2}\right)\right) .
$$

The result reads

$$
\begin{aligned}
B_{N}(0,0,2,2,2,1)= & 4(1+\varepsilon) \int_{0}^{\infty}\left(K_{0}(\xi)\right)^{3} K_{1}(\xi) \xi^{2} d \xi-4 \varepsilon \int_{0}^{\infty}\left(K_{0}(\xi)\right)^{4} \xi d \xi \\
& +8 \varepsilon \int_{0}^{\infty}\left(K_{0}(\xi)\right)^{3} K_{1}(\xi) \xi^{2} \ln \left(e^{\gamma_{E}} \xi / 2\right) d \xi
\end{aligned}
$$

This result is not yet written in terms of $L_{4}(r)$ and $L_{4}^{l}(r)$ and will be dealt with after introduction of the reduction procedure (The result for $\varepsilon=0$ was given earlier in Sec. (3.6)).

\subsection{The example $B_{N}(0,0,2,3,3,4)$}

In order to demonstrate the power of the configuration space technique also in a more complex setting we finally choose the diagram in Fig. 14(c) as an example. The modified propagators now read

$$
D^{(2)}(x, m)=\frac{(x / m)^{1+\varepsilon}}{8(2 \pi)^{2-\varepsilon}} K_{-1-\varepsilon}(m x), \quad D^{(3)}(x, m)=\frac{(x / m)^{2+\varepsilon}}{48(2 \pi)^{2-\varepsilon}} K_{-2-\varepsilon}(m x)
$$

Using the expansions

$$
K_{-1-\varepsilon}(\xi)=K_{1}(\xi)+\frac{\varepsilon}{\xi} K_{0}(\xi)+O\left(\varepsilon^{2}\right), \quad K_{-2-\varepsilon}(\xi)=K_{2}(\xi)+\frac{2 \varepsilon}{\xi} K_{1}(\xi)+\frac{2 \varepsilon}{\xi^{2}} K_{0}(\xi)+O\left(\varepsilon^{2}\right)
$$

we obtain

$$
\begin{aligned}
& B_{N}(0,0,2,3,3,4)=\frac{2^{-6-2 \varepsilon}}{3(\Gamma(1+\varepsilon))^{2} \Gamma(2-\varepsilon)} \int_{0}^{\infty} K_{-\varepsilon}(\xi)\left(K_{-1-\varepsilon}(\xi)\right)^{2} K_{-2-\varepsilon}(\xi) \xi^{7+2 \varepsilon} d \xi \\
& =\frac{1+\varepsilon}{192} \int_{0}^{\infty} K_{0}(\xi)\left(K_{1}(\xi)\right)^{2} K_{2}(\xi) \xi^{7} d \xi+\frac{\varepsilon}{96} \int_{0}^{\infty} K_{0}(\xi)\left(K_{1}(\xi)\right)^{2} K_{2}(\xi) \xi^{6} d \xi \\
& \quad+\frac{\varepsilon}{96} \int_{0}^{\infty} K_{0}(\xi)\left(K_{1}(\xi)\right)^{3} \xi^{6} d \xi+\frac{\varepsilon}{96} \int_{0}^{\infty}\left(K_{0}(\xi)\right)^{2}\left(K_{1}(\xi)\right)^{2} \xi^{5} d \xi \\
& \quad+\frac{\varepsilon}{96} \int_{0}^{\infty} K_{0}(\xi)\left(K_{1}(\xi)\right)^{2} K_{2}(\xi) \xi^{7} \ln \left(e^{\gamma_{E}} \xi / 2\right) d \xi
\end{aligned}
$$




\subsection{The reduction procedure}

Especially in the last expression given by Eq. (198) there are many different integrals which differ from the basis $L_{4}(r)$ and $L_{4}^{l}(r)$. However, the integrands can be reduced to integrands involving $K_{0}(\xi)$ and $K_{1}(\xi)$ only by using the relation

$$
K_{n}(\xi)=2 \frac{n-1}{\xi} K_{n-1}(\xi)+K_{n-2}(\xi) .
$$

After the first step, namely the expansion of Bessel functions for non-integer indices, the above relation establishes the second step in our reduction procedure. Finally, we use

$$
\begin{aligned}
\frac{d}{d \xi} K_{0}(\xi) & =-K_{1}(\xi) \quad \text { and } \\
\frac{d}{d \xi} K_{1}(\xi) & =-\frac{1}{2}\left(K_{0}(\xi)+K_{2}(\xi)\right)=-K_{0}(\xi)-\frac{1}{\xi} K_{1}(\xi)
\end{aligned}
$$

to perform the third and last step. For instance one has

$$
\begin{aligned}
L_{4}^{(1)}(r) & =\int_{0}^{\infty}\left(K_{0}(\xi)\right)^{3} K_{1}(\xi) \xi^{r} d \xi=-\int_{0}^{\infty}\left(K_{0}(\xi)\right)^{3} \frac{d K_{0}(\xi)}{d \xi} \xi^{r} d \xi= \\
& =-\left[K_{0}(\xi)\left(K_{0}(\xi)\right)^{3} \xi^{r}\right]_{0}^{\infty}+\int_{0}^{\infty} K_{0}(\xi) \frac{d}{d \xi}\left(K_{0}(\xi)\right)^{3} \xi^{r} d \xi= \\
& =3 \int_{0}^{\infty} K_{0}(\xi)\left(K_{0}(\xi)\right)^{2} \frac{d K_{0}(\xi)}{d \xi} \xi^{r} d \xi+r \int_{0}^{\infty} K_{0}(\xi)\left(K_{0}(\xi)\right)^{3} \xi^{r-1} d \xi= \\
& =-3 \int_{0}^{\infty}\left(K_{0}(\xi)\right)^{3} K_{1}(\xi) \xi^{r} d \xi+r \int_{0}^{\infty}\left(K_{0}(\xi)\right)^{4} \xi^{r-1} d \xi
\end{aligned}
$$

and therefore

$$
L_{4}^{(1)}(r)=\int_{0}^{\infty}\left(K_{0}(\xi)\right)^{3} K_{1}(\xi) \xi^{r} d \xi=\frac{r}{4} \int_{0}^{\infty}\left(K_{0}(\xi)\right)^{4} \xi^{r-1} d \xi=\frac{r}{4} L_{4}(r-1) .
$$

The reduction formulas are given in general by

$$
\begin{aligned}
L_{n}^{(m)}(r) & =\frac{1}{n-m+1}\left((r-m+1) L_{n}^{(m-1)}(r-1)-(m-1) L_{n}^{(m-2)}(r)\right) \\
L_{n}^{l(m)}(r) & =\frac{1}{n-m+1}\left((r-m+1) L_{n}^{l(m-1)}(r-1)+L_{n}^{(m-1)}(r-1)-(m-1) L_{n}^{l(m-2)}(r)\right)
\end{aligned}
$$

and are coded in MATHEMATICA in order to automatically reduce to the master integrals [39]. After executing the second and third step of the recursion the results for the above examples read

$$
\begin{aligned}
& B_{N}(0,0,2,2,2,2)=2(1+\varepsilon) L_{4}(3)+4 \varepsilon L_{4}^{l}(3)+O\left(\varepsilon^{2}\right), \\
& B_{N}(0,0,2,2,2,1)=2 L_{4}(1)+4 \varepsilon L_{4}^{l}(1)+O\left(\varepsilon^{2}\right), \\
& B_{N}(0,0,2,3,3,4)=\frac{1}{36} L_{4}(3)-\frac{1}{144} L_{4}(5)-\frac{1}{576} L_{4}(7)+O(\varepsilon) .
\end{aligned}
$$


Let us concentrate on the $O\left(\varepsilon^{0}\right)$ terms. By matching our numerical results to the results obtained by using the RECURSOR package [136],

$$
\begin{aligned}
B_{N}(0,0,2,2,2,2) & =-\frac{3}{8}+\frac{7}{16} \zeta(3)+O(\varepsilon), \\
B_{N}(0,0,2,2,2,1) & =\frac{7}{4} \zeta(3)+O(\varepsilon), \\
B_{N}(0,0,2,3,3,4) & =\frac{1}{576}+O(\varepsilon)
\end{aligned}
$$

we can determine the master integrals $L_{4}(r)$ for a few values of its argument. One has

$$
L_{4}(3)=-\frac{3}{16}+\frac{7}{32} \zeta(3), \quad L_{4}(1)=\frac{7}{8} \zeta(3), \quad 16 L_{4}(3)-2 L_{4}(5)-L_{4}(7)=1 .
$$

In particular the last relation is interesting because it implies a sum rule for the weighted product of three Bessel functions, namely

$$
\int_{0}^{\infty} K_{0}(\xi)\left(K_{1}(\xi)\right)^{2} K_{2}(\xi) \xi^{7} d \xi=\frac{1}{3}
$$

This surprising identity has been cross-checked by numerical integration. Note that for the $O\left(\varepsilon^{0}\right)$ contribution only odd values of $r$ appear as arguments in the master integral $L_{4}(r)$.

\subsection{Laplace-type asymptotics}

The two master integrals Eq. (184) are the basic set for the reduction procedure. A first estimate of the numerical magnitude of these integrals can be easily inferred from the asymptotic expansion of the integrals at large $q$,

$$
\begin{aligned}
& L_{4}(q)=\frac{\pi^{2} \Gamma(2 q)}{4^{2 q+1}}\left(1-\frac{1}{q-1 / 2}+O\left(1 / q^{2}\right)\right) \\
& L_{4}^{l}(q)=\frac{\pi^{2} \Gamma(2 q)}{4^{2 q+1}}\left(\Psi(2 q)+\gamma_{E}-3 \ln 2\right)\left(1-\frac{1}{q-1 / 2}+O\left(1 / q^{2}\right)\right)
\end{aligned}
$$

where $\Psi(x)=\Gamma^{\prime}(x) / \Gamma(x)$ is the logarithmic derivative of the $\Gamma$-function. The direct configuration space representation is the most convenient for numerical evaluation. To see this, we modify the last term of the integrals $L_{4}(q)$ and $L_{4}^{l}(q)$ by introducing parameters $\kappa$ and $\kappa_{l}$, respectively. In doing this, we find that the relations

$$
\begin{gathered}
L_{4}(q)=\frac{\pi^{2} \Gamma(2 q)}{4^{2 q+1}}\left(1-\frac{1}{q+\kappa}\right), \\
L_{4}^{l}(q)=\frac{\pi^{2} \Gamma(2 q)}{4^{2 q+1}}\left(\Psi(2 q)+\gamma_{E}-3 \ln 2\right)\left(1-\frac{1}{q+\kappa_{l}}\right)
\end{gathered}
$$


with $\kappa=0.97\left(\kappa_{l}=1.17\right)$ gives good results for the basis rational (resp. log-type) integrals with an accuracy better than $1 \%$ for all values of the arguments $q>1(q>3)$. For $q>3(q>5)$ the relative accuracy is better than $10^{-3}$. The leading order asymptotic formulas for the basis log-type integrals are less precise for small $q$ than its analogue in the rational case because the integrand in Eq. (184) is not positive for log-type integrals. Obviously, an exact solution via recurrence relations in momentum space will be much more complicated than these simple asymptotic formulae. Because the cancellation of significant figures in the numerical evaluation of terms with different transcendental structure is a dominating feature of the momentum space recurrence procedure for large $q$, the use of asymptotic formulas is worth considering.

\subsection{Irreducible numerator: three-loop vacuum bubble case}

Loop integrals may have numerator factors which involve the loop momenta and cannot be expanded in terms of the denominator pole factors. In such a case one speaks of irreducible numerator factors. Momentum space techniques can run into problems when non-trivial numerator factors appear. Take for example the numerator factor $\left(k_{1} \cdot k_{2}\right)$ for a $n$-loop bubble with $n+1$ massive lines. For $n<3$ the momentum space integral can be reduced to scalar integrals. For $n=3$, however, the numerator factorintegral is no longer reducible [137]. The problem of irreducible numerator factors has a straightforward solution in configuration space by the integration-by-parts technique [141]. The starting expression involving the non-trivial numerator factor $\left(k_{1} \cdot k_{2}\right)$ (or any other scalar product of linear independent inner moments) corresponds to

$$
\tilde{\Pi}_{3}^{*}(0)=\int D(x, m)^{2}\left(\partial_{\mu} D(x, m)\right)\left(\partial^{\mu}(D(x, m))\right) d^{D} x
$$

where the asterix denotes the fact that we are dealing with a non-scalar master integral. Careful use of the integration-by-parts identities [149, 150,

$$
\int \partial_{\mu}(D(x, m) \cdots D(x, m)) d^{D} x=0, \quad \int \square(D(x, m) \cdots D(x, m)) d^{D} x=0
$$

leads to the result

$$
\int D(x, m)^{2}\left(\partial_{\mu} D(x, m)\right)\left(\partial^{\mu}(D(x, m))\right) d^{D} x=-\frac{1}{3} \int D(x, m)^{3} \square D(x, m) d^{D} x .
$$

For the last integral we have used $\left(-\square+m^{2}\right) D(x, m)=\delta(x)$ and end up with

$$
\int D(x, m)^{3} \square D(x, m) d^{D} x=m^{2} \int D(x, m)^{4} d^{D} x-D(0, m)^{3} .
$$

The value of $D(0, m)$ can be found for instance by integration in momentum space,

$$
D(0, m)=\frac{1}{(2 \pi)^{D}} \int \frac{d^{D} p}{m^{2}+p^{2}}=\frac{2 \pi^{D / 2}}{(2 \pi)^{D} \Gamma(D / 2)} \int_{0}^{\infty} \frac{p^{D-1} d p}{p^{2}+m^{2}}=\frac{m^{D-2}}{(4 \pi)^{D / 2}} \Gamma(1-D / 2) .
$$


In the end we obtain

$$
\tilde{\Pi}_{3}^{*}(0)=-\frac{m^{2}}{3} \tilde{\Pi}_{3}(0)+\frac{1}{3} D(0, m)^{3}
$$

where $\tilde{\Pi}_{3}(0)$ is the scalar three-loop sunrise-type bubble diagram without a numerator factor.

\subsection{Irreducible numerator: four-loop vacuum bubble case}

Next we consider a four-loop diagram which appears as a second independent master integral $V_{2}$ of the sunrise topology in the classification of Ref. [137]. In momentum space the second master integral $V_{2}$ has the additional numerator factor $\left(k_{1} \cdot k_{4}\right)^{2}$ as compared to the first scalar master integral $V_{1}$. The second master integral reads

$$
\tilde{\Pi}_{4}^{*}(0)=\int \frac{\left(k_{1} \cdot k_{4}\right)^{2}(2 \pi)^{-4 D} d^{D} k_{1} d^{D} k_{2} d^{D} k_{3} d^{D} k_{4}}{\left(m_{1}^{2}+k_{1}^{2}\right)\left(m_{2}^{2}+\left(k_{2}-k_{1}\right)^{2}\right)\left(m_{3}^{2}+\left(k_{3}-k_{2}\right)^{2}\right)\left(m_{4}^{2}+\left(k_{4}-k_{3}\right)^{2}\right)\left(m_{5}^{2}+k_{4}^{2}\right)} .
$$

Turning again to the equal mass case and using the configuration space representation, this integral can be written as

$$
\tilde{\Pi}_{4}^{*}(0)=\int D(x, m)^{3}\left(\partial_{\mu} \partial_{\nu} D(x, m)\right)\left(\partial_{\mu} \partial_{\nu} D(x, m)\right) d^{D} x .
$$

It is apparent that by using integration-by-parts techniques this integral cannot be reduced to scalar integrals and/or integrals containing d'Alembertians. The easiest way to evaluate such an integral is to compute the derivatives directly. This is done with the help of the relation

$$
\frac{1}{z} \frac{d}{d z}\left(z^{-\nu} K_{\nu}(z)\right)=-\left(z^{-\nu-1} K_{\nu+1}(z)\right)
$$

(cf. Eq. (A12)). Eq.(219) can be iterated and gives results for arbitrary high order derivatives of Bessel functions $K_{\lambda}(z)$ in terms of the same class of Bessel functions with shifted indices and powers in $z$. For the first derivative we obtain

$$
\partial_{\mu} D(x, m)=-x_{\mu} \frac{m^{2 \lambda+2}}{(2 \pi)^{\lambda+1}} \frac{K_{\lambda+1}(m x)}{(m x)^{\lambda+1}} .
$$

Since the resulting analytical expression for a given line of the diagram lies in the same class as the original line, the procedure of evaluating the integral is similar to the usual one. However, we cannot use the second derivative

$$
\partial_{\mu} \partial_{\nu} D(x, m)=\frac{m^{2 \lambda+2}}{(2 \pi)^{\lambda+1}(m x)^{\lambda+1}}\left(g_{\mu \nu} K_{\lambda+2}(m x)-\frac{x_{\mu} x_{\nu}}{x^{2}} K_{\lambda+1}(m x)\right)
$$

directly under the integration sign. The reason is that the propagator has to be regarded as a distribution. There is a $\delta$-function singularity at the origin which is not taken into account in Eq. (221). Indeed, contracting the indices $\mu$ an $\nu$ in Eq. (221) one obtains

$$
\partial_{\mu} \partial^{\mu} D(x, m)=m^{2} D(x, m)
$$


while the correct equation for the propagator reads $\left(-\partial^{2}+m^{2}\right) D(x, m)=\delta(x)$. Thus, a straightforward evaluation of derivatives is valid only for $x \neq 0$. The behaviour at the origin $(x=0)$ requires special consideration. In practice, to treat this case one should not use higher order derivatives but stay at the level of the first derivative.

In order to deal with this case we introduce another master integral

$$
\tilde{\Pi}_{4}^{* *}(0)=\int D(x, m) \partial_{\mu} D(x, m) \partial_{\nu} D(x, m) \partial^{\mu} D(x, m) \partial^{\nu} D(x, m) d^{D} x .
$$

The relation between the two master integrals $\Pi_{4}^{*}(0)$ and $\Pi_{4}^{* *}(0)$ is found to be

$$
\tilde{\Pi}_{4}^{*}(0)=3 \tilde{\Pi}_{4}^{* *}(0)-\frac{1}{8} m^{4} \tilde{\Pi}_{4}(0)-\frac{7}{8} m^{2} D(0, m)^{4} .
$$

The quantity $\tilde{\Pi}_{4}^{* *}(0)$ can be calculated with the explicit use of first order derivatives within our technique. The analytical result for the pole part reads

$$
\mathcal{N}_{4} \tilde{\Pi}_{4}^{* *}(0)=m^{10}\left(-\frac{3}{8 \varepsilon^{4}}-\frac{277}{144 \varepsilon^{3}}-\frac{37837}{6912 \varepsilon^{2}}-\frac{4936643}{414720 \varepsilon}+O\left(\varepsilon^{0}\right)\right)
$$

$\left(\mathcal{N}_{4}=\left((4 \pi)^{2-\varepsilon} / \Gamma(1=\varepsilon)\right)^{4}\right.$, cf. Eq. (500) $)$ and the $\varepsilon$-expansion in the form

$$
\begin{aligned}
\mathcal{N}_{4} \tilde{\Pi}_{4}^{* *}(0)= & m^{10}\left(-0.375 \varepsilon^{-4}-1.923611 \varepsilon^{-3}-5.474103 \varepsilon^{-2}-11.90356 \varepsilon^{-1}\right. \\
& \left.-27.99303-104.5384 \varepsilon-663.6123 \varepsilon^{2}-3703.241 \varepsilon^{3}+O\left(\varepsilon^{4}\right)\right) .
\end{aligned}
$$

Since the results for $\tilde{\Pi}_{4}(0), \tilde{\Pi}_{4}^{* *}(0)$, and $D(0, m)^{4}$ are known, Eq. (224) can be used to obtain the final result for the original integral,

$$
\begin{aligned}
\mathcal{N}_{4} \tilde{\Pi}_{4}^{*}(0)= & m^{10}\left(-1.6875 \varepsilon^{-4}-7.8125 \varepsilon^{-3}-21.20964 \varepsilon^{-2}-44.76955 \varepsilon^{-1}\right. \\
& \left.-97.07652-290.9234 \varepsilon-1719.809 \varepsilon^{2}-8934.731 \varepsilon^{3}+O\left(\varepsilon^{4}\right)\right)
\end{aligned}
$$

which again verifies the result given in Ref. [137.

Differentiation of the massive propagator leads to expressions of a similar functional form which makes the configuration space technique a universal tool for calculating any master integral of the sunrise topology. This technique is also useful for finding master integrals. Indeed, new master integrals appear when there is a possibility to add new derivatives into the integrands which cannot be eventually removed by using the equations of motion or integrationby-parts recurrence relations. But once again: without explicit inclusion of the $\delta$-function only one derivative is allowed. Otherwise one misses tadpole parts of the result. Therefore, the new master integral should contain just one derivative for each line except for one line. This allows one to enumerate the number of non-scalar master integrals, i.e. master integrals including non-trivial numerator factors. For instance, in the five-loop case (six propagators) there will be only two non-scalar master integrals. 


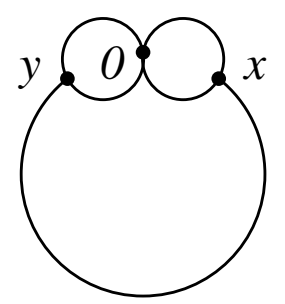

(a)

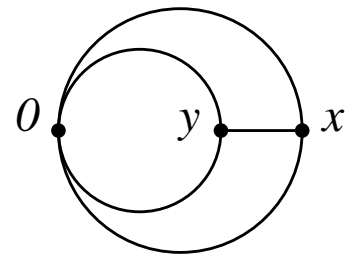

(b)

Figure 15: spectacle topology diagram in two different representations (a) (left hand side) and (b) (right hand side). The configurations space points $0, x$ and $y$ are indicated.

\section{Generalization: Still within reach}

Apart from the diagrams with sunrise-type topology treated in the previous sections there are more involved topologies close to the sunset-type topology that still allow for quite a simple numerical evaluation. We conclude this report by discussing several such cases which are still in reach of the methods presented here.

\subsection{Generalization to the spectacle topology}

In this subsection we give a formula for a more general topology which occurs when one propagator is removed from an initial three-loop, tetrahedron-shaped bubble diagram. In the original classification of Ref. 108 these diagrams are class $E$ diagrams belonging to the spectacle topology (see Fig. 15). The formulas obtained in this subsection are efficient for the numerical integration of diagrams of the spectacle topology. We have, however, not been able to find an analytical solution to the problem. The main obstacle of generalizing the configuration space technique to a general multi-loop diagram is the angular integration. The configuration space technique proved to be rather successful for general diagrams in the massless case [111] but it brings no essential simplification in the general massive case (see e.g. Ref. 25]). However, for special configurations the angular integration can be explicitly performed with a reasonably simple integrand left for the radial integration. Diagrams of the spectacle topology such as the ones shown in Fig. [15] are examples of such a configuration.

The configuration space expression of a spectacle topology diagram can be written in a form suitable for our purpose (see Fig. [15)(a)),

$$
\int D(x-y, m) D(x, m)^{2} d^{D} x D(y, m)^{2} d^{D} y .
$$

The key relation which leads to the simplification of the configuration space integral with spectacle topology is an addition theorem for Bessel functions in Eq. (A17), allowing to perform 
the integration over the relative angle in the propagator $D(x-y, m)$. Using Eq. (A17) for $Z=H^{+}$and substituting $m=e^{i \pi / 2}$, we can perform an analytic continuation in order to obtain a relation for the modified Bessel functions $K$ and $I$,

$$
\frac{K_{\lambda}(R)}{R^{\lambda}}=2^{\lambda} \Gamma(\lambda) \sum_{k=0}^{\infty}(\lambda+k) \frac{I_{\lambda+k}(\rho)}{\rho^{\lambda}} \frac{K_{\lambda+k}(r)}{r^{\lambda}} C_{k}^{\lambda}(\cos \varphi)
$$

where we have $R=\sqrt{r^{2}+\rho^{2}-2 r \rho \cos \varphi}$,

$$
\begin{array}{rlr}
K_{\lambda}(z) & =\frac{i \pi}{2} e^{\pi \lambda i / 2} H_{\lambda}^{+}(i z), \\
I_{\lambda}(z) & =e^{-\pi \lambda i / 2} J_{\lambda}\left(e^{\pi i / 2} z\right) \quad \text { for }-\pi<\arg z \leq \frac{\pi}{2}, \\
I_{\lambda}(z) & =e^{3 \pi \lambda i / 2} J_{\lambda}\left(e^{-3 \pi i / 2} z\right) \quad \text { for } \frac{\pi}{2}<\arg z \leq \pi .
\end{array}
$$

Using the orthogonality relations for Gegenbauer polynomials in Eq. (B33), the sum disappears after integration over the relative angle and only one term contributes. We obtain

$$
\begin{aligned}
\int \frac{K_{\lambda}(R)}{R^{\lambda}} d \Omega_{\rho} & =2^{\lambda} \Gamma(\lambda) \sum_{k=0}^{\infty}(\lambda+k) \frac{I_{\lambda+k}(\rho)}{\rho^{\lambda}} \frac{K_{\lambda+k}(r)}{r^{\lambda}} \int C_{k}^{\lambda}(\cos \varphi) d \Omega_{\varphi} \\
& =2^{\lambda} \Gamma(\lambda) \lambda \frac{I_{\lambda}(\rho)}{\rho^{\lambda}} \frac{K_{\lambda}(r)}{r^{\lambda}} \frac{2 \pi^{\lambda+1}}{\Gamma(\lambda+1)} C_{0}^{\lambda}(1) \\
& =(2 \pi)^{\lambda+1} \frac{I_{\lambda}(\rho)}{\rho^{\lambda}} \frac{K_{\lambda}(r)}{r^{\lambda}}, \quad r>\rho,
\end{aligned}
$$

where the first equality is a consequence of the orthogonality relation with the trivial factor $C_{0}^{\lambda}(1)=1$. This result allows one to write down an expression for any spectacle-type diagram in the form of a two-fold integral with a simple integration measure,

$$
\begin{aligned}
& \int_{0}^{\infty} D(x, m)^{2} x^{2 \lambda+1} d x \int_{0}^{\infty} D(y, m)^{2} y^{2 \lambda+1} d y \times \\
& \left(\frac{K_{\lambda}(x)}{x^{\lambda}} \frac{I_{\lambda}(y)}{y^{\lambda}} \theta(x-y)+\frac{K_{\lambda}(y)}{y^{\lambda}} \frac{I_{\lambda}(x)}{x^{\lambda}} \theta(y-x)\right) \\
= & \int_{0}^{\infty} D(x, m)^{2} I_{\lambda}(x) x^{\lambda+1} d x \int_{x}^{\infty} D(y, m)^{2} K_{\lambda}(y) y^{\lambda+1} d y
\end{aligned}
$$

where $\theta(x)$ is the standard step-function distribution [39].

Note that the integration measure $D(x, m)^{2} x^{2 \lambda+1} d x$ allows one to perform the integration by using efficient integration routines for numerical evaluation. The form of the weight function is close to $e^{-a x} x^{\alpha}$ which suggests the use of Laguerre polynomials

$$
L_{n}(x)=\frac{e^{x}}{n !} \frac{d^{n}}{d x^{n}}\left(x^{n} e^{-x}\right)
$$

as a convenient choice within the Gaussian numerical integration method. In fact, any modified propagator (with any power of the denominator) can be used as a factor in the integration 
measure $D(x, m)^{2} x^{2 \lambda+1} d x$. This fact makes the representation universal and useful for the case of higher powers of denominators of the lines associated with pairs $(x, 0)$ and $(y, 0)$ of spacetime points. If the angular structure of the diagram is preserved, the generalization to higher loops in the expressions for the radial measures is straightforward.

\subsection{Example for the spectacle diagram}

To show how this technique works, we give an example of the evaluation of a spectacle diagram. Consider an integer dimension space-time which, without loss of generality, can be chosen to be two-dimensional (an odd number of dimensions is trivial because, as we have seen earlier, the propagators degenerate to simple exponentials). The spectacle-type three-loop diagram can be obtained in a closed form. Indeed, in momentum space representation we have

$$
S(M)=\int \frac{\tilde{\Pi}(p)^{2}}{p^{2}+M^{2}} d^{2} p
$$

for the basic spectacle diagram $S$ with $\tilde{\Pi}(p)$ and the mass $M$ of the connecting propagator kept different. After the substitutions $p=2 m \sinh (\xi / 2)$ and $t=e^{-\xi}$ we obtain

$$
S(M)=\frac{1}{2 \pi m^{4}} \int_{0}^{1} \frac{t \ln ^{2} t d t}{\left(1-t^{2}\right)\left(1-2 \gamma t+t^{2}\right)}
$$

where $\gamma=1-M^{2} / 2 m^{2}$. Performing the integration, we finally obtain

$$
S(M)=\frac{f\left(t_{1}\right)-f\left(t_{2}\right)}{t_{1}-t_{2}}
$$

with $t_{1,2}=\gamma \pm \sqrt{\gamma^{2}-1}$ and

$$
f(t)=\frac{8 t \mathrm{Li}_{3}(1 / t)-(t+7) \zeta(3)}{8 \pi m^{4}\left(t^{2}-1\right)} .
$$

$\mathrm{Li}_{3}(z)$ is the trilogarithm function

$$
\operatorname{Li}_{3}(z)=\sum_{k=1}^{\infty} \frac{z^{k}}{k^{3}}, \quad|z|<1 .
$$

For $M=2 m$ (i.e. $\gamma=-1$ ) the integral in Eq. (235) simplifies and one finds a simple answer in terms of the (for the present context) standard transcendental numbers $\ln 2$ and $\zeta(3)$,

$$
S(2 m)=\frac{1}{4 \pi m^{4}}\left(\frac{7}{8} \zeta(3)-\ln 2\right) .
$$

For the equal mass case $M=m$ we obtain a result in terms of Clausen's trilogarithm $\mathrm{Cl}_{3}(2 \pi / 3)$. As one can conclude from these results, the conjugate pair of the sixth order roots of unity, 
$\exp ( \pm 2 \pi i / 3)$ play an important role in this case again in accordance with the general analysis in [110]. The origin of the appearance of the sixth order roots of unity as the parameters of the analytical expressions of the diagrams lies in the mismatch of masses along the lines of the diagrams. However, the exceptional case $M=2 m$, where one line has twice the mass of the other lines (which results in the drastic simplification), also keeps us within the set of the sixth order roots of unity. The key parameter in this case is simply the natural number 1 which is definitely one of the sixth order roots of unity.

Turning to the configuration space representation in Eq. (232) we find

$$
\begin{aligned}
S(M)= & \int_{0}^{\infty} x K_{0}(m x)^{2} d x \int_{0}^{\infty} y K_{0}(m y)^{2} d y \times \\
& \left(K_{0}(M x) I_{0}(M y) \theta(x-y)+K_{0}(M y) I_{0}(M x) \theta(y-x)\right) .
\end{aligned}
$$

for the basic spectacle diagram. An explicit numerical integration of Eq. (240) shows coincidence with the analytical result in Eq. (236) which we have checked numerically for arbitrary values of $M$. In this example the analytical result has a rather simple form. This is not true if high powers of the denominators enter. Then the corresponding one-loop insertions are rather cumbersome and an explicit integration in configuration space is more convenient.

\subsection{About the occurrence of Clausen's dilogarithms}

We want to have a closer look at how the square of Clausen's dilogarithm $\mathrm{Cl}_{2}(\pi / 3)^{2}$ can emerge at the level of spectacle topology diagrams. As transcendental number, Clausen's dilogarithm $\mathrm{Cl}_{2}(\pi / 3)$ characterizes the analytical results for three-loop bubbles. Its presence was discovered in the impressive treatise of David Broadhurst on the role of the sixth order roots of unity for the transcendental structure of results for Feynman diagrams in quantum field theory [110].

We consider the spectacle diagram in the form shown in Fig. 15(b). Here the expression for the generalized middle line is a product of the one-loop propagator and the standard particle propagator. We express the one-loop propagator using a dispersion representation with the spectral density $\rho(s)$ and obtain

$$
\frac{1}{p^{2}+m^{2}} \tilde{\Pi}(p)=\frac{1}{p^{2}+m^{2}} \int_{4 m^{2}}^{\infty} \frac{\rho(s) d s}{s+p^{2}}=\int_{4 m^{2}}^{\infty} \frac{\rho(s) d s}{s-m^{2}}\left(\frac{1}{p^{2}+m^{2}}-\frac{1}{s+p^{2}}\right) .
$$

We take only the first term which is sufficient for obtaining the result we are aiming for. In this case the integral becomes independent of $p^{2}$ and can be considered separately. One has

$$
I=\frac{1}{p^{2}+m^{2}} \int_{4 m^{2}}^{\infty} \frac{\rho(s) d s}{s-m^{2}}
$$


which leads to the sunrise diagram after the two other line shown in Fig. 15(b) have been added with a normalization factor given by the integral. One factor $\mathrm{Cl}_{2}(\pi / 3)$ results from integrating the overall sunrise diagram which is composed of the propagator $\left(p^{2}+m^{2}\right)^{-1}$ from Eq. (242). The two other lines of the diagram are shown in Fig. 15(b). The second factor $\mathrm{Cl}_{2}(\pi / 3)$ has to be found in the normalization factor given by the integral in Eq. (242). Note that the very structure of this contribution - the square of a number which first appeared at the lower loop level suggests a hint for its search. It should emerge as an iteration of a lower order contribution in accordance with the iterative structure of the R-operation which provides a general framework for the analysis of multi-loop diagrams. The following consideration confirms this conjecture. Consider the quantity

$$
N=\int_{4 m^{2}}^{\infty} \frac{\rho(s) d s}{s-m^{2}}
$$

and take $\rho(s)$ to be the spectral density in D-dimensional space-time [38],

$$
\rho(s)=\frac{\left(s-4 m^{2}\right)^{\lambda-1 / 2}}{2^{4 \lambda+1} \pi^{\lambda+1 / 2} \Gamma(\lambda+1 / 2) \sqrt{s}}, \quad \sqrt{s}>2 m .
$$

Next consider the first order contribution of the expansion in $\varepsilon$ near the space-time dimension $D_{0}=2$. The expansion in $\lambda=\varepsilon$ results in

$$
\frac{\left(s-4 m^{2}\right)^{\varepsilon-1 / 2}}{\mu^{2 \varepsilon} \sqrt{s}}=\frac{1}{\sqrt{s\left(s-4 m^{2}\right)}}\left(1+\varepsilon \ln \left(\frac{s-4 m^{2}}{\mu^{2}}\right)+O\left(\varepsilon^{2}\right)\right) .
$$

Therefore, the relevant first order term in $\varepsilon$ reads

$$
\Delta_{\varepsilon} \rho(s)=\frac{\ln \left(\left(s-4 m^{2}\right) / m^{2}\right)}{2 \pi \sqrt{s\left(s-4 m^{2}\right)}}
$$

where $\mu=m$ has been chosen for convenience. Next we change variables according to

$$
\sqrt{s}=2 m \cosh (\xi / 2), \quad t=e^{-\xi}
$$

to obtain

$$
\Delta_{\varepsilon} \rho\left(4 m^{2} \cosh ^{2}(\xi / 2)\right)=\frac{(-\ln t+2 \ln (1-t)) t}{2 \pi m^{2}\left(1-t^{2}\right)} .
$$

For the quantity in question we find

$$
\Delta_{\varepsilon} N=\int_{4 m^{2}}^{\infty} \frac{\Delta_{\varepsilon} \rho(s) d s}{s-m^{2}}=\frac{1}{2 \pi m^{2}} \int_{0}^{1} \frac{(-\ln t+2 \ln (1-t)) d t}{1+t+t^{2}} .
$$

The roots of the denominator of the integrand in Eq. (249) are now given by $t_{3,4}=\exp ( \pm 2 \pi i / 3)$ which again is a conjugate pair of the sixth order roots of unity. After integrating this equation 
we readily find

$$
\begin{aligned}
\Delta_{\varepsilon} N & =-\frac{1}{2 \pi m^{2} \sqrt{3}}\left(-\frac{\pi}{3} \ln 3+\operatorname{Im}\left(\operatorname{Li}_{2}\left(e^{2 i \pi / 3}\right)-\operatorname{Li}_{2}\left(e^{-2 i \pi / 3}\right)\right)\right) \\
& =-\frac{1}{\pi m^{2} \sqrt{3}}\left(\mathrm{Cl}_{2}\left(\frac{2 \pi}{3}\right)-\frac{\pi}{6} \ln 3\right) .
\end{aligned}
$$

Using the relation

$$
\mathrm{Cl}_{2}\left(\frac{2 \pi}{3}\right)=\frac{2}{3} \mathrm{Cl}_{2}\left(\frac{\pi}{3}\right)
$$

we finally obtain

$$
\Delta_{\varepsilon} N=-\frac{2}{3 \pi m^{2} \sqrt{3}}\left(\mathrm{Cl}_{2}\left(\frac{\pi}{3}\right)-\frac{\pi}{4} \ln 3\right) .
$$

Therefore, in the first order of the $\varepsilon$ expansion of the spectacle diagram we indeed found the remarkable contribution proportional to $\mathrm{Cl}_{2}(\pi / 3)^{2}$. In our calculation it emerges naturally as the iteration of the lower order term [47]. Originally, the presence of this contribution has been conjectured and confirmed in [110] by direct numerical computation of the finite part of the general three-loop bubble in four-dimensional space-time.

\subsection{The insertion of massless irreducible loops}

One can consider a sunrise-type diagram with one or more irreducible loops by which we mean a generalized line more complicated than an ordinary standard propagator or a (large) power of them. Consider, for instance, the replacement of a line by a subdiagram of fish-type topology as shown in Fig. 16. The configuration space technique leads to a numerical solution in this case because we can replace one of the propagator factors $D(x, m)$ by the two-point propagator in the irreducible subdiagram, using the fact that

$$
\Pi(x)=\int D(x, \sqrt{s}) \rho(s) d s
$$

where $\rho(s)$ is the spectral density of the subdiagram. If the subdiagram is a massless fish-type diagram, the spectral density is given by

$$
\rho(s)=6 \zeta(3) \delta(s)
$$

The known result in momentum space can be restored by using the dispersion relation (20). On the other hand, we obtain

$$
D(x)=6 \zeta(3) \int D(x, \sqrt{s}) \delta(s) d s=6 \zeta(3) D(x, 0)
$$

which up to a factor is the usual massless propagator. 


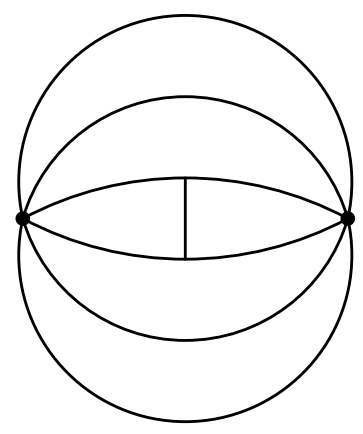

Figure 16: Irreducible loop replacing one of the propagators

\subsection{The insertion of massive irreducible loops}

Insertions of massive irreducible loops were used analytically for the calculation of corrections to baryon correlators [54, 56, 58] and numerically for the mixing of heavy neutral mesons [71]. The massive insertion can be incorporated mainly because the two-loop diagrams are well analyzed with any mass configurations [193, 194, 195, 196]. For the semi-massive fish-type one-loop diagram with one massive and one massless line we can use

$$
\rho(s)=\frac{4}{(4 \pi)^{4} s}\left(\operatorname{Li}_{2}\left(\frac{m^{2}}{s}\right)+\frac{1}{2} \ln \left(1-\frac{m^{2}}{s}\right) \ln \left(\frac{m^{2}}{s}\right)\right) .
$$

But even in the case with two different masses a result for the spectral density is available,

$$
\rho(s)=\frac{-4}{(4 \pi)^{4} s}\left(\operatorname{Li}_{2}\left(x_{1}\right)+\operatorname{Li}_{2}\left(x_{2}\right)-\frac{1}{2} \ln \left(-x_{1}\right) \ln \left(1-x_{1}\right)-\frac{1}{2} \ln \left(-x_{2}\right) \ln \left(1-x_{2}\right)\right)
$$

where

$$
\begin{aligned}
& x_{1}=\frac{2 m_{1}^{2}}{m_{1}^{2}+m_{2}^{2}-s+\sqrt{\left(m_{1}^{2}+m_{2}^{2}-s\right)^{2}-4 m_{1}^{2} m_{2}^{2}}}, \\
& x_{2}=\frac{2 m_{2}^{2}}{m_{1}^{2}+m_{2}^{2}-s+\sqrt{\left(m_{1}^{2}+m_{2}^{2}-s\right)^{2}-4 m_{1}^{2} m_{2}^{2}}}
\end{aligned}
$$




\section{Summary and conclusion}

To conclude, we have presented a review of configuration space techniques for the calculation of sunrise-type diagrams. We have shown that the singular or pole parts of any sunrise-type diagram are calculable analytically in the simplest possible manner. For the finite parts we obtained one-dimensional integrals of well-known functions which is very convenient for numerical evaluation. We have presented many sample calculations of multi-loop sunrise-type diagrams and have compared them with momentum space results in the literature when available. We have found agreement in every case. The agreement provides for a mutual check of the results which have been derived using very different methods. In the asymptotic analysis have dealed with different expansions. We can conclude that any kind of expansions is given as an expansion in parameters of the propagators and thus leads to the expansion coefficients in a straightforward way. Finally, extensions to non-standard propagators and other exotic settings figured out to be feasible as well as some considerations for slightly different topologies. All in all, we can stress again that the benefit of configuration space techiques is the fact that there is 'almost' no integration in sunrise-type diagrams, as Eq. (66) shows.

\section{Acknowledgements}

We thank Kostja Chetyrkin for discussion, Robert Delbourgo for kind attention and enthusiasm in advertising $x$-space, Andrey Davydychev for communication and help in finding references, Andrey Grozin for providing us with the source code of RECURSOR, with which a part of the calculation was cross-checked, David Broadhurst for criticism and friendly remarks, and Giampiero Passarino for communication. SG thanks the Mainz xloops-GiNaC-group for permanent interest, valuable comments and numerical cross-checks. AAP thanks V.A. Matveev for encouragement, attention and support, and P. Baikov for illuminating discussions of the present status of the optimization of recurrence relations under study by him. This work was supported by the INTAS grant No. 03-51-4007. SG acknowledges support by the DFG as a guest scientist in Mainz, by the Estonian target financed project No. 0182647s04, and by the grant No. 6216 given by the Estonian Science Foundation. The work of AAP was supported in part by the Russian Fund for Basic Research under contract No. 03-02-17177 and by the grant NS-2184.2003.2. 


\section{A Properties of Bessel functions}

Since Bessel functions play a crucial role in our calculations we collect a number of definitions and properties of Bessel functions, mostly taken from the handbooks of Watson (1944) [133], Prudnikov, Brychkov and Marichev (1990) [134, and Gradshteyn and Ryshik (1994) [135.

\section{Bessel functions and Hankel functions}

Ordinary Bessel functions are solutions of the Bessel differential equation

$$
z^{2} \frac{d^{2} Z_{\nu}(z)}{d z^{2}}+z \frac{d Z_{\nu}(z)}{d z}+\left(z^{2}-\nu^{2}\right) Z_{\nu}(z)=0
$$

( $\nu$ need not be an integer). There are two classes of solutions $Z_{\nu}(z)$. Bessel functions of the first kind $J_{\nu}(z)$ are nonsingular at the origin $z=0$ while Bessel functions of the second kind $Y_{\nu}(z)$ are singular at $z=0$. Combining Bessel functions of both classes, we end up with Hankel functions

$$
H_{\nu}^{ \pm}(z)=J_{\nu}(z) \pm i Y_{\nu}(z)
$$

(instead of $H_{\nu}^{ \pm}(z)$ the notation $H_{\nu}^{(1)}=H_{\nu}^{+}$and $H_{\nu}^{(2)}=H_{\nu}^{-}$are quite common).

Bessel functions of the first kind have the series expansion

$$
J_{\nu}(z)=\left(\frac{z}{2}\right)^{\nu} \sum_{k=0}^{\infty} \frac{\left(-z^{2} / 4\right)^{k}}{k ! \Gamma(\nu+k+1)}=\frac{(z / 2)^{\nu}}{\Gamma(1+\nu)}\left(1-\frac{(z / 2)^{2}}{1+\nu}+\ldots\right) .
$$

Including a factor occuring in practical applications, we can also write

$$
\left(\frac{z}{2}\right)^{-\nu} J_{\nu}(z)=\sum_{k=0}^{\infty} \frac{\left(-z^{2} / 4\right)^{k}}{k ! \Gamma(\nu+k+1)}=\frac{1}{\Gamma(1+\nu)}\left(1-\frac{(z / 2)^{2}}{1+\nu}+\ldots\right) .
$$

$\Gamma(z)$ is Euler's Gamma function. Functional equations are of help in order to relate Bessel functions of different degree. They are given by

$$
z Z_{\nu-1}(z)+z Z_{\nu+1}(z)=2 \nu Z_{\nu}(z), \quad Z_{\nu-1}(z)-Z_{\nu+1}(z)=2 \frac{d}{d z} Z_{\nu}(z)
$$

where $Z$ is any of the Bessel functions $J, Y$, or $H^{ \pm}$. As a consequence we obtain

$$
\frac{d^{k}}{d z^{k}}\left(z^{-\nu} J_{\nu}(z)\right)=-z^{-\nu} J_{\nu+k}(z)
$$




\section{Modified Bessel functions}

We can continue Bessel functions into the complex plane, ending up with modified Bessel functions of the first kind $I_{\nu}(z)$ and modified Bessel functions of the second kind $K_{\nu}(z)$, sometimes also known as McDonald functions. $I_{\nu}(z)$ and $K_{\nu}(z)$ are solutions of the modified Bessel differential equation

$$
z^{2} \frac{d^{2} Z_{\nu}(z)}{d z^{2}}+z \frac{d Z_{\nu}(z)}{d z}-\left(z^{2}-\nu^{2}\right) Z_{\nu}(z)=0
$$

The modified Bessel function of the second kind

$$
K_{\nu}(z)=\frac{\pi}{2} \frac{I_{-\nu}(z)-I_{\nu}(z)}{\sin (\pi \nu)}, \quad \Gamma(\nu) \Gamma(1-\nu)=\frac{\pi}{\sin (\pi \nu)}
$$

can be expressed by the modified Bessel function of the first kind $I_{\nu}(z)$ with series expansion

$$
I_{\nu}(z)=\left(\frac{z}{2}\right)^{\nu} \sum_{k=0}^{\infty} \frac{\left(z^{2} / 4\right)^{k}}{k ! \Gamma(\nu+k+1)}
$$

Therefore, one has

$$
\left(\frac{z}{2}\right)^{\nu} K_{\nu}(z)=\frac{\Gamma(\nu)}{2}\left[1+\frac{1}{1-\nu}\left(\frac{z}{2}\right)^{2}-\frac{\Gamma(1-\nu)}{\Gamma(1+\nu)}\left(\frac{z}{2}\right)^{2 \nu}\right]+O\left(z^{4}, z^{2+2 \nu}\right) .
$$

For Eq. (A5) one can we derive functional equations for the modified Bessel functions,

$$
\begin{gathered}
z I_{\nu-1}(z)-z I_{\nu+1}(z)=2 \nu I_{\nu}(z), \quad I_{\nu-1}(z)+I_{\nu+1}(z)=2 \frac{d}{d z} I_{\nu}(z) \\
z K_{\nu-1}(z)-z K_{\nu+1}(z)=-2 \nu K_{\nu}(z), \quad K_{\nu-1}(z)+K_{\nu+1}(z)=-2 \frac{d}{d z} K_{\nu}(z),
\end{gathered}
$$

For the differentiation of the modified Bessel function including an appropriate factor we derive

$$
\frac{d}{d z}\left(z^{-\nu} K_{\nu}(z)\right)=-z^{-\nu} K_{\nu+1}(z)
$$

Bessel functions can also be differentiated with respect to their index, if this index is given by an integer. For $K_{\nu}(x)$ we for instance obtain

$$
\left[\frac{\partial K_{\nu}(z)}{\partial \nu}\right]_{\nu= \pm n}= \pm \frac{1}{2} n ! \sum_{k=0}^{n-1}\left(\frac{z}{2}\right)^{k-n} \frac{K_{k}(z)}{k !(n-k)}, \quad n \in\{0,1, \ldots\} .
$$

Finally, the modified Bessel function $K_{\nu}$ and the Hankel function $H_{\nu}^{+}$are related by

$$
K_{\nu}(z)=\frac{\pi i}{2} e^{i \nu \pi / 2} H_{\nu}^{+}(i z)
$$




\section{Asymptotic behaviour}

Asymptotic expansions determine the behaviour of the modified Bessel functions of the second

kind and the Hankel functions $H_{\nu}^{-}$when the arguments become large. For the two functions just related to each other we obtain

$$
K_{\nu, N}^{a s}(z)=\left(\frac{\pi}{2 z}\right)^{1 / 2} e^{-z}\left[\sum_{n=0}^{N-1} \frac{(\nu, n)}{(2 z)^{n}}+\theta \frac{(\nu, N)}{(2 z)^{N}}\right], \quad(\nu, n):=\frac{\Gamma(\nu+n-1 / 2)}{n ! \Gamma(\nu-n-1 / 2)}
$$

and

$$
H_{\nu, N}^{-a s}(i z)=\left(\frac{2}{\pi z}\right)^{1 / 2} e^{z+i \nu \pi / 2}\left[\sum_{n=0}^{N-1} \frac{(-1)^{n}(\nu, n)}{(2 z)^{n}}+\theta \frac{(-1)^{N}(\nu, N)}{(2 z)^{N}}\right]
$$

where $\theta \in[0,1]$ is chosen appropriately. For the asymptotic behaviour of $I_{\nu}(z)$ see Eq. (C16).

\section{Addition theorem}

The addition theorem for Bessel functions is given by

$$
\frac{Z_{\nu}(m R)}{R^{\nu}}=2^{\nu} m^{-\nu} \Gamma(\nu) \sum_{k=0}^{\infty}(\nu+k) \frac{J_{\nu+k}(m \rho)}{\rho^{\nu}} \frac{Z_{\nu+k}(m r)}{r^{\nu}} C_{k}^{\nu}(\cos \varphi)
$$

where $C_{k}^{\nu}$ are the Gegenbauer polynomials (cf. Appendix $\mathrm{B}$ ), $Z$ is any of the Bessel functions $J, Y$, or $H^{ \pm}, R=\sqrt{r^{2}+\rho^{2}-2 r \rho \cos \varphi}$, and $r>\rho$. For $r<\rho$ the arguments of the Bessel functions on the right hand side of Eq. (A17) should be interchanged.

\section{B Properties of Gegenbauer polynomials}

Gegenbauer polynomials can be generated using the characteristic polynomial

$$
\left(t^{2}-2 t x+1\right)^{-\nu}=\sum_{n=0}^{\infty} t^{n} C_{n}^{\nu}(x) .
$$

In particular, we have $C_{0}^{\nu}(x)=1, C_{1}^{\nu}(x)=2 \nu x$ and

$$
(n+1) C_{n+1}^{\nu}(x)=2(n+\nu) x C_{n}^{\nu}(x)-(n+2 \nu-1) C_{n-1}^{\nu}(x) .
$$

Gegenbauer polynomials obey the orthogonality relations 111

$$
\int C_{m}^{\nu}\left(\hat{x}_{1} \cdot \hat{x}_{2}\right) C_{n}^{\nu}\left(\hat{x}_{2} \cdot \hat{x}_{3}\right) d \Omega_{2}=\frac{2 \pi^{\nu+1}}{\Gamma(\nu+1)} \frac{\nu \delta_{m n}}{n+\nu} C_{n}^{\nu}\left(\hat{x}_{1} \cdot \hat{x}_{3}\right), \quad \int d \Omega_{2}=\frac{2 \pi^{\nu+1}}{\Gamma(\nu+1)}
$$

where $\hat{x}_{i}$ are unit four-vectors and $d \Omega_{i}$ is the angular part of the integration measure. Finally, we have

$$
C_{n}^{\nu}(1)=\frac{\Gamma(n+2 \nu)}{n ! \Gamma(2 \nu)} .
$$




\section{Cuts and discontinuities}

Functions which are continuous at least on parts of the real axis can be continued to the whole complex plane. However, if singularities occur on the real axis or elsewhere in the complex plane, this continuation need no longer be unique. Examples which occur in the context of our calculations are logarithms and polylogarithms but also powers with non-integer exponent and Bessel functions. We will deal with these special cases in this appendix.

\section{The case of logarithms}

For $x=0$ the function $\ln (x)$ has a non-removable singularity while for negative real values it is not defined. However, we can continue to the negative real axis by following a path which circumvents the origin. It makes a difference whether we turn around the origin in the mathematically positive or negative sense. If we for instance start at the real value $x$ and turn around in the positive sense to continue to a value at $-x$, we can parametrize this by writing $z=x e^{i \varphi}$ where $\varphi$ starts at $\varphi=0$ and increases until it reaches the negative real axis for $\varphi=\pi$. By doing so, we obtain

$$
\ln \left(x e^{+i \pi}\right)=\ln x+i \pi .
$$

However, we can reach the negative real axis as well by starting with $\varphi$ again but going to negative values, reaching the negative real axis for $\varphi=-\pi$. In this case we obtain

$$
\ln \left(x e^{-i \pi}\right)=\ln x-i \pi \text {. }
$$

Obviously, the value " $\ln (-x)$ " is not unique. Indeed, we can even think of the continuation to the complex plane as a sheet which winds up and rises each time we turn around the origin, the so-called Riemannian sheet. Nevertheless, for only one turn we can calculate the difference between the values. This quantity is known as discontinuity, and it can be calculated actually for every complex value $z$ except for singular points. The definition is given by

$$
\operatorname{Disc} f(z)=f\left(z e^{+i 0}\right)-f\left(z e^{-i 0}\right), \quad e^{ \pm i 0}=\lim _{\epsilon \rightarrow 0} e^{ \pm i \epsilon}, \epsilon>0
$$

where $z=0$ is the singular point of the function $f(z)$. If we apply this definition to the case of a logarithm with negative argument, we obtain

$$
\text { Disc } \ln (-x)=\ln \left(-x e^{+i 0}\right)-\ln \left(-x e^{-i 0}\right)=\ln \left(x e^{-i \pi}\right)-\ln \left(x e^{+i \pi}\right)=-2 \pi i \theta(x)
$$

where $\theta(x)$ is the step function $(\theta(x)=1$ for $x>0, \theta(x)=0$ for $x<0)$. Note that we have been careful in replacing $-x e^{ \pm i 0}$ by $x e^{\mp i \pi}$. The discontinuity of powers of logarithms can be 
calculated as well, for instance

$$
\begin{aligned}
\text { Disc } \ln ^{2}(-x) & =\ln ^{2}\left(x e^{-i \pi}\right)-\ln ^{2}\left(x e^{+i \pi}\right) \\
& =\left((\ln x-i \pi)^{2}-(\ln x+i \pi)^{2}\right) \theta(x)=-4 \pi i \ln x \theta(x) .
\end{aligned}
$$

For later convenience we divide the discontinuity by the factor $2 \pi i$ to obtain

$$
\frac{1}{2 \pi i} \text { Disc } \ln (-x)=-\theta(x), \quad \frac{1}{2 \pi i} \text { Disc } \ln ^{2}(-x)=-2 \ln x \theta(x), \quad \ldots
$$

\section{The case of polylogarithms}

Polylogarithms can be defined iteratively by

$$
\operatorname{Li}_{n}(x)=\int_{0}^{x} \frac{\operatorname{Li}_{n-1}\left(x^{\prime}\right)}{x^{\prime}} d x^{\prime}, \quad \operatorname{Li}_{1}(x)=-\ln (1-x)
$$

For all the polylogarithms a discontinuity occurs for $x>1$. We obtain

$$
\operatorname{Li}_{1}\left(x e^{ \pm i 0}\right)=-\ln \left(1-x e^{ \pm i 0}\right)=-\ln \left(1+x e^{\mp i \pi}\right)=(-\ln (x-1) \pm i \pi) \theta(x-1)
$$

where we have extracted a factor $e^{\mp i \pi}$ from the argument, and therefore

$$
\frac{1}{2 \pi i} \operatorname{Disc}_{\operatorname{Li}_{1}}(x)=\theta(x-1) \text {. }
$$

In order to calculate the discontinuity of $\operatorname{Li}_{2}(x)$, for $x>1$ we split the integral into two parts,

$$
\begin{aligned}
\operatorname{Li}_{2}\left(x e^{ \pm i 0}\right) & =-\int_{0}^{1} \frac{\ln \left(1-x^{\prime}\right)}{x^{\prime}} d x^{\prime}-\int_{1}^{x} \frac{\ln \left(1-x^{\prime}\right)}{x^{\prime}} d x^{\prime} \\
& =\operatorname{Li}_{2}(1)-\int_{1}^{x} \frac{\ln \left(x^{\prime}-1\right)}{x^{\prime}} d x \pm \pi i \int_{1}^{x} \frac{d x^{\prime}}{x^{\prime}}
\end{aligned}
$$

The real parts cancel out when taking the difference needed for the determination of the discontinuity. Contrary to this the imaginary parts add up and one obtains

$$
\begin{aligned}
\frac{1}{2 \pi i} \operatorname{Disc}_{2}(x) & =\frac{1}{2 \pi i} \int \frac{d x^{\prime}}{x^{\prime}} \operatorname{Disc} \operatorname{Li}_{1}\left(x^{\prime}\right)=\int_{1}^{x} \frac{d x^{\prime}}{x^{\prime}}=\ln x \theta(x-1), \quad \text { in the same way } \\
\frac{1}{2 \pi i} \operatorname{Disc~}_{L_{3}}(x) & =\frac{1}{2 \pi i} \int \frac{d x^{\prime}}{x^{\prime}} \operatorname{Disc~Li}_{2}\left(x^{\prime}\right)=\int_{1}^{x} \frac{d x^{\prime}}{x^{\prime}} \ln x^{\prime}=\frac{1}{2} \ln ^{2} x \theta(x-1), \quad \text { in general } \\
\frac{1}{2 \pi i} \operatorname{Disc~}_{n}(x) & =\frac{1}{n-1} \ln ^{n-1} x \theta(x-1)
\end{aligned}
$$




\section{The case of non-integer powers}

For powers with exponents close to integer values such as $(b-a x)^{-n}$ the calculation of the discontinuity is closely related to the calculation of the discontinuity of the logarithm because

$$
x^{\varepsilon}=1+\varepsilon \ln x+O\left(\varepsilon^{2}\right) .
$$

Similarly, the discontinuity of $(b-a x)^{-n}$ can be obtained in the more general case in closed form. In order to calculate the discontinuity of a power $(b-a x)^{-n}$ with non-integer exponent $-n$ (we include the minus sign for later convenience) we first have to ponder where the discontinuities can appear. Obviously, this is the case for $b-a x<0$. Taking $a>0$ and $b \geq 0$ which is the case for all applications in this report, we obtain $x>b / a$. Therefore, we obtain

$$
\begin{aligned}
& \operatorname{Disc}(b-a x)^{-n}=\left(b-a x^{+i 0}\right)^{-n}-\left(b-a x^{-i 0}\right)^{-n}=\left(b+a x e^{-i \pi}\right)^{-n}-\left(b+a x e^{+i \pi}\right)^{-n} \\
& =\left(e^{+i \pi n}(a x-b)^{-n}-e^{-i \pi n}(a x-b)^{-n}\right) \theta(a x-b)=2 i \sin (\pi n)(a x-b)^{-n} \theta(a x-b) .
\end{aligned}
$$

Using

$$
\sin (\pi n)=\frac{\pi}{\Gamma(n) \Gamma(1-n)}=-\sin (-\pi n)
$$

where $\Gamma(x)$ is Euler's Gamma function, we finally obtain

$$
\frac{1}{2 \pi i} \operatorname{Disc}(b-a x)^{-n}=\frac{(a x-b)^{-n} \theta(a x-b)}{\Gamma(n) \Gamma(1-n)}
$$

Note that for negative integer values of $n$ the discontinuity vanishes.

\section{The case of Bessel functions}

The final item about ambiguities found in the continuation into the complex plane we want to mention here is the so-called Stokes' phenomenon which occurs for the asymptotic expansion of Bessel functions and is mentioned already in the handbook of Watson [133. The asymptotic expansion of the modified Bessel function of the first kind reads

$$
\begin{aligned}
I_{\nu}(z)=\frac{e^{z}}{\sqrt{2 \pi z}} & \sum_{k=0}^{\infty} \frac{(-1)^{k}}{(2 z)^{k}} \frac{\Gamma(\nu+k+1 / 2)}{\Gamma(\nu-k+1 / 2) k !} \\
& +\frac{e^{-z \pm(\nu+1 / 2) \pi i}}{\sqrt{2 \pi z}} \sum_{k=0}^{\infty} \frac{1}{(2 z)^{k}} \frac{\Gamma(\nu+k+1 / 2)}{\Gamma(\nu-k+1 / 2) k !},
\end{aligned}
$$

where the plus sign is valid for $-\pi / 2<\arg z<3 \pi / 2$ while the minus sign has to be taken for $-3 \pi / 2<\arg z<\pi / 2$. The surprising fact is that there are two different asymptotic expansions available at every point of the complex plane, except for the arguments $\pm i \pi / 2$. Therefore, in continuing $I_{\nu}( \pm i x)=I_{\nu}\left(x e^{ \pm i \pi / 2}\right)$ to the complex plane, the choice is still unique. 


\section{Singular contributions up to four loops}

In the following we present complete results for the singular parts of sunrise-type diagrams with arbitrary masses up to four-loop order. The results are given in the $\overline{\mathrm{MS}}$-scheme in the Euclidean domain.

$$
\begin{aligned}
& \tilde{\Pi}_{1}^{s}\left(p, m_{1}, m_{2}\right)=\frac{1}{\varepsilon}, \\
& \tilde{\Pi}_{2}^{s}\left(p, m_{1}, m_{2}, m_{3}\right)=-\frac{1}{2 \varepsilon^{2}} \sum_{i} m_{i}^{2}-\frac{1}{4 \varepsilon}\left(p^{2}+2 \sum_{i} m_{i}^{2}\left(3-2 \ell_{i}\right)\right), \\
& \tilde{\Pi}_{3}^{s}\left(p, m_{1}, m_{2}, m_{3}, m_{4}\right)=\frac{1}{6 \varepsilon^{3}} \sum_{i \neq j} m_{i}^{2} m_{j}^{2}+\frac{1}{12 \varepsilon^{2}}\left(p^{2} \sum_{i} m_{i}^{2}-\sum_{i} m_{i}^{4}+2 \sum_{i \neq j} m_{i}^{2} m_{j}^{2}\left(4-3 \ell_{i}\right)\right)+ \\
& +\frac{1}{72 \varepsilon}\left(2 p^{4}+9 p^{2} \sum_{i} m_{i}^{2}\left(3-2 \ell_{i}\right)-9 \sum_{i} m_{i}^{4}\left(5-2 \ell_{i}\right)+6 \sum_{i \neq j} m_{i}^{2} m_{j}^{2}\left(20-24 \ell_{i}+3 \ell_{i}^{2}+6 \ell_{i} \ell_{j}\right)\right), \\
& \tilde{\Pi}_{4}^{s}\left(p, m_{1}, m_{2}, m_{3}, m_{4}, m_{5}\right)=-\frac{1}{24 \varepsilon^{4}} \sum_{i \neq j \neq k} m_{i}^{2} m_{j}^{2} m_{k}^{2}+ \\
& \quad-\frac{1}{48 \varepsilon^{3}}\left(p^{2} \sum_{i \neq j} m_{i}^{2} m_{j}^{2}-\sum_{i \neq j}\left(m_{i}^{4} m_{j}^{2}+m_{i}^{2} m_{j}^{4}\right)+2 \sum_{i \neq j \neq k} m_{i}^{2} m_{j}^{2} m_{k}^{2}\left(5-4 \ell_{i}\right)\right) \\
& \quad-\frac{1}{288 \varepsilon^{2}}\left(2 p^{4} \sum_{i} m_{i}^{2}-6 p^{2} \sum_{i} m_{i}^{4}+2 \sum_{i} m_{i}^{6}+3 p^{2} \sum_{i \neq j} m_{i}^{2} m_{j}^{2}\left(11-8 \ell_{i}\right)\right. \\
& \left.\quad-6 \sum_{i \neq j}\left(m_{i}^{4} m_{j}^{2}+m_{i}^{2} m_{j}^{4}\right)\left(7-4 \ell_{i}\right)+12 \sum_{i \neq j \neq k} m_{i}^{2} m_{j}^{2} m_{k}^{2}\left(15-20 \ell_{i}+2 \ell_{i}^{2}+6 \ell_{i} \ell_{j}\right)\right) \\
& \quad \frac{1}{1728 \varepsilon}\left(3 p^{6}+2 p^{4} \sum_{i} m_{i}^{2}\left(35-24 \ell_{i}\right)-18 p^{2} \sum_{i} m_{i}^{4}\left(21-8 \ell_{i}\right)+2 \sum_{i} m_{i}^{6}\left(77-24 \ell_{i}\right)\right. \\
& \quad+9 p^{2} \sum_{i \neq j} m_{i}^{2} m_{j}^{2}\left(71-88 \ell_{i}+8 \ell_{i}^{2}+24 \ell_{i} \ell_{j}\right)-216 \sum_{i \neq j}\left(m_{i}^{4} m_{j}^{2}-m_{i}^{2} m_{j}^{4}\right) \ell_{i} \\
& \quad-18 \sum_{i \neq j}\left(m_{i}^{4} m_{j}^{2}+m_{i}^{2} m_{j}^{4}\right)\left(49-56 \ell_{i}+4 \ell_{i}^{2}+12 \ell_{i} \ell_{j}\right) \\
& \left.\quad+24 \sum_{i \neq j \neq k} m_{i}^{2} m_{j}^{2} m_{k}^{2}\left(105-180 \ell_{i}+30 \ell_{i}^{2}+90 \ell_{i} \ell_{j}-2 \ell_{i}^{3}-18 \ell_{i}^{2} \ell_{j}-12 \ell_{i} \ell_{j} \ell_{k}\right)\right)
\end{aligned}
$$

where $\ell_{i}=\ln \left(m_{i}^{2} / \mu^{2}\right)$. The indices $i, j$, and $k$ run over all mass indices. One can check that the general results listed in this Appendix reproduce the equal mass results in the main text. 


\section{E Subtraction terms for the small $x$ singularities}

The leading singularity at small $x$ is given by the massless propagator of the form

$$
D(x, 0)=\frac{\Gamma(\lambda)}{4 \pi^{\lambda+1} x^{2 \lambda}} .
$$

The next order of the small $x$-expansion for the propagator $D(x, m)$ is explicitly given by

$$
D_{1}(x, 0)=\frac{1}{4 \pi^{\lambda+1} x^{2 \lambda}}\left(\left(\frac{x}{2}\right)^{2} \frac{\Gamma(\lambda)}{1-\lambda}-\left(\frac{x}{2}\right)^{2 \lambda} \frac{\Gamma(1-\lambda)}{\lambda}\right) .
$$

This term is suppressed relative to the first term by one power of $x^{2}$ at small $x$ in fourdimensional space-time (however, this is not the case for two-dimensional space-time with $\lambda=0)$. The term

$$
D_{2}(x, 0)=\frac{1}{4 \pi^{\lambda+1} x^{2 \lambda}}\left(\frac{x}{2}\right)^{2}\left(\left(\frac{x}{2}\right)^{2} \frac{\Gamma(\lambda)}{2(1-\lambda)(2-\lambda)}-\left(\frac{x}{2}\right)^{2 \lambda} \frac{\Gamma(1-\lambda)}{\lambda(\lambda+1)}\right)
$$

is further suppressed by one power of $x^{2}$ at small $x$. Therefore, the full subtraction of the three terms gives a rather smooth behaviour at small $x$ which is sufficient to obtain a regular integrand for the numerical integration.

\section{F Analytical results for the four-loop sunrise diagram}

In this appendix we present some more details of our calculations for the four-loop sunrise diagram. For the analytical evaluation we take the last two terms of the integrand from Eq. (78). One has to integrate a product of two Bessel functions multiplied with powers of $x$ which can be done analytically. The explicit expression for the $\varepsilon$-expansion of that part of the integral which is evaluated analytically reads

$$
\begin{aligned}
\tilde{\Pi}_{4}^{\text {ana }}(0)= & m^{6}\left\{-\frac{5}{2 \varepsilon^{4}}-\frac{35}{3 \varepsilon^{3}}-\frac{4565}{144 \varepsilon^{2}}-\frac{58345}{864 \varepsilon}\right. \\
- & \frac{1456940638037}{7779240000}-\frac{17099 \pi^{2}}{24}-\frac{3857 \pi^{4}}{10}+\frac{2525968 \zeta(3)}{105} \\
+ & \left(-\frac{55171475321621447}{1633640400000}+\frac{2457509 \pi^{2}}{144}-\frac{1292537 \pi^{4}}{175}\right. \\
& \left.+\frac{6752474831 \zeta(3)}{44100}+16530 \pi^{2} \zeta(3)+59508 \zeta(5)\right) \varepsilon \\
+ & \left(-\frac{10610679621089130529}{68612896800000}+\frac{92781949 \pi^{2}}{864}-\frac{4290113759 \pi^{4}}{110250}-\frac{22591 \pi^{6}}{14}\right.
\end{aligned}
$$




$$
\begin{aligned}
& \left.+\frac{952412727629 \zeta(3)}{9261000}+244476 \pi^{2} \zeta(3)-168606 \zeta(3)^{2}+\frac{32210272 \zeta(5)}{35}\right) \varepsilon^{2} \\
+ & -\frac{5963907632629558995931}{14408708328000000}+\frac{1325204033 \pi^{2}}{5184} \\
& -\frac{464379085699 \pi^{4}}{6615000}-\frac{48529231 \pi^{6}}{2205} \\
& -\frac{312138383154103 \zeta(3)}{277830000}+\frac{7285043 \pi^{2} \zeta(3)}{6}+43529 \pi^{4} \zeta(3)-\frac{238229084 \zeta(3)^{2}}{105} \\
& \left.\left.+\frac{13583011297 \zeta(5)}{2940}+247950 \pi^{2} \zeta(5)+1190160 \zeta(7)\right) \varepsilon^{3}+O(\varepsilon)^{4}\right\} .
\end{aligned}
$$

This expression shows the real complexity of the calculation which reveals itself in the structure of the results. The main feature is that the terms cannot be simultaneously simplified to all orders in $\varepsilon$. By a special choice of the normalization factor one can make the leading term and, in fact, even all pole terms simple, but then the higher order terms contain rather lengthy combinations of transcendental numbers that are not reducible in terms of standard quantities such as the Riemann $\zeta$-functions. Note also that the rational coefficients of transcendental numbers are very big and there is a huge numerical cancellation between the rational and transcendental parts of the answer (see also the discussion in Ref. [39]).

\section{G Integrand for the numerical integration}

For the numerical evaluation we take the first three terms of the integrand from Eq. (778). One has to integrate them numerically as there is a product of three or more Bessel functions which is too complicated to be done analytically. To find the $\varepsilon$-expansion of the integral one has to first expand the integrand in $\varepsilon$. The expression for the $\varepsilon$-expansion is quite lengthy. We therefore give explicit results only for $\varepsilon=0$. For this part the integrand for the numerical integration over $z=m x$ reads

$$
\begin{aligned}
\Pi_{4}^{\text {num }}(x)=m^{6}( & 66-108 l-144 l^{2}+192 l^{3}+\frac{384}{z^{6}}-\frac{384}{z^{4}}+\frac{768 l}{z^{4}}+\frac{24}{z^{2}}-\frac{480 l}{z^{2}}+\frac{576 l^{2}}{z^{2}} \\
& -\frac{111 z^{2}}{16}+\frac{147 l z^{2}}{2}-117 l^{2} z^{2}+24 l^{3} z^{2}+24 l^{4} z^{2}+-\frac{165 z^{4}}{32} \\
& +\frac{201 l z^{4}}{16}+\frac{9 l^{2} z^{4}}{2}-24 l^{3} z^{4}+12 l^{4} z^{4}+\frac{75 z^{6}}{512}-\frac{405 l z^{6}}{128}+\frac{531 l^{2} z^{6}}{64} \\
& -\frac{15 l^{3} z^{6}}{2}+\frac{9 l^{4} z^{6}}{4}+\frac{375 z^{8}}{2048}-\frac{825 l z^{8}}{1024}+\frac{315 l^{2} z^{8}}{256}-\frac{51 l^{3} z^{8}}{64}+\frac{3 l^{4} z^{8}}{16} \\
& \left.+\frac{1875 z^{10}}{131072}-\frac{375 l z^{10}}{8192}+\frac{225 l^{2} z^{10}}{4096}-\frac{15 l^{3} z^{10}}{512}+\frac{3 l^{4} z^{10}}{512}\right) K_{1}(z)
\end{aligned}
$$




$$
\begin{aligned}
+m^{6} & \left(\frac{-512}{z^{5}}+\frac{384}{z^{3}}-\frac{768 l}{z^{3}}+\frac{24}{z}+\frac{288 l}{z}-\frac{384 l^{2}}{z}-52 z+120 l z-64 l^{3} z\right. \\
& -\frac{15 z^{3}}{8}-21 l z^{3}+48 l^{2} z^{3}-24 l^{3} z^{3}+\frac{75 z^{5}}{32}-\frac{135 l z^{5}}{16}+9 l^{2} z^{5}-3 l^{3} z^{5} \\
& \left.+\frac{125 z^{7}}{512}-\frac{75 l z^{7}}{128}+\frac{15 l^{2} z^{7}}{32}-\frac{l^{3} z^{7}}{8}\right) K_{1}(z)^{2}+m^{6} \frac{128 K_{1}(z)^{5}}{z^{2}} .
\end{aligned}
$$

Here $l=\ln \left(e_{E}^{\gamma} z / 2\right), z=m x$, and $\gamma_{E}=-\Gamma^{\prime}(1)=0.577 \ldots$ is Euler's constant. As shown in Fig. 5, the plot of this function as well as the shapes of the corresponding functions in higher orders of $\varepsilon$ are very smooth and quite similar. The analytical expressions for higher orders in $\varepsilon$, however, become much longer. Note that the new functions $f_{n}(z)$ first appear at order $\varepsilon^{2}$.

The smoothness of the zeroth order integrand as shown in Eq. (G1) implies that the numerical integration is quite easy to execute. Because the integrand vanishes exponentially for large values of $z$ and has no singularities of the kind $z \ln z$ for small values of $z$, the integration can in principle range from 0 to $\infty$. However, for practical reasons we had to instruct Wolfram's Mathematica system for symbolic manipulations (which we used for all of our calculations presented here) that the integrand vanishes for $z=0$. On the other hand, the asymptotic expansion of the integrand together with the integration measure is dominated by the part

$$
\frac{6 \pi^{2} m^{2}}{512} z^{10} \ln ^{4}\left(e^{\gamma} z / 2\right) K_{1}(z) z^{3} d z \quad\left(K_{\lambda}(z) \rightarrow \sqrt{\frac{\pi}{2 z}} e^{-z} \text { for } z \rightarrow \infty\right)
$$

Integrated from $\Lambda$ to $\infty$, this part gives a contribution

$$
\frac{6 \pi^{2} m^{2}}{512} \Lambda^{25 / 2} \ln ^{4}\left(e^{\gamma} \Lambda / 2\right) e^{-\Lambda}
$$

and terms which are of subleading order. Therefore, the result can be well estimated by

$$
\tilde{\Pi}_{4}^{\text {num }}(0)=2 \pi^{2} \int_{0}^{\infty} \Pi_{4}^{\text {num }}(x) x^{3} d x \approx 2 \pi^{2} \int_{0}^{\Lambda} \Pi_{4}^{\text {num }}(x) x^{3} d x+\frac{6 \pi^{2} m^{2}}{512} \Lambda^{25 / 2} \ln ^{4}\left(e^{\gamma} \Lambda / 2\right) e^{-\Lambda}
$$

and $\Lambda$ can be adjusted to any desired precision.

A possibility to avoid any cutoff is to change the integration variable such that the interval $[0, \infty]$ is mapped onto $[0,1]$. Then the integration can be done numerically with the additional information that the integrand vanishes identically at both end points. Possible transformations of this kind are for instance $z=\ln (1 / t)$ or $z=(1-t) / t$ for $t \in[0,1]$. 


\section{References}

[1] P. Langacker (ed.), "Precision Tests of the Standard Electroweak Model", World Scientific, 1995.

[2] J.H. Kühn, "Expansion techniques in massive quark production: Results and applications", in Barcelona 1998, Radiative corrections: Application of quantum field theory to phenomenology, pp. 202-222 Report No. TTP-99-01 arXiv:hep-ph/9901330; Nucl. Phys. Proc. Suppl. 76 (1999) 21; J.H. Kühn and P.M. Zerwas, Adv. Ser. Direct. High Energy Phys. 15 (1998) 493

[3] W. Hollik, AIP Conf. Proc. 490 (1999) 10

[4] R. Barate et al. [ALEPH Collaboration], Phys. Lett. B495 (2000) 1; M. Acciarri et al. [L3 Collaboration], Phys. Lett. B495 (2000) 18

[5] W. Hollik and G. Duckeck, "Electroweak precision tests at LEP", in Springer tracts in modern physics, 162, Springer, Berlin (2000)

[6] H.N. Brown et al. [Muon g-2 Collaboration], Phys. Rev. Lett. 86 (2001) 2227;

A. Czarnecki and W.J. Marciano, Phys. Rev. D64 (2001) 013014;

J. R. Ellis, D.V. Nanopoulos and K.A. Olive, Phys. Lett. B508 (2001) 65;

F.J. Yndurain, "Disagreement between standard model and experiment for muon $g-2$ ?", Report No. FTUAM-01-04 arXiv:hep-ph/0102312

[7] M. Hayakawa and T. Kinoshita, "Comment on the sign of the pseudoscalar pole contribution to the muon $g-2$ ", Report No. KEK-TH-793 arXiv:hep-ph/0112102

[8] A.A. Pivovarov, Phys. Atom. Nucl. 66 (2003) 902 [Yad. Fiz. 66 (2003) 934]

[9] S. Groote, J.G. Körner and A.A. Pivovarov, Eur. Phys. J. C24 (2002) 393

[10] J.S. Schwinger, Phys. Rev. 73 (1948) 416

[11] T. Kinoshita, B. Nizic and Y. Okamoto, Phys. Rev. D41 (1990) 593

[12] T. Kinoshita and M. Nio, Phys. Rev. D70 (2004) 113001

[13] V.W. Hughes and T. Kinoshita, Rev. Mod. Phys. 71 (1999) S133

[14] P.J. Mohr and B.N. Taylor, Rev. Mod. Phys. 72 (2000) 351 
[15] K.G. Chetyrkin, A.L. Kataev and F.V. Tkachov, Phys. Lett. B85 (1979) 277

[16] S.G. Gorishnii, A.L. Kataev and S.A. Larin, Phys. Lett. B259 (1991) 144

[17] E. Braaten, S. Narison and A. Pich, Nucl. Phys. B373 (1992) 581

[18] A.E. Dorokhov, Int. J. Mod. Phys. A20 (2005) 631

[19] A.A. Pivovarov, Sov. J. Nucl. Phys. 54 (1991) 676 (1991), Z. Phys. C53 (1992) 461

[20] R. Coquereaux, Phys. Rev. D23 (1981) 2276

[21] V.I. Zakharov, Nucl. Phys. B385 (1992) 452

[22] N.V. Krasnikov and A.A. Pivovarov, Mod. Phys. Lett. A11 (1996) 835;

A.A. Penin and A.A. Pivovarov, Phys. Lett. B401 (1997) 294

[23] M. Beneke, "Renormalons" Phys. Rept. 317 (1999) 1

[24] A.G. Grozin, Nucl. Instrum. Meth. A502 (2003) 815

[25] E. Mendels, Nuovo Cim. A45 (1978) 87

[26] E. Mendels, J. Math. Phys. 43 (2002) 3011

[27] F.A. Berends, M. Buza, M. Böhm and R. Scharf, Z. Phys. C63 (1994) 227

[28] P. Mastrolia and E. Remiddi, Nucl. Phys. Proc. Suppl. 116 (2003) 412

[29] P. Mastrolia and E. Remiddi, Nucl. Phys. B657 (2003) 397

[30] H. Czyż, A. Grzelińska and R. Zabawa, Phys. Lett. B538 (2002) 52

[31] Y. Schröder, Nucl. Phys. Proc. Suppl. 116 (2003) 402

[32] P. Post and K. Schilcher, Phys. Rev. Lett. 79 (1997) 4088

[33] P. Post and J.B. Tausk, Mod. Phys. Lett. A11 (1996) 2115

[34] A.K. Rajantie, Nucl. Phys. B480 (1996) 729; B513 (1998) 761(E)

[35] F.A. Berends, A.I. Davydychev and N.I. Ussyukina, Phys. Lett. B426 (1998) 95

[36] J. Gasser and M.E. Sainio, Eur. Phys. J. C6 (1999) 297 
[37] S. Groote, J.G. Körner and A.A. Pivovarov, Phys. Lett. B443 (1998) 269

[38] S. Groote, J.G. Körner and A.A. Pivovarov, Nucl. Phys. B542 (1999) 515

[39] S. Groote, J.G. Körner and A.A. Pivovarov, Eur. Phys. J. C11 (1999) 279

[40] S. Groote and A.A. Pivovarov, Nucl. Phys. B580 (2000) 459

[41] R. Delbourgo and M.L. Roberts, J. Phys. A36 (2003) 1719

[42] M. Caffo, H. Czyż and E. Remiddi, Nucl. Phys. Proc. Suppl. 116 (2003) 422

[43] M. Caffo, H. Czyż and E. Remiddi, Nucl. Phys. B634 (2002) 309

[44] N.E. Ligterink, Phys. Rev. D61 (2000) 105010;

B. Kastening and H. Kleinert, Phys. Lett. A269 (2000) 50

[45] J. Fleischer and M.Y. Kalmykov, Phys. Lett. B470 (1999) 168

[46] G. Passarino, Nucl. Phys. B619 (2001) 257

[47] S. Groote, J.G. Körner and A.A. Pivovarov, Phys. Rev. D60 (1999) 061701

[48] S. Laporta, Int. J. Mod. Phys. A15 (2000) 5087

[49] A.T. Suzuki and A.G. Schmidt, J. Comput. Phys. 168 (2001) 207

[50] A.I. Davydychev and M.Y. Kalmykov, Nucl. Phys. B605 (2001) 266

[51] A. Aste and D. Trautmann, "Finite calculation of divergent selfenergy diagrams", Can. J. Phys. 81 (2003) 1433

[52] S. Bauberger, F.A. Berends, M. Böhm and M. Buza, Nucl. Phys. B434 (1995) 383

[53] D.I. Kazakov and A.V. Kotikov, Theor. Math. Phys. 73 (1988) 1264 [Teor. Mat. Fiz. 73 (1987) 348]

[54] A.A. Ovchinnikov, A.A. Pivovarov and L.R. Surguladze, Int. J. Mod. Phys. A6 (1991) 2025

[55] A.A. Pivovarov and L.R. Surguladze, Nucl. Phys. B360 (1991) 97, Yad. Fiz. 48 (1988) 1856 [Sov. J. Nucl. Phys. 48 (1989) 1117]

[56] S. Groote, J.G. Körner and A.A. Pivovarov, Phys. Rev. D61 (2000) 071501 
[57] B.L. Ioffe, Nucl. Phys. B188 (1981) 317; B191 (1981) 591(E)

[58] S. Groote, J.G. Körner and A.A. Pivovarov, "Analytical calculation of heavy baryon correlators in NLO of perturbative QCD", Published in Batavia 2000, Advanced computing and analysis techniques in physics research, p. 277 arXiv:hep-ph/0009218

[59] A.G. Grozin and O.I. Yakovlev, Phys. Lett. B285 (1992) 254

[60] R.J. Furnstahl, X.m. Jin and D.B. Leinweber, Phys. Lett. B387 (1996) 253

[61] X.m. Jin and J. Tang, Phys. Rev. D56 (1997) 515

[62] N.V. Krasnikov, A.A. Pivovarov and A.N. Tavkhelidze, JETP Lett. 36 (1982) 333 [Pisma Zh. Eksp. Teor. Fiz. 36 (1982) 272], Z. Phys. C19 (1983) 301

[63] A.A. Ovchinnikov, A.A. Pivovarov and L.R. Surguladze, Sov. J. Nucl. Phys. 48 (1988) 358 [Yad. Fiz. 48 (1988) 562]

[64] S. Groote and A.A. Pivovarov, Eur. Phys. J. C21 (2001) 133; JETP Lett. 75 (2002) 221 [Pisma Zh. Eksp. Teor. Fiz. 75 (2002) 267]

[65] A.L. Kataev, N.V. Krasnikov and A. A. Pivovarov, Nucl. Phys. B198 (1982) 508; B490 (1997) 505(E); Phys. Lett. B107 (1981) 115; A.A. Pivovarov, Phys. Atom. Nucl. 63 (2000) 1646 [Yad. Fiz. 63 N9 (2000) 1734]

[66] A. Bashir, R. Delbourgo and M.L. Roberts, J. Math. Phys. 42 (2001) 5553

[67] D.Y. Bardin and G. Passarino, "The standard model in the making: Precision study of the electroweak interactions", Oxford, UK: Clarendon, International series of monographs on physics, Vol. 104 (1999)

[68] D. Bardin, G. Passarino, L. Kalinovskaya, P. Christova, A. Andonov, S. Bondarenko and G. Nanava, "Project 'CalcPHEP': Calculus for precision high energy physics", arXiv:hep-ph/0202004

[69] I. Wetzorke and F. Karsch, Nucl. Phys. Proc. Suppl. 119 (2003) 278

[70] T. Doi, N. Ishi, M. Oka and H. Suganuma, "The lattice QCD simulation of the quark gluon mixed condensate $g$ at finite temperature and the phase transition of QCD", talk given at the 22nd International Symposium on Lattice Field Theory (Lattice 2004), Batavia, Illinois, 21-26 June 2004 arXiv:hep-lat/0409077 
[71] S. Narison and A.A. Pivovarov, Phys. Lett. B327 (1994) 341

[72] S. Weinberg, Physica A96 (1979) 327

[73] S.A. Larin, V.A. Matveev, A.A. Ovchinnikov and A.A. Pivovarov, Yad. Fiz. 44 (1986) 1066 [Sov. J. Nucl. Phys. 44 (1986) 690];

[74] T. Sakai, K. Shimizu and K. Yazaki, Prog. Theor. Phys. Suppl. 137 (2000) 121

[75] R.L. Jaffe and F. Wilczek, Phys. Rev. Lett. 91 (2003) 232003

[76] S.L. Zhu, Phys. Rev. Lett. 91 (2003) 232002

[77] I.I. Balitsky, D.I. D’Yakonov and A.V. Yung, Phys. Lett. B112 (1982) 71

[78] K.G. Chetyrkin and S. Narison, Phys. Lett. B485 (2000) 145

[79] R. Jaffe and F. Wilczek, Phys. World 17 (2004) 25

[80] J. Sugiyama, T. Doi and M. Oka, Phys. Lett. B581 (2004) 167

[81] N. Ishii, T. Doi, H. Iida, M. Oka, F. Okiharu and H. Suganuma, Phys. Rev. D71 (2005) 034001

[82] J.S. Schwinger, Phys. Rev. 82 (1951) 664

[83] A.A. Pivovarov, A.N. Tavkhelidze and V.F. Tokarev, Teor. Mat. Fiz. 60 (1984) 199 [Theor. Math. Phys. 60 (1985) 765]

[84] E. Witten, Nucl. Phys. B160 (1979) 57

[85] G. 't Hooft, Nucl. Phys. B72 (1974) 461

[86] S.R. Coleman and E. Weinberg, Phys. Rev. D7 (1973) 1888

[87] R. Jackiw and S. Templeton, Phys. Rev. D23 (1981) 2291

[88] R. Jackiw, Phys. Rev. D9 (1974) 1686

[89] J.M. Chung and B.K. Chung, J. Korean Phys. Soc. 39 (2001) 971

[90] J.M. Chung and B.K. Chung, Phys. Rev. D59 (1999) 105014 
[91] K. Kajantie, M. Laine, K. Rummukainen and Y. Schröder, JHEP 0304 (2003) 036

[92] T. Hatsuda, Nucl. Phys. A544 (1992) 27

[93] D.J. Gross, R.D. Pisarski and L.G. Yaffe, Rev. Mod. Phys. 53 (1981) 43

[94] T. Nishikawa, O. Morimatsu and Y. Hidaka, Phys. Rev. D68 (2003) 076002

[95] J.O. Andersen, E. Braaten and M. Strickland, Phys. Rev. D62 (2000) 045004

[96] T. Appelquist and R.D. Pisarski, Phys. Rev. D23 (1981) 2305

[97] T. Doi, N. Ishii, M. Oka and H. Suganuma, Phys. Rev. D70 (2004) 034510

[98] J.F. Yang, J. Zhou and C. Wu, Commun. Theor. Phys. 40 (2003) 461

[99] H. Van Hees and J. Knoll, Phys. Rev. D65 (2002) 105005

[100] N.P. Mehta, C. Felline, J.R. Shepard and J. Piekarewicz, Phys. Rev. C68 (2003) 034003

[101] S.A. Kulagin and A.A. Pivovarov, Yad. Fiz. 45 (1987) 952

[102] U.G. Meißner, Nucl. Phys. A751 (2005) 149

[103] J. Bijnens, U.G. Meißner and A. Wirzba, "Effective field theories in nuclear, particle and atomic physics", to appear in the proceedings of 337th International We-Heraeus Seminar (EFT 04): Effective Field Theories in Nuclear, Particle and Atomic Physics, Bad Honnef, Germany, 13-17 December 2004 arXiv:hep-ph/0502008

[104] E. Mirkes, "Theory of jets in deep elastic scattering", Report No. TTP-97-39 hep-ph/9711224

[105] L. Platter, H.W. Hammer and U.G. Meißner, Nucl. Phys. A714 (2003) 250

[106] A.A. Pivovarov, Int. J. Mod. Phys. A10 (1995) 3125

[107] J. Gasser, M.A. Ivanov, E. Lipartia, M. Mojzis and A. Rusetsky, Eur. Phys. J. C26 (2002) 13

[108] L.V. Avdeev, Comput. Phys. Commun. 98 (1996) 15

[109] C. Itzykson and J.B. Zuber, "Quantum Field Theory", McGraw-Hill, New York, 1980 
[110] D.J. Broadhurst, Eur. Phys. J. C8 (1999) 311

[111] K.G. Chetyrkin, A.L. Kataev and F.V. Tkachov, Nucl. Phys. B174 (1980) 345

[112] A.E. Terrano, Phys. Lett. B93 (1980) 424

[113] N.N. Bogoliubov and O.S. Parasiuk, Acta Math. 97 (1957) 227

[114] N.N. Bogoliubov and D.V. Shirkov, "Quantum fields", Benjamin, 1983

[115] L.N. Lipatov, Nucl. Phys. B452 (1995) 369

[116] L.N. Lipatov, "The creation of quantum chromodynamics and the effective energy", Bologna, Italy: Univ. Bologna (1998)

[117] L.N. Lipatov, Nucl. Phys. Proc. Suppl. 64 (1998) 188

[118] N.V. Krasnikov and A.B. Kyatkin, Mod. Phys. Lett. A6 (1991) 1315

[119] G. ’t Hooft and M.J.G. Veltman, Nucl. Phys. B44 (1972) 189

[120] G. 't Hooft, Nucl. Phys. B62 (1973) 444

[121] G. 't Hooft, Nucl. Phys. B61 (1973) 455

[122] K.G. Chetyrkin, J.H. Kühn and A. Kwiatkowski, Phys. Rept. 277 (1996) 189

[123] K. Hepp, Commun. Math. Phys. 2 (1966) 301

[124] J. Lowenstein, M. Weinstein and W. Zimmermann, Phys. Rev. D10 (1974) 1854

[125] S.A. Anikin, M.K. Polivanov and O.I. Zavyalov, Theor. Math. Phys. 17 (1973) 1082

[126] A.A. Pivovarov, N.N. Tavkhelidze and V.F. Tokarev, Phys. Lett. B132 (1983) 402

[127] K.G. Chetyrkin and A.A. Pivovarov, Nuovo Cim. A100 (1988) 899

[128] A.A. Pivovarov and V.F. Tokarev, Yad. Fiz. 41 (1985) 524

[129] S. Weinberg, "The Quantum Theory of Fields", Vol. II, Cambridge University Press, 1996

[130] C.S. Meijer, Proc. Amsterdam Akad. Wet. (1940) 599; 702

[131] A. Erdelyi (Ed.), "Tables of integral transformations", Volume 2, Bateman manuscript project, 1954 
[132] A.A. Pivovarov, Phys. Lett. B236 (1990) 214; Phys. Lett. B263 (1991) 282

[133] G.N. Watson, "Theory of Bessel functions", Cambridge, 1944

[134] A.P. Prudnikov, Yu.A. Brychkov and O.I. Marichev, "Integrals and Series", Vol. 2, Gordon and Breach, New York, 1990

[135] I.S. Gradshteyn and I.M. Ryzhik, "Tables of integrals, series, and products", Academic Press, 1994

[136] D.J. Broadhurst, Z. Phys. C54 (1992) 599

[137] S. Laporta, Phys. Lett. B549 (2002) 115

[138] H.S. Do, Ph. D. thesis, University of Mainz, Germany 2003

[139] O.M. Ogreid and P. Osland, J. Comput. Appl. Math. 136 (2001) 389

[140] D.J. Broadhurst and D. Kreimer, Int. J. Mod. Phys. C6 (1995) 519; Phys. Lett. B393 (1997) 403

[141] S. Groote, J.G. Körner and A.A. Pivovarov, Eur. Phys. J. C36 (2004) 471

[142] S. Eidelman et al. [Particle Data Group], Phys. Lett. B592 (2004) 1

[143] R.E. Cutkosky, J. Math. Phys. 1 (1960) 429

[144] Y. Zhou, "Imaginary part of Feynman amplitude, cutting rules and optical theorem" arXiv:hep-ph/0412204

[145] N. Isgur and M.B. Wise, Phys. Lett. B232 (1989) 113; Phys. Lett. B237 (1990) 527; F. Hussain, J.G. Körner, K. Schilcher, G. Thompson and Y.L. Wu, Phys. Lett. B249 (1990) 295; J.G. Körner and G. Thompson, Phys. Lett. B264 (1991) 185; S. Balk, J.G. Körner and D. Pirjol, Nucl. Phys. B428 (1994) 499; D.J. Broadhurst and A.G. Grozin, Phys. Rev. D52 (1995) 4082

[146] K.G. Chetyrkin, J.H. Kühn and A.A. Pivovarov, Nucl. Phys. B533 (1998) 473

[147] J.G. Körner, F. Krajewski and A.A. Pivovarov, Eur. Phys. J. C12 (2000) 461; C14 (2000) 123; C20 (2001) 259

[148] D.S. Gorbunov and A.A. Pivovarov, Phys. Rev. D71 (2005) 013002 
[149] K.G. Chetyrkin and F.V. Tkachov, Phys. Lett. B114 (1982) 340

[150] F.V. Tkachov, Phys. Lett. B125 (1983) 85

[151] V.M. Braun and Y.M. Shabelski, Sov. J. Nucl. Phys. 50 (1989) 306

[152] A. Pich and E. De Rafael, Phys. Lett. B158 (1985) 477

[153] K.G. Chetyrkin, A.L. Kataev, A.B. Krasulin and A.A. Pivovarov, Phys. Lett. B174 (1986) 104

[154] K.G. Chetyrkin, J.H. Kühn and M. Steinhauser, Phys. Lett. B371 (1996) 93

[155] K.G. Chetyrkin, J.H. Kühn and M. Steinhauser, Nucl. Phys. B505 (1997) 40

[156] J. Fleischer and O.V. Tarasov, Nucl. Phys. Proc. Suppl. 37B (1994) 115

[157] E. Bagan, H.G. Dosch, P. Gosdzinsky, S. Narison and J.M. Richard, Z. Phys. C64 (1994) 57

[158] J.H. Kühn, A.A. Penin and A.A. Pivovarov, Nucl. Phys. B534 (1998) 356

[159] A.A. Penin and A.A. Pivovarov, Phys. Lett. B435 (1998) 413; B443 (1998) 264; Nucl. Phys. B550 (1999) 375; B549 (1999) 217; Phys. Atom. Nucl. 64 (2001) 275 [Yad. Fiz. 64 (2001) 323];

[160] K. Melnikov and A. Yelkhovsky, Nucl. Phys. B528 (1998) 59

[161] M. Beneke, A. Signer and V.A. Smirnov, Phys. Rev. Lett. 80 (1998) 2535

[162] A. Czarnecki and K. Melnikov, Phys. Rev. Lett. 80 (1998) 2531

[163] A.H. Hoang et al., Eur. Phys. J. direct C2 (2000) 3

[164] A.H. Hoang and T. Teubner, Phys. Rev. D60 (1999) 114027

[165] T. Nagano, A. Ota and Y. Sumino, Phys. Rev. D60 (1999) 114014

[166] A.A. Pivovarov, Phys. Lett. B475 (2000) 135; A.A. Pivovarov, "Heavy quark production near the threshold in QCD", talk given at 10th Lomonosov conference on elementary paricle physics, Moscow, Russia, 23-29 August 2001 arXiv:hep-ph/0110398 
[167] V.A. Smirnov, Phys. Lett. B404 (1997) 101; M. Beneke and V.A. Smirnov, Nucl. Phys. B522 (1998) 321; V.A. Smirnov and E.R. Rakhmetov, Theor. Math. Phys. 120 (1999) 870 [Teor. Mat. Fiz. 120 (1999) 64]

[168] A.I. Davydychev and V.A. Smirnov, Nucl. Phys. B554 (1999) 391

[169] V.A. Smirnov, Phys. Lett. B465 (1999) 226

[170] M. Abramowitz, I.A. Stegun (eds.), "Handbook of Mathematical Functions", Dover Publ. Inc., New York, 9th Printing, 1970

[171] P.B. Gilkey, J. Diff. Geom. 10 (1975) 601

[172] A.M. Polyakov, Phys. Lett. B72 (1978) 477

[173] P. Mansfield, Nucl. Phys. B272 (1986) 439

[174] V.P. Gusynin, A.H. Hams and M. Reenders, Phys. Rev. D53 (1996) 2227

[175] J. Bartels, V.S. Fadin and L.N. Lipatov, Nucl. Phys. B698 (2004) 255

[176] S.V. Mikhailov and A.V. Radyushkin, Theor. Math. Phys. 65 (1986) 999 [Teor. Mat. Fiz. 65 (1985) 44]

[177] A.A. Slavnov, Nucl. Phys. Proc. Suppl. 88 (2000) 210

[178] E. Dudas, T. Gherghetta and S. Groot Nibbelink, Phys. Rev. D70 (2004) 086012

[179] M. Cvetič, H. Lü and C. N. Pope, Phys. Rev. D70 (2004) 081502

[180] M.W. Kalinowski, Int. J. Theor. Phys. 4 (1986) 327

[181] N.V. Krasnikov, JETP Lett. 51 (1990) 4 [Pisma Zh. Eksp. Teor. Fiz. 51 (1990) 5]; Phys. Lett. B273 (1991) 246

[182] J.G. Körner, A.I. Onishchenko, A.A. Petrov and A.A. Pivovarov, Phys. Rev. Lett. 91 (2003) 192002

[183] A.A. Pivovarov, "Sum rules for $B^{0}-\bar{B}^{0}$ mixing at NLO of pQCD", talk given at 11th International Conference in Quantum ChromoDynamics (QCD 04), Montpellier, France, 5-9 July 2004 arXiv:hep-ph/0410046. A.A. Pivovarov, "Computing non-factorizable pQCD corrections to hadronic $B^{0}-\bar{B}^{0}$ mixing matrix element within sum rules technique for 
three-point Green functions", talk given at 13th International Seminar on High-Energy Physics: Quarks 2004, Pushkinskie Gory, Russia, 24-30 May 2004 arXiv:hep-ph/0409257

[184] J.H. Kühn, A.I. Onishchenko, A.A. Pivovarov and O.L. Veretin, Phys. Rev. D68 (2003) 033018

[185] M. Steinhauser, Comput. Phys. Commun. 134 (2001) 335

[186] L. Avdeev, J. Fleischer, S. Mikhailov and O. Tarasov, Phys. Lett. B336 (1994) 560; B349 (1995) 597(E)

[187] K.G. Chetyrkin, J.H. Kühn and M. Steinhauser, Phys. Lett. B351 (1995) 331

[188] A.B. Krasulin, A.A. Ovchinnikov and A.A. Pivovarov, Sov. J. Nucl. Phys. 48 (1988) 655 [Yad. Fiz. 48, 1029 (1988)]

[189] A.A. Pivovarov, Nucl. Phys. B396 (1993) 119

[190] F.V. Tkachov, Phys. Lett. B100 (1981) 65

[191] K.G. Chetyrkin and F.V. Tkachov, Nucl. Phys. B192 (1981) 159

[192] P.A. Baikov, Phys. Lett. B385 (1996) 404

[193] D.J. Broadhurst, Z. Phys. C47 (1990) 115

[194] S.C. Generalis, J. Phys. G16 (1990) 785

[195] D. Kreimer, Phys. Lett. B273 (1991) 277

[196] L. Brücher, J. Franzkowski and D. Kreimer, "xloops: A program package calculating oneand two-loop Feynman diagrams", Report No. MZ-TH/97-35 arXiv:hep-ph/9710484 